\title{
SPARSE-GRID POLYNOMIAL INTERPOLATION APPROXIMATION AND INTEGRATION FOR PARAMETRIC AND STOCHASTIC ELLIPTIC PDES WITH LOGNORMAL INPUTS
}

\author{
DINH DŨNG
}

\begin{abstract}
By combining a certain approximation property in the spatial domain, and weighted $\ell_{2}$-summability of the Hermite polynomial expansion coefficients in the parametric domain obtained in Bachmayr et al. [ESAIM: M2AN 51 (2017) 341-363] and Bachmayr et al. [SIAM J. Numer. Anal. 55 (2017) 2151-2186], we investigate linear non-adaptive methods of fully discrete polynomial interpolation approximation as well as fully discrete weighted quadrature methods of integration for parametric and stochastic elliptic PDEs with lognormal inputs. We construct such methods and prove convergence rates of the approximations by them. The linear non-adaptive methods of fully discrete polynomial interpolation approximation are sparse-grid collocation methods which are certain sums taken over finite nested Smolyak-type indices sets of mixed tensor products of dyadic scale successive differences of spatial approximations of particular solvers, and of successive differences of their parametric Lagrange interpolating polynomials. The Smolyak-type sparse interpolation grids in the parametric domain are constructed from the roots of Hermite polynomials or their improved modifications. Moreover, they generate in a natural way fully discrete weighted quadrature formulas for integration of the solution to parametric and stochastic elliptic PDEs and its linear functionals, and the error of the corresponding integration can be estimated via the error in the Bochner space $L_{1}\left(\mathbb{R}^{\infty}, V, \gamma\right)$ norm of the generating methods where $\gamma$ is the Gaussian probability measure on $\mathbb{R}^{\infty}$ and $V$ is the energy space. We also briefly consider similar problems for parametric and stochastic elliptic PDEs with affine inputs, and problems of non-fully discrete polynomial interpolation approximation and integration. In particular, the convergence rates of non-fully discrete polynomial interpolation approximation and integration obtained in this paper significantly improve the known ones.
\end{abstract}

Mathematics Subject Classification. 65C30, 65D05, 65D32, 65N15, 65N30, 65N35.

Received March 27, 2020. Accepted April 11, 2021.

\section{INTRODUCTION}

One of basic problems in Uncertainty Quantification are approximation and numerical integration for parametric and stochastic PDEs. Since the number of parametric variables may be very large or even infinite, they

\footnotetext{
Keywords and phrases. High-dimensional approximation, parametric and stochastic elliptic PDEs, lognormal inputs, collocation approximation, fully discrete non-adaptive polynomial interpolation approximation, fully discrete non-adaptive integration.

Information Technology Institute, Vietnam National University, 144 Xuan Thuy, Cau Giay, Hanoi, Vietnam.

*Corresponding author: dinhzung@gmail.com
} 
are treated as high-dimensional or infinite-dimensional approximation problems. Let $D \subset \mathbb{R}^{d}$ be a bounded Lipschitz domain. Consider the diffusion elliptic equation

$$
-\operatorname{div}(a \nabla u)=f \quad \text { in } \quad D,\left.\quad u\right|_{\partial D}=0,
$$

for a given fixed right-hand side $f$ and spatially variable scalar diffusion coefficient $a$. Denote by $V:=H_{0}^{1}(D)$ the energy space and let $V^{\prime}=H^{-1}(D)$ be the conjungate space of $V$. If $a$ satisfies the ellipticity assumption

$$
0<a_{\min } \leq a \leq a_{\max }<\infty,
$$

by the well-known Lax-Milgram lemma, for any $f \in V^{\prime}$, there exists a unique solution $u \in V$ in weak form which satisfies the variational equation

$$
\int_{D} a \nabla u \cdot \nabla v \mathrm{~d} \boldsymbol{x}=\langle f, v\rangle, \quad \forall v \in V
$$

We consider diffusion coefficients having a parametrized form $a=a(\boldsymbol{y})$, where $\boldsymbol{y}=\left(y_{j}\right)_{j \in \mathbb{N}}$ is a sequence of real-valued parameters ranging in the set $\mathbb{U}^{\infty}$ which is either $\mathbb{R}^{\infty}$ or $\mathbb{I}^{\infty}:=[-1,1]^{\infty}$. In this case, the solution $u(\boldsymbol{y})$ to the parametrized diffusion elliptic equation

$$
-\operatorname{div}(a(\boldsymbol{y}) \nabla u(\boldsymbol{y}))=f \quad \text { in } \quad D,\left.\quad u(\boldsymbol{y})\right|_{\partial D}=0,
$$

can be considered as a map $\boldsymbol{y} \mapsto u(\boldsymbol{y})$ from $\mathbb{U}^{\infty}$ to the space $V$. The objective is to achieve numerical approximation of this complex map by a small number of parameters with some guaranteed error in a given norm. Depending on the nature of the modeled object, the parameter $\boldsymbol{y}$ may be either deterministic or random. In the present paper, we consider the so-called lognormal case when $\mathbb{U}^{\infty}=\mathbb{R}^{\infty}$ and the diffusion coefficient $a$ is of the form

$$
a(\boldsymbol{y})=\exp (b(\boldsymbol{y})), \quad b(\boldsymbol{y})=\sum_{j=1}^{\infty} y_{j} \psi_{j},
$$

where the $y_{j}$ are i.i.d. standard Gaussian random variables and $\psi_{j} \in L_{\infty}(D)$. We also briefly consider the affine case when $\mathbb{U}^{\infty}=\mathbb{I}^{\infty}$ and the diffusion coefficient $a$ is of the form

$$
a(\boldsymbol{y})=\bar{a}+\sum_{j=1}^{\infty} y_{j} \psi_{j} .
$$

In order to study fully discrete approximations of the solution $u(\boldsymbol{y})$ to the parametrized elliptic PDEs (1.1), we assume that $f \in L_{2}(D)$ and $a(\boldsymbol{y}) \in W_{\infty}^{1}(D)$, and hence we obtain that $u(\boldsymbol{y})$ has the second higher regularity, i.e., $u(\boldsymbol{y}) \in W$ where $W$ is the space

$$
W:=\left\{v \in V: \Delta v \in L^{2}(D)\right\}
$$

equipped with the norm

$$
\|v\|_{W}:=\|\Delta v\|_{L^{2}(D)},
$$

which coincides with the Sobolev space $V \cap H^{2}(D)$ with equivalent norms if the domain $D$ has $C^{1,1}$ smoothness, see Theorem 2.5.1.1 of [19]. Moreover, we assume that there holds the following approximation property for the spaces $V$ and $W$.

Assumption 1.1. There are a sequence $\left(V_{n}\right)_{n \in \mathbb{N}_{0}}$ of subspaces $V_{n} \subset V$ of dimension $\leq m$, and a sequence $\left(P_{n}\right)_{n \in \mathbb{N}_{0}}$ of linear operators from $V$ into $V_{n}$, and a number $\alpha>0$ such that

$$
\left\|P_{n}(v)\right\|_{V} \leq C\|v\|_{V}, \quad\left\|v-P_{n}(v)\right\|_{V} \leq C n^{-\alpha}\|v\|_{W}, \quad \forall n \in \mathbb{N}_{0}, \quad \forall v \in W .
$$


A basic role in the approximation and numerical integration for parametric and stochastic PDEs are generalized polynomial chaos (gpc) expansions for the dependence on the parametric variables. We refer the reader to $[9,12,20,28,29]$ and references there for different aspects in approximation for parametric and stochastic PDEs. In [5-11], based on the conditions $\left(\left\|\psi_{j}\right\|_{W_{\infty}^{1}(D)}\right)_{j \in \mathbb{N}} \in \ell_{p}(\mathbb{N})$ for some $0<p<1$ on the affine expansion (1.4), the authors have proven the $\ell_{p}$-summability of the coefficients in a Taylor or Legendre polynomials expansion and hence proposed best adaptive $n$-term methods of Galerkin and collocation approximations in energy norm by choosing the set of the $n$ most useful terms in these expansions. To derive a fully discrete approximation the best $n$-term approximants are then approximated by finite element methods. Similar results have been received in [24] for Galerkin approximation in the lognormal case based on the conditions $\left(j\left\|\psi_{j}\right\|_{W_{\infty}^{1}(D)}\right)_{j \in \mathbb{N}} \in \ell_{p}(\mathbb{N})$ for some $0<p<1$. In these papers, they did not take into account support properties of the functions $\psi_{j}$.

A different approach to studying summability that takes into account the support properties has been recently proposed in [2] for the affine case and [3] for the lognormal case. This approach leads to significant improvements on the results on $\ell_{p}$-summability when the functions $\psi_{j}$ have limited overlap, such as splines, finite elements or wavelet bases. These results by themselves do not imply practical applications, because they do not cover the approximation of the expansion coefficients which are functions of the spatial variable.

In the recent paper [1], the rates of fully discrete adaptive best $n$-term Taylor, Jacobi and Hermite polynomial approximations for elliptic PDEs with affine or lognormal parametrizations of the diffusion coefficients have been obtained based on combining a certain approximation property on the spatial domain, and extensions of the results on $\ell_{p}$-summability of $[2,3]$ to higher-order Sobolev norms of corresponding Taylor, Jacobi and Hermite expansion coefficients. These results providing a benchmark for convergence rates, are not constructive. In the case when $\ell_{p}$-summable sequences of Sobolev norms of expansion coefficients have an $\ell_{p}$-summable majorant sequence, these convergence rates can be achieved by linear methods of gpc expansion and collocation approximations in the affine case $[9,14-16,32,34]$. However, this non-adaptive approach is not applicable for the improvement of $\ell_{p}$-summability in [1-3] since the weakened $\ell_{p}$-assumption leads only to the $\ell_{p}$-summability of expansion coefficients, but not to an $\ell_{p}$-summable majorant sequence. Non-adaptive non-fully discrete methods have been considered in [18] for polynomial collocation approximation, and in [4] for weighted integration (see also Rem. 3.2 of [2] and Rem. 5.1 of [3] for briefly considering non-adaptive non-fully discrete approximations by truncated gpc expansions).

Let us briefly describe the main contribution of the present paper. By combining spatial and parametric approximability, namely, the approximation property in Assumption 1.1 in the spatial domain and weighted $\ell_{2}$-summability of the $V$ and $W$ norms of Hermite polynomial expansion coefficients obtained in [1,3], we investigate linear non-adaptive methods of fully discrete approximation by truncated Hermite gpc expansion and polynomial interpolation approximation as well as fully discrete weighted quadrature methods of integration for parametric and stochastic elliptic PDEs with lognormal inputs (1.3). We construct such methods and prove convergence rates of the approximations by them. We show that the convergence rate in terms of the dimension of the approximation space of adaptive fully discrete approximation by truncated Hermite gpc expansion obtained in [1], is achieved by linear non-adaptive methods of fully discrete approximation by truncated Hermite gpc expansion approximation. The linear non-adaptive methods of fully discrete polynomial interpolation approximation are sparse-grid collocation methods which are certain sums taken over finite nested Smolyaktype indices sets of tensor products of dyadic scale successive differences of spatial approximations of particular solvers, and of successive differences of their parametric Lagrange interpolating polynomials. The Smolyak-type sparse interpolation grids in the parametric domain are constructed from the roots of Hermite polynomials or their improved modifications. Moreover, these methods generate in a natural way fully discrete weighted quadrature formulas for integration of the solution $u(\boldsymbol{y})$ and its linear functionals, and the error of the corresponding integration can be estimated via the error in the space $L_{1}\left(\mathbb{R}^{\infty}, V, \gamma\right)$ norm of the generating methods where $\gamma$ is the Gaussian probability measure on $\mathbb{R}^{\infty}$. The convergence rate of fully discrete integration is better than the convergence rate of the generating fully discrete polynomial interpolation approximation due to the simple but useful observation that the integral $\int_{\mathbb{R}} v(y) \mathrm{d} \gamma(y)$ is zero if $v(y)$ is an odd function and $\gamma$ is the Gaussian probability measure on $\mathbb{R}$. (This property has been used in [32-34] for improving convergence rate of integration 
in the affine case.) We also briefly consider similar problems for parametric and stochastic elliptic PDEs with affine inputs (1.4) by using counterparts-results in [1,2], and problems of non-fully discrete polynomial interpolation approximation and integration similar to those treated in $[4,18]$. In particular, the convergence rates of non-fully discrete interpolation approximation and integration in terms of number of the evaluation points obtained in this paper, are significantly better than those which have been proven in $[4,18]$.

Finally, let us notice that the aim of this paper is to establish approximation results which should show possibilities of non-adaptive approximation methods and convergence rates of approximation by such methods for the parametrized diffusion elliptic equation (1.2) with lognormal inputs. The two most popular numerical methods are Galerkin projection and collocation. Since in the lognormal case, the diffusion coefficient $a(\boldsymbol{y})(\boldsymbol{x})$ is not uniformly bounded in $\boldsymbol{y} \in \mathbb{R}^{\infty}$, there is no a well-posed linear variational problem on the space $L_{2}\left(\mathbb{R}^{\infty}, V, \gamma\right)$ for Galerkin approximation. Some best $n$-term Galerkin approximations with respect to a "stronger" Gaussian measure $\gamma_{\varrho}$ were considered in [24]. Collocation methods will be discussed in a forthcoming paper which extends the results in the affine case $[33,34]$.

The paper is organized as follows. In Sections 2-4, we construct general linear fully discrete and non-fully discrete methods of Hermite gpc expansion and polynomial interpolation approximations in the Bochner space $L_{p}\left(\mathbb{R}^{\infty}, X^{1}, \gamma\right)$, and quadrature of functions taking values in $X^{2}$ and having a weighted $\ell_{2}$-summability of Hermite expansion coefficients for Hilbert spaces $X^{1}$ and $X^{2}$ satisfying a certain "spatial" approximation property (see (2.3)). In particular, in Section 2, we prove convergence rates of general linear fully discrete methods of approximation approximation by truncated Hermite gpc expansion; in Section 3, we prove convergence rates of general linear fully discrete and non-fully discrete polynomial interpolation methods of approximation; in Section 4, we prove convergence rates of general linear fully discrete and non-fully discrete quadrature for integration. In Section 5, we apply the results of Sections 2-4 to obtain the main results of this paper on convergence rates of linear non-adaptive methods of fully discrete approximation by truncated Hermite gpc expansion, and fully discrete and non-fully discrete polynomial interpolation approximation and weighted quadrature methods of integration for parametric and stochastic elliptic PDEs with lognormal inputs. In Section 6, by extending the theory in Sections 2-4, we briefly consider similar problems for parametric and stochastic elliptic PDEs with affine inputs.

\section{LineAR APPROXimation By tRUnCATED HeRmite GPC EXPANSiON}

In this section, we treat a general linear fully discrete approximation by truncated Hermite gpc series of functions from the Bochner space $L_{2}\left(\mathbb{R}^{\infty}, X^{2}, \gamma\right)$. The approximation error is measured in the Bochner space $L_{p}\left(\mathbb{R}^{\infty}, X^{1}, \gamma\right)$ with $0<p \leq 2$. Here, $X^{1}$ and $X^{2}$ are Hilbert spaces, and $\gamma$ is the infinite tensor product Gaussian probability measure. We construct linear (non-adaptive) methods of this approximation and prove convergence rates for the approximation error.

We first recall a concept of infinite tensor product of probability measures. (For details see, e.g., [23], pp. 429435.) Let $\mu(y)$ be a probability measure on $\mathbb{U}$, where $\mathbb{U}$ is either $\mathbb{R}$ or $\mathbb{I}:=[-1,1]$. We introduce the probability measure $\mu(\boldsymbol{y})$ on $\mathbb{U}^{\infty}$ as the infinite tensor product of probability measures $\mu\left(y_{i}\right)$ :

$$
\mu(\boldsymbol{y}):=\bigotimes_{j \in \mathbb{N}} \mu\left(y_{j}\right), \quad \boldsymbol{y}=\left(y_{j}\right)_{j \in \mathbb{N}} \in \mathbb{U}^{\infty} .
$$

The sigma algebra for $\mu(\boldsymbol{y})$ is generated by the set of cylinders $A:=\prod_{j \in \mathbb{N}} A_{j}$, where $A_{j} \subset \mathbb{U}$ are univariate $\mu$-measurable sets and only a finite number of $A_{i}$ are different from $\mathbb{U}$. For such a set $A$, we have $\mu(A)=$ $\prod_{j \in \mathbb{N}} \mu\left(A_{j}\right)$. If $\varrho(y)$ is the density of $\mu(y)$, i.e., $\mathrm{d} \mu(y)=\varrho(y) \mathrm{d} y$, then we write

$$
\mathrm{d} \mu(\boldsymbol{y}):=\bigotimes_{j \in \mathbb{N}} \varrho\left(y_{j}\right) \mathrm{d}\left(y_{j}\right), \quad \boldsymbol{y}=\left(y_{j}\right)_{j \in \mathbb{N}} \in \mathbb{U}^{\infty} .
$$

Let $X$ be a Hilbert space and $0<p<\infty$. The probability measure $\mu(\boldsymbol{y})$ induces the Bochner space $L_{p}\left(\mathbb{U}^{\infty}, X, \mu\right)$ of strongly $\mu$-measurable mappings $v$ from $\mathbb{U}^{\infty}$ to $X$ which are $p$-summable. The (quasi-)norm in 
$L_{p}\left(\mathbb{U}^{\infty}, X, \mu\right)$ is defined by

$$
\|v\|_{L_{p}\left(\mathbb{U}^{\infty}, X, \mu\right)}:=\left(\int_{\mathbb{U}^{\infty}}\|v(\cdot, \boldsymbol{y})\|_{X}^{p} \mathrm{~d} \mu(\boldsymbol{y})\right)^{1 / p} .
$$

Notice that if $X$ is separable, $L_{2}\left(\mathbb{U}^{\infty}, X, \mu\right)$ is the tensor product of the Hilbert spaces $L_{2}\left(\mathbb{U}^{\infty}, \mathbb{R}, \mu\right)$ and $X$.

In the present paper, we focus our attention mainly to the lognormal case with $\mathbb{U}^{\infty}=\mathbb{R}^{\infty}$ and $\mu(\boldsymbol{y})=\gamma(\boldsymbol{y})$, the infinite tensor product Gaussian probability measure. Let $\gamma(y)$ be the probability measure on $\mathbb{R}$ with the standard Gaussian density:

$$
\mathrm{d} \gamma(y):=g(y) \mathrm{d} y, \quad g(y):=\frac{1}{\sqrt{2 \pi}} e^{-y^{2} / 2} .
$$

Then the infinite tensor product Gaussian probability measure $\gamma(\boldsymbol{y})$ on $\mathbb{R}^{\infty}$ can be defined by

$$
\mathrm{d} \gamma(\boldsymbol{y}):=\bigotimes_{j \in \mathbb{N}} g\left(y_{j}\right) \mathrm{d}\left(y_{j}\right), \quad \boldsymbol{y}=\left(y_{j}\right)_{j \in \mathbb{N}} \in \mathbb{R}^{\infty} .
$$

A powerful strategy for the approximation of functions $v$ in $L_{2}\left(\mathbb{R}^{\infty}, X, \gamma\right)$ is based on the truncation of the Hermite gpc expansion

$$
v(\boldsymbol{y})=\sum_{\boldsymbol{s} \in \mathbb{F}} v_{\boldsymbol{s}} H_{\boldsymbol{s}}(\boldsymbol{y}), \quad v_{\boldsymbol{s}} \in X .
$$

Here $\mathbb{F}$ is the set of all sequences of non-negative integers $s=\left(s_{j}\right)_{j \in \mathbb{N}}$ such that their support $\operatorname{supp}(\boldsymbol{s}):=\{j \in$ $\left.\mathbb{N}: s_{j}>0\right\}$ is a finite set, and

$$
H_{\boldsymbol{s}}(\boldsymbol{y})=\bigotimes_{j \in \mathbb{N}} H_{s_{j}}\left(y_{j}\right), \quad v_{\boldsymbol{s}}:=\int_{\mathbb{R}^{\infty}} v(\boldsymbol{y}) H_{\boldsymbol{s}}(\boldsymbol{y}) \mathrm{d} \gamma(\boldsymbol{y}), \quad \boldsymbol{s} \in \mathbb{F},
$$

with $\left(H_{k}\right)_{k \geq 0}$ being the Hermite polynomials normalized according to $\int_{\mathbb{R}}\left|H_{k}(y)\right|^{2} g(y) \mathrm{d} y=1$. It is well-known that $\left(H_{\boldsymbol{s}}\right)_{\boldsymbol{s} \in \mathbb{F}}$ is an orthonormal basis of $L_{2}\left(\mathbb{R}^{\infty}, \mathbb{R}, \gamma\right)$. Moreover, for every $v \in L_{2}\left(\mathbb{R}^{\infty}, X, \gamma\right)$ represented by the series (2.2) it holds Parseval's identity

$$
\|v\|_{L_{2}\left(\mathbb{R}^{\infty}, X, \gamma\right)}^{2}=\sum_{\boldsymbol{s} \in \mathbb{F}}\left\|v_{\boldsymbol{s}}\right\|_{X}^{2} .
$$

We make use of the abbreviations: $L_{p}\left(\mathbb{R}^{\infty}, \mu\right):=L_{p}\left(\mathbb{R}^{\infty}, \mathbb{R}, \mu\right) ; \mathcal{L}_{p}(X):=L_{p}\left(\mathbb{R}^{\infty}, X, \gamma\right)$ for $0<p<\infty$. We use letter $C$ to denote a general positive constant which may take different values, and $C_{p, q, \alpha, D, \ldots}$ a constant depending on $p, q, \alpha, D, \ldots$

To construct general linear fully discrete methods of approximation in the Bochner space $\mathcal{L}_{p}\left(X^{1}\right)$ and of integration of functions taking values in $X^{2}$, we need the following assumption on approximation property for $X^{1}$ and $X^{2}$, which is a generalization of Assumption 1.1.

Assumption 2.1. The Hilbert space $X^{2}$ is a linear subspace of the Hilbert space $X^{1}$ and that $\|\cdot\|_{X^{1}} \leq C\|\cdot\|_{X^{2}}$. There are a sequence $\left(V_{n}\right)_{n \in \mathbb{N}_{0}}$ of subspaces $V_{n} \subset X^{1}$ of dimension $\leq n$, and a sequence $\left(P_{n}\right)_{n \in \mathbb{N}_{0}}$ of linear operators from $X^{1}$ into $V_{n}$, and a number $\alpha>0$ such that

$$
\left\|P_{n}(v)\right\|_{X^{1}} \leq C\|v\|_{X^{1}}, \quad\left\|v-P_{n}(v)\right\|_{X^{1}} \leq C n^{-\alpha}\|v\|_{X^{2}}, \quad \forall n \in \mathbb{N}_{0}, \quad \forall v \in X^{2} .
$$

For $k \in \mathbb{N}_{0}$, we define

$$
\delta_{k}(v):=P_{2^{k}}(v)-P_{2^{k-1}}(v), k \in \mathbb{N}, \quad \delta_{0}(v):=P_{0}(v) .
$$

We can represent every $v \in X^{2}$ by the series

$$
v=\sum_{k=0}^{\infty} \delta_{k}(v)
$$


converging in $X^{1}$ and satisfying the estimate

$$
\left\|\delta_{k}(v)\right\|_{X^{1}} \leq C 2^{-\alpha k}\|v\|_{X^{2}}, \quad k \in \mathbb{N}_{0}
$$

For a finite subset $G$ in $\mathbb{N}_{0} \times \mathbb{F}$, denote by $\mathcal{V}(G)$ the subspace in $\mathcal{L}_{2}\left(X^{1}\right)$ of all functions $v$ of the form

$$
v=\sum_{(k, s) \in G} v_{k} H_{s}, \quad v_{k} \in V_{2^{k}}
$$

Let Assumption 2.1 hold for Hilbert spaces $X^{1}$ and $X^{2}$. We define the linear operator $\mathcal{S}_{G}: \mathcal{L}_{2}\left(X^{2}\right) \rightarrow \mathcal{V}(G)$ by

$$
\mathcal{S}_{G} v:=\sum_{(k, \boldsymbol{s}) \in G} \delta_{k}\left(v_{\boldsymbol{s}}\right) H_{\boldsymbol{s}}
$$

for $v \in \mathcal{L}_{2}\left(X^{2}\right)$ represented by the series

$$
v=\sum_{\boldsymbol{s} \in \mathbb{F}} v_{\boldsymbol{s}} H_{\boldsymbol{s}}, \quad v_{\boldsymbol{s}} \in X^{2}
$$

Lemma 2.2. Let Assumption 2.1 hold for Hilbert spaces $X^{1}$ and $X^{2}$. Then for every $v \in \mathcal{L}_{2}\left(X^{2}\right)$,

$$
\lim _{K \rightarrow \infty}\left\|v-\mathcal{S}_{G_{K}} v\right\|_{\mathcal{L}_{2}\left(X^{1}\right)}=0
$$

where $G_{K}:=\left\{(k, s) \in \mathbb{N}_{0} \times \mathbb{F}: 0 \leq k \leq K\right\}$.

Proof. Obviously, by the definition,

$$
\mathcal{S}_{G_{K}} v=\sum_{\boldsymbol{s} \in \mathbb{F}} \sum_{k=0}^{K} \delta_{k}\left(v_{\boldsymbol{s}}\right) H_{\boldsymbol{s}}=\sum_{\boldsymbol{s} \in \mathbb{F}} P_{2^{K}}\left(v_{\boldsymbol{s}}\right) H_{\boldsymbol{s}} .
$$

From Parseval's identity and (2.3) it follows that

$$
\begin{aligned}
\left\|\mathcal{S}_{G_{K}} v\right\|_{\mathcal{L}_{2}\left(X^{1}\right)}^{2} & =\sum_{\boldsymbol{s} \in \mathbb{F}}\left\|P_{2^{K}}\left(v_{\boldsymbol{s}}\right)\right\|_{X^{1}}^{2} \leq 2 \sum_{\boldsymbol{s} \in \mathbb{F}}\left\|v_{\boldsymbol{s}}\right\|_{X^{1}}^{2}+2 \sum_{\boldsymbol{s} \in \mathbb{F}}\left\|v_{\boldsymbol{s}}-P_{2^{K}}\left(v_{\boldsymbol{s}}\right)\right\|_{X^{1}}^{2} \\
& \leq 2 \sum_{\boldsymbol{s} \in \mathbb{F}}\left\|v_{\boldsymbol{s}}\right\|_{X^{1}}^{2}+2 C^{2} 2^{-\alpha K} \sum_{\boldsymbol{s} \in \mathbb{F}}\left\|v_{\boldsymbol{s}}\right\|_{X^{2}}^{2}<\infty .
\end{aligned}
$$

This means that $\mathcal{S}_{G_{K}} v \in \mathcal{L}_{2}\left(X^{1}\right)$. Hence, by Parseval's identity and (2.3) we deduce that

$$
\left\|v-\mathcal{S}_{G_{K}} v\right\|_{\mathcal{L}_{2}\left(X^{1}\right)}^{2}=\sum_{\boldsymbol{s} \in \mathbb{F}}\left\|v_{\boldsymbol{s}}-P_{2^{K}}\left(v_{\boldsymbol{s}}\right)\right\|_{X^{1}}^{2} \leq C^{2} 2^{-2 \alpha K} \sum_{\boldsymbol{s} \in \mathbb{F}}\left\|v_{\boldsymbol{s}}\right\|_{X^{2}}^{2}=C^{2} 2^{-2 \alpha K}\|v\|_{\mathcal{L}_{2}\left(X^{2}\right)}^{2}
$$

which proves the lemma.

Theorem 2.3. Let $0<p \leq 2$. Let Assumption 2.1 hold for Hilbert spaces $X^{1}$ and $X^{2}$. Let $v \in \mathcal{L}_{2}\left(X^{2}\right)$ be represented by the series (2.5). Assume that for $r=1,2$ there exist sequences $\left(\sigma_{r ; \boldsymbol{s}}\right)_{\boldsymbol{s} \in \mathbb{F}}$ of numbers strictly larger than 1 such that

$$
\sum_{\boldsymbol{s} \in \mathbb{F}}\left(\sigma_{r ; \boldsymbol{s}}\left\|v_{\boldsymbol{s}}\right\|_{X^{r}}\right)^{2}<\infty
$$


and $\left(\sigma_{r ; s}^{-1}\right)_{s \in \mathbb{F}} \in \ell_{q_{r}}(\mathbb{F})$ for some $0<q_{1} \leq q_{2}<\infty$. Define for $\xi>0$

$$
G(\xi):=\left\{\begin{array}{lll}
\left\{(k, s) \in \mathbb{N}_{0} \times \mathbb{F}: 2^{k} \sigma_{2 ; s}^{q_{2}} \leq \xi\right\} & \text { if } \quad \alpha \leq 1 / q_{2} \\
\left\{(k, s) \in \mathbb{N}_{0} \times \mathbb{F}: \sigma_{1 ; s}^{q_{1}} \leq \xi, 2^{\alpha q_{1} k} \sigma_{2 ; s}^{q_{1}} \leq \xi\right\} & \text { if } \quad \alpha>1 / q_{2} .
\end{array}\right.
$$

Then for each $n \in \mathbb{N}$ there exists a number $\xi_{n}$ such that $\operatorname{dim}\left(\mathcal{V}\left(G\left(\xi_{n}\right)\right) \leq n\right.$ and

$$
\left\|v-\mathcal{S}_{G\left(\xi_{n}\right)} v\right\|_{\mathcal{L}_{p}\left(X^{1}\right)} \leq C n^{-\min (\alpha, \beta)} .
$$

The rate $\alpha$ corresponds to the approximation of a single function in $X^{2}$ as given by (2.3), and the rate $\beta$ is given by

$$
\beta:=\frac{1}{q_{1}} \frac{\alpha}{\alpha+\delta}, \quad \delta:=\frac{1}{q_{1}}-\frac{1}{q_{2}} .
$$

The constant $C$ in (2.8) is independent of $v$ and $n$.

Proof. Due to the inequality $\|\cdot\|_{\mathcal{L}_{p}\left(X^{1}\right)} \leq\|\cdot\|_{\mathcal{L}_{2}\left(X^{1}\right)}$, it is sufficient to prove the theorem for $p=2$.

We first consider the case $\alpha \leq 1 / q_{2}$. Let $\xi>0$ be given and take arbitrary positive number $\varepsilon$. Since $G(\xi)$ is finite, from the definition of $G_{K}$ and Lemma 2.2 it follows that there exists $K=K(\xi, \varepsilon)$ such that $G(\xi) \subset G_{K}$ and

$$
\left\|v-\mathcal{S}_{G_{K}} v\right\|_{\mathcal{L}_{2}\left(X^{1}\right)} \leq \varepsilon
$$

By the triangle inequality,

$$
\left\|v-\mathcal{S}_{G(\xi)} v\right\|_{\mathcal{L}_{2}\left(X^{1}\right)} \leq\left\|v-\mathcal{S}_{G_{K}} v\right\|_{\mathcal{L}_{2}\left(X^{1}\right)}+\left\|\mathcal{S}_{G_{K}} v-\mathcal{S}_{G(\xi)} v\right\|_{\mathcal{L}_{2}\left(X^{1}\right)}
$$

We have by Parseval's identity and (2.4) that

$$
\begin{aligned}
& \left\|\mathcal{S}_{G_{K}} v-\mathcal{S}_{G(\xi)} v\right\|_{\mathcal{L}_{2}\left(X^{1}\right)}^{2}=\left\|\sum_{\boldsymbol{s} \in \mathbb{F}} \sum_{k=0}^{K} \delta_{k}\left(v_{\boldsymbol{s}}\right) H_{\boldsymbol{s}}-\sum_{\boldsymbol{s} \in \mathbb{F}} \sum_{2^{k} \sigma_{2, s}^{q_{2}} \leq \xi} \delta_{k}\left(v_{\boldsymbol{s}}\right) H_{\boldsymbol{s}}\right\|_{\mathcal{L}_{2}\left(X^{1}\right)}^{2} \\
& =\left\|\sum_{\boldsymbol{s} \in \mathbb{F}} \sum_{\xi \sigma_{2 ; s}^{-q_{2}<2^{k} \leq 2^{K}}} \delta_{k}\left(v_{\boldsymbol{s}}\right) H_{\boldsymbol{s}}\right\|_{\mathcal{L}_{2}\left(X^{1}\right)}^{2}=\sum_{\boldsymbol{s} \in \mathbb{F}}\left\|\sum_{\xi \sigma_{2 ; \boldsymbol{s}}^{-q_{2}<2^{k} \leq 2^{K}}} \delta_{k}\left(v_{\boldsymbol{s}}\right)\right\|_{X^{1}}^{2} \\
& \leq \sum_{\boldsymbol{s} \in \mathbb{F}}\left(\sum_{\xi \sigma_{2 ; s}^{-q_{2}<2^{k} \leq 2^{K}}}\left\|\delta_{k}\left(v_{\boldsymbol{s}}\right)\right\|_{X^{1}}\right)^{2} \leq \sum_{\boldsymbol{s} \in \mathbb{F}}\left(\sum_{\xi \sigma_{2 ; s}^{-q_{2}<2^{k} \leq 2^{K}}} C 2^{-\alpha k}\left\|v_{\boldsymbol{s}}\right\|_{X^{2}}\right)^{2} \\
& \leq C \sum_{\boldsymbol{s} \in \mathbb{F}}\left\|v_{\boldsymbol{s}}\right\|_{X^{2}}^{2}\left(\sum_{2^{k}>\xi \sigma_{2 ; \boldsymbol{s}}^{-q_{2}}} 2^{-\alpha k}\right)^{2} \leq C \sum_{\boldsymbol{s} \in \mathbb{F}}\left\|v_{\boldsymbol{s}}\right\|_{X^{2}}^{2}\left(\xi \sigma_{2 ; \boldsymbol{s}}^{-q_{2}}\right)^{-2 \alpha} .
\end{aligned}
$$

Hence, by the inequalities $q_{2} \alpha \leq 1$ and $\sigma_{2 ; s}>1$ we derive that

$$
\left\|\mathcal{S}_{G_{K}} v-\mathcal{S}_{G(\xi)} v\right\|_{\mathcal{L}_{2}\left(X^{1}\right)}^{2} \leq C \xi^{-2 \alpha} \sum_{\boldsymbol{s} \in \mathbb{F}}\left(\sigma_{2 ; \boldsymbol{s}}\left\|v_{\boldsymbol{s}}\right\|_{X^{2}}\right)^{2}=C \xi^{-2 \alpha} .
$$

Since $\varepsilon>0$ is arbitrary, from the last estimates and (2.10) and (2.11) we derive that

$$
\left\|v-\mathcal{S}_{G(\xi)} v\right\|_{\mathcal{L}_{2}\left(X^{1}\right)} \leq C \xi^{-\alpha}
$$


For the dimension of the space $\mathcal{V}(G(\xi))$ we have that

$$
\begin{aligned}
\operatorname{dim} \mathcal{V}(G(\xi)) & \leq \sum_{(k, \boldsymbol{s}) \in G(\xi)} \operatorname{dim} V_{2^{k}} \leq \sum_{(k, \boldsymbol{s}) \in G(\xi)} 2^{k} \\
& \leq \sum_{\boldsymbol{s} \in \mathbb{F}} \sum_{2^{k} \leq \xi \sigma_{2 ; \boldsymbol{s}}^{-q_{2}}} 2^{k} \leq 2 \sum_{\boldsymbol{s} \in \mathbb{F}} \xi \sigma_{2 ; \boldsymbol{s}}^{-q_{2}}=M \xi,
\end{aligned}
$$

where $M:=2\left\|\left(\sigma_{2 ; s}^{-1}\right)\right\|_{\ell_{q_{2}}(\mathbb{F})}^{q_{2}}$. For any $n \in \mathbb{N}$, letting $\xi_{n}$ be a number satisfying the inequalities

$$
M \xi_{n} \leq n<2 M \xi_{n},
$$

we derive that $\operatorname{dim} \mathcal{V}\left(G\left(\xi_{n}\right)\right) \leq n$. On the other hand, from (2.14) it follows that $\xi_{n}^{-\alpha} \leq(2 M)^{\alpha} n^{-\alpha}$. This together with (2.12) proves that

$$
\left\|v-\mathcal{S}_{G\left(\xi_{n}\right)} v\right\|_{\mathcal{L}_{2}\left(X^{1}\right)} \leq C n^{-\alpha}, \quad \alpha \leq 1 / q_{2} .
$$

We now consider the case $\alpha>1 / q_{2}$. Putting

$$
v_{\xi}:=\sum_{\sigma_{1 ; s}^{q_{1}} \leq \xi} v_{\boldsymbol{s}} H_{\boldsymbol{s}},
$$

we get

$$
\left\|v-\mathcal{S}_{G(\xi)} v\right\|_{\mathcal{L}_{2}\left(X^{1}\right)} \leq\left\|v-v_{\xi}\right\|_{\mathcal{L}_{2}\left(X^{1}\right)}+\left\|v_{\xi}-\mathcal{S}_{G(\xi)} v\right\|_{\mathcal{L}_{2}\left(X^{1}\right)} .
$$

The norms in the right hand side can be estimated using Parseval's identity and the hypothesis of the theorem. Thus, for the norm $\left\|v-v_{\xi}\right\|_{\mathcal{L}_{2}\left(X^{1}\right)}$ we have that

$$
\begin{aligned}
\left\|v-v_{\xi}\right\|_{\mathcal{L}_{2}\left(X^{1}\right)}^{2} & =\sum_{\sigma_{1 ; s}>\xi^{1 / q_{1}}}\left\|v_{\boldsymbol{s}}\right\|_{X^{1}}^{2}=\sum_{\sigma_{1 ; \boldsymbol{s}}>\xi^{1 / q_{1}}}\left(\sigma_{1 ; \boldsymbol{s}}\left\|v_{\boldsymbol{s}}\right\|_{X^{1}}\right)^{2} \sigma_{1 ; \boldsymbol{s}}^{-2} \\
& \leq \xi^{-2 / q_{1}} \sum_{\sigma_{1 ; \boldsymbol{s}}>\xi^{1 / q_{1}}}\left(\sigma_{1 ; \boldsymbol{s}}\left\|v_{\boldsymbol{s}}\right\|_{X^{1}}\right)^{2} \leq C \xi^{-2 / q_{1}} .
\end{aligned}
$$

For the norm $\left\|v_{\xi}-\mathcal{S}_{G(\xi)} v\right\|_{\mathcal{L}_{2}\left(X^{1}\right)}$, with $N=N(\xi, s):=2^{\left\lfloor\log _{2}\left(\xi^{1 / q_{1} \alpha} \sigma_{2 ; s}^{-1 / \alpha}\right)\right\rfloor}$ we obtain

$$
\begin{aligned}
\left\|v_{\xi}-\mathcal{S}_{G(\xi)} v\right\|_{\mathcal{L}_{2}\left(X^{1}\right)}^{2} & =\sum_{\sigma_{1 ; \boldsymbol{s}}>\xi^{1 / q_{1}}}\left\|v_{\boldsymbol{s}}-\sum_{2^{\alpha q_{1} k} \sigma_{2 ; \boldsymbol{s}}^{q_{1}} \leq \xi} \delta_{k}\left(v_{\boldsymbol{s}}\right)\right\|_{X^{1}}^{2} \leq \sum_{\boldsymbol{s} \in \mathbb{F}}\left\|v_{\boldsymbol{s}}-P_{N}\left(v_{\boldsymbol{s}}\right)\right\|_{X^{1}}^{2} \\
& \leq \sum_{\boldsymbol{s} \in \mathbb{F}} C N^{-2 \alpha}\left\|v_{\boldsymbol{s}}\right\|_{X^{2}}^{2} \leq C \xi^{-2 / q_{1}} \sum_{\boldsymbol{s} \in \mathbb{F}}\left(\sigma_{2 ; \boldsymbol{s}}\left\|v_{\boldsymbol{s}}\right\|_{X^{2}}\right)^{2} \leq C \xi^{-2 / q_{1}}
\end{aligned}
$$

These estimates yield that

$$
\left\|v-\mathcal{S}_{G(\xi)} v\right\|_{\mathcal{L}_{2}\left(X^{1}\right)} \leq C \xi^{-1 / q_{1}}
$$


For the dimension of the space $\mathcal{V}(G(\xi))$, with $q:=q_{2} \alpha>1$ and $1 / q^{\prime}+1 / q=1$ we have that

$$
\begin{aligned}
\operatorname{dim} \mathcal{V}(G(\xi)) & \leq \sum_{(k, s) \in G(\xi)} \operatorname{dim} V_{2^{k}} \leq \sum_{\sigma_{1 ; s}^{q_{1} \leq \xi}} \sum_{2^{\alpha q_{1} k} \sigma_{2 ; s}^{q_{1}} \leq \xi} 2^{k} \\
& \leq 2 \sum_{\sigma_{1 ; s}^{q_{1} \leq \xi}} \xi^{1 /\left(q_{1} \alpha\right)} \sigma_{2 ; \boldsymbol{s}}^{-1 / \alpha} \leq 2 \xi^{1 /\left(q_{1} \alpha\right)}\left(\sum_{\sigma_{1 ; s}^{q_{1} \leq \xi}} \sigma_{2 ; \boldsymbol{s}}^{-q_{2}}\right)^{1 / q}\left(\sum_{\sigma_{1 ; s}^{q_{1} \leq \xi}} 1\right)^{1 / q^{\prime}} \\
& \leq 2 \xi^{1 /\left(q_{1} \alpha\right)}\left(\sum_{\boldsymbol{s} \in \mathbb{F}} \sigma_{2 ; s}^{-q_{2}}\right)^{1 / q}\left(\sum_{\boldsymbol{s} \in \mathbb{F}} \xi \sigma_{1 ; s}^{-q_{1}}\right)^{1 / q^{\prime}}=M \xi^{1+\delta / \alpha}
\end{aligned}
$$

where $M:=2\left\|\left(\sigma_{2 ; s}^{-1}\right)\right\|_{\ell_{q_{2}}(\mathbb{F})}^{q_{2} / q}\left\|\left(\sigma_{1 ; s}^{-1}\right)\right\|_{\ell_{q_{1}}(\mathbb{F})}^{q_{1} / q^{\prime}}$. For any $n \in \mathbb{N}$, letting $\xi_{n}$ be a number satisfying the inequalities

$$
M \xi_{n}^{1+\delta / \alpha} \leq n<2 M \xi_{n}^{1+\delta / \alpha},
$$

we derive that $\operatorname{dim} \mathcal{V}\left(G\left(\xi_{n}\right)\right) \leq n$. On the other hand, by (2.18),

$$
\xi_{n}^{-1 / q_{1}} \leq(2 M)^{\frac{\alpha}{\alpha+\delta}} n^{-\frac{1}{q_{1}} \frac{\alpha}{\alpha+\delta}} .
$$

This together with (2.17) proves that

$$
\left\|v-\mathcal{S}_{G\left(\xi_{n}\right)} v\right\|_{\mathcal{L}_{2}\left(X^{1}\right)} \leq C n^{-\beta}, \quad \alpha>1 / q_{2} .
$$

By combining the last estimate and (2.15) we obtain (2.8).

Remark 2.4. Let us compare the non-adaptive fully discrete method constructed in Theorem 2.3 with adaptive one considered in Theorem 3.1 of [1]. Both the methods give the same convergence rate $\min (\alpha, \beta)$. However, the ways to form them are different. Let us explain this.

In Theorem 3.1 of [1] for the lognormal case, for a given $v \in X^{2}$, a preliminary polynomial approximation $v_{m}:=\sum_{s \in \Lambda_{m}} v_{s} H_{s}$ is taken by truncation of the Hermite gpc expansion (2.5), where $\Lambda_{m} \subset \mathbb{F}$ is a set corresponding to $m$ largest $\left\|v_{\boldsymbol{s}}\right\|_{X^{1}}$. The coefficients $v_{\boldsymbol{s}} \in X^{2}$ then is approximated by $v_{\boldsymbol{s}, m_{\boldsymbol{s}}}:=P_{m_{\boldsymbol{s}}}\left(v_{\boldsymbol{s}}\right)$, and $v$ is approximated by the resulting approximant $v_{\boldsymbol{m}}:=\sum_{\boldsymbol{s} \in \Lambda_{m}} v_{\boldsymbol{s}, m_{\boldsymbol{s}}} H_{\boldsymbol{s}}$. An optimal choice of $\left(m_{\boldsymbol{s}}\right)_{\boldsymbol{s} \in \Lambda_{m}}$ give the rate $\min (\alpha, \beta)$ in terms of $n$ where $n=\sum_{s \in \Lambda_{m}} m_{s}$ is the dimension of the space $\mathcal{V}_{\boldsymbol{m}} \subset X^{2}$ of all functions of the form $\sum_{\boldsymbol{s} \in \Lambda_{m}} v_{\boldsymbol{s}} H_{\boldsymbol{s}}, v_{\boldsymbol{s}} \in V_{m_{\boldsymbol{s}}}$. This is an adaptive approximation method, since the choice of $m$ largest $\left\|v_{\boldsymbol{s}}\right\|_{X^{1}}$ essentially depends of $v$.

In Theorem 2.3, the approximant $\mathcal{S}_{G\left(\xi_{n}\right)} v$ belongs to the space $\mathcal{V}\left(G\left(\xi_{n}\right)\right) \subset \mathcal{V}\left(X^{2}\right)$. The convergence rate $\min (\alpha, \beta)$ of approximation by $\mathcal{S}_{G\left(\xi_{n}\right)} v$ is given in terms of $n$ where the thresholding parameter $\xi_{n}$ is chosen such that $\operatorname{dim}\left(\mathcal{V}\left(G\left(\xi_{n}\right)\right) \leq n\right.$. Notice that $\mathcal{S}_{G\left(\xi_{n}\right)} v=\sum_{\boldsymbol{s} \in \Lambda} v_{\boldsymbol{s}, m_{s}} H_{\boldsymbol{s}}$ and the space $\mathcal{V}\left(G\left(\xi_{n}\right)\right)$ consists of all functions of the form $\sum_{\boldsymbol{s} \in \Lambda} v_{s} H_{s}, v_{s} \in V_{m_{s}}$, for a certain set $\Lambda$ depending on $n$, i.e., formally they are similar to those in Theorem 3.1 of [1]. The difference here is that the set $\Lambda$ is defined independently of $v$. Hence, our approximation methods are non-adaptive provided that there is a sequence $\left(P_{n}\right)_{n \in \mathbb{N}_{0}}$ of linear operators from $X^{1}$ into $n$-dimensional subspaces $V_{n} \subset X^{1}$ satisfying (2.3) for all $v \in X^{2}$ (Assumption 2.1). See also Remark 3.2 of $[1]$.

\section{Polynomial interpolation approximation}

In this section, we construct general linear fully discrete polynomial interpolation methods of approximation in the Bochner space $\mathcal{L}_{p}\left(X^{1}\right)$ of functions taking values in $X^{2}$ and having a weighted $\ell_{2}$-summability of Hermite expansion coefficients for Hilbert spaces $X^{1}$ and $X^{2}$ satisfying a certain "spatial" approximation property. In particular, we prove convergence rates for these methods of approximation. We also briefly consider linear non-fully discrete polynomial interpolation methods of approximation. 


\subsection{Auxiliary results}

Let $w=\exp (-Q)$, where $Q$ is an even function on $\mathbb{R}$ and $y Q^{\prime}(y)$ is positive and increasing in $(0, \infty)$, with limits 0 and $\infty$ at 0 and $\infty$. Notice that for the standard Gaussian density $g$ defined in $(2.1), \sqrt{g}$ is such a function. For $m \in \mathbb{N}$, the $n$th Mhaskar-Rakhmanov-Saff number $a_{m}=a_{m}(w)$ is defined as the positive root of the equation

$$
m=\frac{2}{\pi} \int_{0}^{1} \frac{a_{m} y Q^{\prime}\left(a_{m} y\right)}{\sqrt{1-y^{2}}} \mathrm{~d} y .
$$

From Page 11 of [25] we have for $w=\sqrt{g}$,

$$
a_{m}(\sqrt{g})=\sqrt{m}
$$

For $0<p, q \leq \infty$, we introduce the quantity

$$
\delta(p, q):=\frac{1}{2}\left|\frac{1}{p}-\frac{1}{q}\right| .
$$

Lemma 3.1. Let $0<p, q \leq \infty$. Then there exists a positive constant $C_{p, q}$ such that for every polynomial $\varphi$ of degree $\leq m$, the Nikol'skii-type inequality holds

$$
\|\varphi \sqrt{g}\|_{L_{p}(\mathbb{R})} \leq C_{p, q} m^{\delta(p, q)}\|\varphi \sqrt{g}\|_{L_{q}(\mathbb{R})} .
$$

Proof. This lemma is an immediate consequence of (3.1) and the inequality

$$
\|\varphi \sqrt{g}\|_{L_{p}(\mathbb{R})} \leq C_{p, q} N_{m}(p, q)\|\varphi \sqrt{g}\|_{L_{q}(\mathbb{R})}
$$

which follows from Theorem 9.1 and p. 61 of [25], where

$$
N_{m}(p, q):= \begin{cases}a_{m}^{1 / p-1 / q}, & p<q \\ \left(\frac{n}{a_{m}}\right)^{1 / q-1 / p}, & p>q .\end{cases}
$$

Lemma 3.2. We have

$$
\left\|H_{m} \sqrt{g}\right\|_{L_{\infty}(\mathbb{R})} \leq 1, \quad m \in \mathbb{N}_{0} .
$$

Proof. From Cramér's bound (see, e.g., [17], Page 208, (19)) we have for every $m \in \mathbb{N}_{0}$ and every $x \in \mathbb{R}$, $\left|H_{m}(x) \sqrt{g(x)}\right| \leq K(2 \pi)^{-1 / 4}$, where $K:=1.086435$. This implies (3.2).

For our application the estimate (3.2) is sufficient, see [13] for an improvement.

For $\theta, \lambda \geq 0$, we define the sequence

$$
p_{\boldsymbol{s}}(\theta, \lambda):=\prod_{j \in \mathbb{N}}\left(1+\lambda s_{j}\right)^{\theta}, \quad s \in \mathbb{F} .
$$

Lemma 3.3. Let $0<p \leq 2$ and $X$ be a Hilbert space. Let $v \in L_{2}\left(\mathbb{R}^{\infty}, X, \gamma\right)$ be represented by the series (2.2). Assume that there exists a sequence $\boldsymbol{\sigma}=\left(\sigma_{\boldsymbol{s}}\right)_{\boldsymbol{s} \in \mathbb{F}}$ of positive numbers such that

$$
\sum_{\boldsymbol{s} \in \mathbb{F}}\left(\sigma_{\boldsymbol{s}}\left\|v_{\boldsymbol{s}}\right\|_{X}\right)^{2}<\infty
$$


We have the following.

(i) If $\left(p_{\boldsymbol{s}}(\theta, \lambda) \sigma_{\boldsymbol{s}}^{-1}\right)_{\boldsymbol{s} \in \mathbb{F}} \in \ell_{q}(\mathbb{F})$ for some $0<q \leq 2$ and $\theta, \lambda \geq 0$, then $\left(p_{\boldsymbol{s}}(\theta, \lambda)\left\|v_{\boldsymbol{s}}\right\|_{X}\right)_{\boldsymbol{s} \in \mathbb{F}} \in \ell_{\bar{q}}(\mathbb{F})$ for $\bar{q}$ such that $1 / \bar{q}=1 / 2+1 / q$.

(ii) If $\left(\sigma_{\boldsymbol{s}}^{-1}\right)_{\boldsymbol{s} \in \mathbb{F}} \in \ell_{q}(\mathbb{F})$ for some $0<q \leq 2$, then the series $(2.2)$ converges absulutely in $\mathcal{L}_{p}(X)$ to $v$.

Proof. Since $\tau:=2 / \bar{q} \geq 1$, with $1 / \tau+1 / \tau^{\prime}=1$ and $p_{\boldsymbol{s}}=p_{\boldsymbol{s}}(\theta, \lambda)$ by the Hölder inequality we have that

$$
\begin{aligned}
\sum_{\boldsymbol{s} \in \mathbb{F}}\left(p_{\boldsymbol{s}}\left\|v_{\boldsymbol{s}}\right\|_{X}\right)^{\bar{q}} & \leq\left(\sum_{\boldsymbol{s} \in \mathbb{F}}\left(\sigma_{\boldsymbol{s}}^{\bar{q}}\left\|v_{\boldsymbol{s}}\right\|_{X}^{\bar{q}}\right)^{\tau}\right)^{1 / \tau}\left(\sum_{\boldsymbol{s} \in \mathbb{F}}\left(p_{\boldsymbol{s}}^{\bar{q}} \sigma_{\boldsymbol{s}}^{-\bar{q}}\right)^{\tau^{\prime}}\right)^{1 / \tau^{\prime}} \\
& =\left(\sum_{\boldsymbol{s} \in \mathbb{F}}\left(\sigma_{\boldsymbol{s}}\left\|v_{\boldsymbol{s}}\right\|_{X}\right)^{2}\right)^{\bar{q} / 2}\left(\sum_{\boldsymbol{s} \in \mathbb{F}}\left(p_{\boldsymbol{s}} \sigma_{\boldsymbol{s}}^{-1}\right)^{q}\right)^{1-\bar{q} / 2}<\infty .
\end{aligned}
$$

This proves the assersion (i).

We have by the inequality $\bar{q} \leq 1$ and (i) for $\theta=\lambda=0$,

$$
\begin{aligned}
\sum_{\boldsymbol{s} \in \mathbb{F}}\left\|v_{\boldsymbol{s}} H_{\boldsymbol{s}}\right\|_{\mathcal{L}_{2}(X)} & =\sum_{\boldsymbol{s} \in \mathbb{F}}\left\|v_{\boldsymbol{s}}\right\|_{X}\left\|H_{\boldsymbol{s}}\right\|_{L_{2}\left(\mathbb{R}^{\infty}, \gamma\right)} \\
& \leq \sum_{\boldsymbol{s} \in \mathbb{F}}\left\|v_{\boldsymbol{s}}\right\|_{X} \leq\left(\sum_{\boldsymbol{s} \in \mathbb{F}}\left(\left\|v_{\boldsymbol{s}}\right\|_{X}\right)^{\bar{q}}\right)^{1 / \bar{q}}<\infty .
\end{aligned}
$$

This yields that the series (2.2) absolutely converges in $\mathcal{L}_{2}(X)$ to $v$, since by the assumption this series converges in $\mathcal{L}_{2}(X)$ to $v$. The assertion (ii) is proven for the case $p=2$. The case $0<p<2$ is derived from the case $p=2$ and the inequality $\|\cdot\|_{\mathcal{L}_{p}(X)} \leq\|\cdot\|_{\mathcal{L}_{2}(X)}$.

Lemma 3.4. Let $0<p \leq 2$. Let Assumption 2.1 hold for Hilbert spaces $X^{1}$ and $X^{2}$, and let the assumptions of Lemma 3.3(ii) hold for the space $X^{1}$. Then every $v \in \mathcal{L}_{2}\left(X^{2}\right)$ can be represented as the series

$$
v=\sum_{(k, \boldsymbol{s}) \in \mathbb{N}_{0} \times \mathbb{F}} \delta_{k}\left(v_{\boldsymbol{s}}\right) H_{\boldsymbol{s}}
$$

converging absolutely in $\mathcal{L}_{p}\left(X^{1}\right)$ to $v$.

Proof. This lemma can be proven in a way similar to the proof of Lemma 2.1 in [16]. For completeness, let us give a detailed proof. As in the proof of Lemma 3.3, it is sufficient to prove the lemma for the case $p=2$. Put $v_{k, \boldsymbol{s}}(\boldsymbol{y})(\boldsymbol{x}):=\delta_{k}\left(v_{\boldsymbol{s}}\right)(\boldsymbol{x}) H_{\boldsymbol{s}}(\boldsymbol{y})$. It is well known that the unconditional convergence in a Banach space follows from the absolute convergence. Using this fact, from Lemma 3.3(ii) and Assumption 2.1 we derive that on one hand, the series $\sum_{\boldsymbol{s} \in \mathbb{F}} v_{k, \boldsymbol{s}}(\boldsymbol{y})(\boldsymbol{x})$ converges unconditionally in $\mathcal{L}_{2}\left(X^{1}\right)$, and uniformly for $k \in \mathbb{N}_{0}$, and on the other hand, the series $\sum_{k \in \mathbb{N}_{0}} v_{k, s}(\boldsymbol{y})(\boldsymbol{x})$ converges absolutely in $\mathcal{L}_{2}\left(X^{1}\right)$, and uniformly for $s \in \mathbb{F}$ to $v_{\boldsymbol{s}}(\boldsymbol{x}) H_{\boldsymbol{s}}(\boldsymbol{y})$. Hence, since the series $(2.5)$ converges unconditionally in $\mathcal{L}_{2}\left(X^{1}\right)$, we have that

$$
v(\boldsymbol{y})(\boldsymbol{x})=\sum_{\boldsymbol{s} \in \mathbb{F}} v_{\boldsymbol{s}}(\boldsymbol{x}) H_{\boldsymbol{s}}(\boldsymbol{y})=\sum_{\boldsymbol{s} \in \mathbb{F}} \sum_{k \in \mathbb{N}_{0}} v_{k, \boldsymbol{s}}(\boldsymbol{y})(\boldsymbol{x})=\sum_{(k, \boldsymbol{s}) \in \mathbb{N}_{0} \times \mathbb{F}} v_{k, \boldsymbol{s}}(\boldsymbol{y})(\boldsymbol{x}), \quad \boldsymbol{x} \in D, \boldsymbol{y} \in \mathbb{R}^{\infty},
$$

where the last series converges unconditionally in $\mathcal{L}_{2}\left(X^{1}\right)$. This means that the series in (3.4) converges absolutely to $v$, since by Lemma 2.6 the sum $S_{G_{K}}$ converges in $\mathcal{L}_{2}\left(X^{1}\right)$ to $v$ when $K \rightarrow \infty$. 
We will need the following two lemmata for application in estimating the convergence rate of the fully discrete polynomial interpolation approximation in this section and of integration in Section 4.

Lemma 3.5. Under the hypothesis of Theorem 2.3, assume in addition that $q_{1}<2$. Define for $\xi>0$

$$
G(\xi):= \begin{cases}\left\{(k, s) \in \mathbb{N}_{0} \times \mathbb{F}: 2^{k} \sigma_{2, \boldsymbol{s}}^{q_{2}} \leq \xi\right\} & \text { if } \quad \alpha \leq 1 / q_{2}-1 / 2 \\ \left\{(k, s) \in \mathbb{N}_{0} \times \mathbb{F}: \sigma_{1 ; \boldsymbol{s}}^{q_{1}} \leq \xi, 2^{(\alpha+1 / 2) k} \sigma_{2 ; \boldsymbol{s}} \leq \xi^{\vartheta}\right\} & \text { if } \quad \alpha>1 / q_{2}-1 / 2\end{cases}
$$

where

$$
\vartheta:=1 / q_{1}+\left(1 / q_{1}-1 / q_{2}\right) /(2 \alpha)
$$

Then for each $\xi>0$,

$$
\left\|v-\mathcal{S}_{G(\xi)} v\right\|_{\mathcal{L}_{p}\left(X^{1}\right)} \leq C \times\left\{\begin{array}{lll}
\xi^{-\alpha} & \text { if } & \alpha \leq 1 / q_{2}-1 / 2 \\
\xi^{-\left(1 / q_{1}-1 / 2\right)} & \text { if } & \alpha>1 / q_{2}-1 / 2
\end{array}\right.
$$

The rate $\alpha$ is given by (2.3). The constant $C$ in (3.7) is independent of $v$ and $\xi$.

Proof. Similarly to the proof of Lemma 3.3, it is sufficient to prove the lemma for $p=2$. Since in the case $\alpha \leq 1 / q_{2}-1 / 2$, the formulas (2.7) and (3.5) define the same set $G(\xi)$ for $\xi>0$, from (2.12) follows the lemma for this case. Let us consider the case $\alpha>1 / q_{2}-1 / 2$. Putting

$$
v_{\xi}:=\sum_{\sigma_{1 ; s}^{q_{1}} \leq \xi} v_{s} H_{\boldsymbol{s}}
$$

we get

$$
\left\|v-\mathcal{S}_{G(\xi)} v\right\|_{\mathcal{L}_{2}\left(X^{1}\right)} \leq\left\|v-v_{\xi}\right\|_{\mathcal{L}_{2}\left(X^{1}\right)}+\left\|v_{\xi}-\mathcal{S}_{G(\xi)} v\right\|_{\mathcal{L}_{2}\left(X^{1}\right)}
$$

As in the proof of Lemma 3.4, by Lemma 3.3(ii) the series $(2.2)$ converges unconditionally in $\mathcal{L}_{2}\left(X^{1}\right)$ to $v$. Hence the norm $\left\|v-v_{\xi}\right\|_{\mathcal{L}_{2}\left(X^{1}\right)}$ can be estimated by

$$
\begin{aligned}
\left\|v-v_{\xi}\right\|_{\mathcal{L}_{2}\left(X^{1}\right)} & \leq \sum_{\sigma_{1 ; \boldsymbol{s}}>\xi^{1 / q_{1}}}\left\|v_{\boldsymbol{s}}\right\|_{X^{1}}\left\|H_{\boldsymbol{s}}\right\|_{L_{2}\left(\mathbb{R}^{\infty}, \gamma\right)}=\sum_{\sigma_{1 ; \boldsymbol{s}}>\xi^{1 / q_{1}}}\left\|v_{\boldsymbol{s}}\right\|_{X^{1}} \\
& \leq\left(\sum_{\sigma_{1 ; \boldsymbol{s}}>\xi^{1 / q_{1}}}\left(\sigma_{1 ; \boldsymbol{s}}\left\|v_{\boldsymbol{s}}\right\|_{\left.X^{1}\right)^{2}}\right)^{1 / 2}\left(\sum_{\sigma_{1 ; \boldsymbol{s}}>\xi^{1 / q_{1}}} \sigma_{1 ; \boldsymbol{s}}^{-2}\right)^{1 / 2}\right. \\
& \leq C\left(\sum_{\sigma_{1 ; \boldsymbol{s}}>\xi^{1 / q_{1}}} \sigma_{1 ; \boldsymbol{s}}^{-q_{1}} \sigma_{1 ; \boldsymbol{s}}^{-\left(2-q_{1}\right)}\right)^{1 / 2} \\
& \leq C \xi^{-\left(1 / q_{1}-1 / 2\right)}\left(\sum_{\boldsymbol{s} \in \mathbb{F}} \sigma_{1 ; \boldsymbol{s}}^{-q_{1}}\right)^{1 / 2} \leq C \xi^{-\left(1 / q_{1}-1 / 2\right)}
\end{aligned}
$$


For the norm $\left\|v_{\xi}-\mathcal{S}_{G(\xi)} v\right\|_{\mathcal{L}_{2}\left(X^{1}\right)}$, with $N=N(\xi, s):=2^{\left\lfloor\log _{2}\left(\sigma_{2 ; s}^{-(\alpha+1 / 2)^{-1}} \xi^{\vartheta(\alpha+1 / 2)^{-1}}\right)\right\rfloor}$ we have that

$$
\begin{aligned}
& \left\|v_{\xi}-\mathcal{S}_{G(\xi)} v\right\|_{\mathcal{L}_{2}\left(X^{1}\right)} \leq \sum_{\sigma_{1 ; s}^{q_{1}} \leq \xi}\left\|v_{\boldsymbol{s}}-\sum_{2^{\alpha q_{1} k} \sigma_{2 ; \boldsymbol{s}}^{q_{1}} \leq \xi} \delta_{k}\left(v_{\boldsymbol{s}}\right)\right\|_{X^{1}}\left\|H_{\boldsymbol{s}}\right\|_{L_{2}\left(\mathbb{R}^{\infty}, \gamma\right)} \\
& =C \sum_{\sigma_{1 ; s}^{q_{1}} \leq \xi}\left\|v_{\boldsymbol{s}}-P_{N}\left(v_{\boldsymbol{s}}\right)\right\|_{X^{1}} \leq C \sum_{\sigma_{1 ; s}^{q_{1}} \leq \xi} N^{-\alpha}\left\|v_{\boldsymbol{s}}\right\|_{X^{2}} \\
& \leq C \sum_{\sigma_{1 ; \boldsymbol{s}}^{q_{1} \leq \xi}}\left(\xi^{1 /\left(q_{1} \alpha\right)} \sigma_{2 ; \boldsymbol{s}}^{-1 / \alpha}\right)^{-\alpha}\left\|v_{\boldsymbol{s}}\right\|_{X^{2}} \leq C \xi^{-1 / q_{1}} \sum_{\sigma_{1 ; \boldsymbol{s}}^{q_{1}} \leq \xi} \sigma_{2 ; \boldsymbol{s}}\left\|v_{\boldsymbol{s}}\right\|_{X^{2}} \\
& \leq C \xi^{-1 / q_{1}}\left(\sum_{\sigma_{1 ; s}^{q_{1}} \leq \xi}\left(\sigma_{2 ; s}\left\|v_{s}\right\|_{X^{2}}\right)^{2}\right)^{1 / 2}\left(\sum_{\sigma_{1 ; s}^{q_{1}} \leq \xi} 1\right)^{1 / 2} \\
& \leq C \xi^{-1 / q_{1}}\left(\sum_{\sigma_{1 ; s}^{q_{1} \leq \xi}} \sigma_{1 ; s}^{-q_{1}} \xi\right)^{1 / 2} \leq C \xi^{-\left(1 / q_{1}-1 / 2\right)}\left(\sum_{s \in \mathbb{F}} \sigma_{1 ; s}^{-q_{1}}\right)^{1 / 2} \leq C \xi^{-\left(1 / q_{1}-1 / 2\right)} .
\end{aligned}
$$

This, (3.8) and (3.9) prove the lemma for the case $\alpha>1 / q_{2}-1 / 2$.

We make use the notation: $\mathbb{F}_{\text {ev }}:=\left\{s \in \mathbb{F}: s_{j}\right.$ even, $\left.j \in \mathbb{N}\right\}$. The following lemma can be proven in a similar way.

Lemma 3.6. Let $0<p \leq 2$. Let Assumption 2.1 hold for Hilbert spaces $X^{1}$ and $X^{2}$. Let $v \in \mathcal{L}_{2}\left(X^{2}\right)$ be represented by the series

$$
v=\sum_{s \in \mathbb{F}_{\mathrm{ev}}} v_{\boldsymbol{s}} H_{\boldsymbol{s}}, \quad v_{\boldsymbol{s}} \in X^{2}
$$

Assume that for $r=1,2$ there exist sequences $\left(\sigma_{r ; s}\right)_{s \in \mathbb{F}_{\mathrm{ev}}}$ of numbers strictly larger than 1 such that

$$
\sum_{\boldsymbol{s} \in \mathbb{F}_{\mathrm{ev}}}\left(\sigma_{r ; \boldsymbol{s}}\left\|v_{\boldsymbol{s}}\right\|_{X^{r}}\right)^{2}<\infty
$$

and $\left(\sigma_{r ; \boldsymbol{s}}^{-1}\right)_{\boldsymbol{s} \in \mathbb{F}_{\mathrm{ev}}} \in \ell_{q_{r}}\left(\mathbb{F}_{\mathrm{ev}}\right)$ for some $0<q_{1} \leq q_{2}<\infty$ with $q_{1}<2$. Define for $\xi>0$,

$$
G_{\mathrm{ev}}(\xi):=G(\xi) \cap\left(\mathbb{N}_{0} \times \mathbb{F}_{\mathrm{ev}}\right)= \begin{cases}\left\{(k, s) \in \mathbb{N}_{0} \times \mathbb{F}_{\mathrm{ev}}: 2^{k} \sigma_{2 ; s}^{q_{2}} \leq \xi\right\} & \text { if } \alpha \leq 1 / q_{2}-1 / 2 \\ \left\{(k, s) \in \mathbb{N}_{0} \times \mathbb{F}_{\mathrm{ev}}: \sigma_{1 ; \boldsymbol{s}}^{q_{1}} \leq \xi, 2^{(\alpha+1 / 2) k} \sigma_{2 ; \boldsymbol{s}} \leq \xi^{\vartheta}\right\} & \text { if } \alpha>1 / q_{2}-1 / 2\end{cases}
$$

where $\vartheta$ is as in (3.6). Then for each $\xi>0$,

$$
\left\|v-\mathcal{S}_{G_{\mathrm{ev}}\left(\xi_{n}\right)} v\right\|_{\mathcal{L}_{p}\left(X^{1}\right)} \leq C \times \begin{cases}\xi^{-\alpha} & \text { if } \quad \alpha \leq 1 / q_{2}-1 / 2 \\ \xi^{-\left(1 / q_{1}-1 / 2\right)} & \text { if } \quad \alpha>1 / q_{2}-1 / 2\end{cases}
$$

The rate $\alpha$ is given by (2.3). The constant $C$ in (3.12) is independent of $v$ and $\xi$. 


\subsection{Interpolation approximation}

For every $m \in \mathbb{N}_{0}$, let $Y_{m}=\left(y_{m ; k}\right)_{k=0}^{m}$ be a sequence of points in $\mathbb{R}$ such that

$$
-\infty<y_{m ; 0}<\cdots<y_{m ; m-1}<y_{m ; m}<+\infty ; \quad y_{0 ; 0}=0 .
$$

If $v$ is a function on $\mathbb{R}$ taking value in a Hilbert space $X$ and $m \in \mathbb{N}_{0}$, we define the function $I_{m}(v)$ on $\mathbb{R}$ taking value in $X$ by

$$
I_{m}(v)(y):=\sum_{k=0}^{m} v\left(y_{m ; k}\right) \ell_{m ; k}, \quad \ell_{m ; k}(y):=\prod_{j=0, j \neq k}^{n} \frac{y-y_{m ; j}}{y_{m ; k}-y_{m ; j}}, \quad y \in \mathbb{R},
$$

interpolating $v$ at $y_{m ; k}$, i.e., $I_{m}(v)\left(y_{m ; k}\right)=v\left(y_{m ; k}\right)$. Notice that for a function $v: \mathbb{R} \rightarrow \mathbb{R}$, the function $I_{m}(v)$ is the Lagrange polynomial having degree $\leq m$, and that $I_{m}(\varphi)=\varphi$ for every polynomial $\varphi$ of degree $\leq m$.

Let

$$
\lambda_{m}\left(Y_{m}\right):=\sup _{\|v \sqrt{g}\|_{L_{\infty}(\mathbb{R})} \leq 1}\left\|I_{m}(v) \sqrt{g}\right\|_{L_{\infty}(\mathbb{R})}
$$

be the Lebesgue constant, see, e.g., Page 78 of [25]. We want to choose a sequence $\left(Y_{m}\right)_{m=0}^{\infty}$ so that for some positive numbers $\tau$ and $C$, there holds the inequlity

$$
\lambda_{m}\left(Y_{m}\right) \leq(C m+1)^{\tau}, \quad \forall m \in \mathbb{N}_{0} .
$$

We present two examples of $\left(Y_{m}\right)_{m=0}^{\infty}$ satisfying (3.15). The first example is $\left(Y_{m}^{*}\right)_{n=0}^{\infty}$ where $Y_{m}^{*}=\left(y_{m ; k}^{*}\right)_{k=0}^{n}$ are the strictly increasing sequences of the roots of $H_{m+1}$. Indeed, it was proven by Matjila and Szabados $[26,27,30]$ that

$$
\lambda_{m}\left(Y_{m}^{*}\right) \leq C(m+1)^{1 / 6}, \quad m \in \mathbb{N},
$$

for some positive constant $C$ independent of $n$ (with the obvious inequality $\lambda_{0}\left(Y_{0}^{*}\right) \leq 1$ ). Hence, for every $\varepsilon>0$, there exists a positive constant $C_{\varepsilon}$ independent of $n$ such that

$$
\lambda_{m}\left(Y_{m}^{*}\right) \leq\left(C_{\varepsilon} m+1\right)^{1 / 6+\varepsilon}, \quad \forall m \in \mathbb{N}_{0} .
$$

The minimum distance between consecutive roots $d_{m+1}$ satisfies the inequalities $\frac{\pi \sqrt{2}}{\sqrt{2 m+3}}<d_{m+1}<\frac{\sqrt{21}}{\sqrt{2 m+3}}$, see pp. 130-131 of [31]. The sequences $Y_{m}^{*}$ have been used in [4] for sparse quadrature for non-fully discrete integration with the measure $\gamma$, and in [18] non-fully discrete polynomial interpolation approximation with the measure $\gamma$.

The inequality (3.16) can be improved if $Y_{m-2}^{*}$ is slightly modified by the "method of adding points" suggested by Szabados [30] (for details, see also [25], Sect. 11). More precisely, for $n>2$, he added to $Y_{m-2}^{*}$ two points $\pm \zeta_{m-1}$, near $\pm a_{m-1}(g)$, which are defined by the condition $\left|H_{m-1} \sqrt{g}\right|\left(\zeta_{m-1}\right)=\left\|H_{m-1} \sqrt{g}\right\|_{L_{\infty}(\mathbb{R})}$. By this way, he obtained the strictly increasing sequences

$$
\bar{Y}_{m}^{*}:=\left\{-\zeta_{m}, y_{m-2 ; 0}^{*}, \ldots, y_{m-2, m-2}^{*}, \zeta_{m}\right\}
$$

for which the sequence $\left(\bar{Y}_{m}^{*}\right)_{n=0}^{\infty}$ satisfies the inequality

$$
\lambda_{m}\left(\bar{Y}_{m}^{*}\right) \leq C \log (m-1) \quad(m>2)
$$

which yields that for every $\varepsilon>0$, there exists a positive constant $C_{\varepsilon}$ independent of $n$ such that

$$
\lambda_{m}\left(\bar{Y}_{m}^{*}\right) \leq\left(C_{\varepsilon} m+1\right)^{\varepsilon}, \quad \forall m \in \mathbb{N}_{0} .
$$

For a given sequence $\left(Y_{m}\right)_{m=0}^{\infty}$, we define the univariate operator $\Delta_{m}^{I}$ for $m \in \mathbb{N}_{0}$ by

$$
\Delta_{m}^{I}:=I_{m}-I_{m-1}
$$

with the convention $I_{-1}=0$. 
Lemma 3.7. Assume that $\left(Y_{m}\right)_{m=0}^{\infty}$ is a sequence satisfying the condition (3.15) for some positive numbers $\tau$ and $C$. Then for every $\varepsilon>0$, there exists a positive constant $C_{\varepsilon}$ independent of $m$ such that for every function $v$ on $\mathbb{R}$,

$$
\left\|\Delta_{m}^{I}(v) \sqrt{g}\right\|_{L_{\infty}(\mathbb{R})} \leq\left(C_{\varepsilon} m+1\right)^{\tau+\varepsilon}\|v \sqrt{g}\|_{L_{\infty}(\mathbb{R})}, \quad \forall m \in \mathbb{N}_{0},
$$

whenever the norm in the right-hand side is finite.

Proof. From the assumptions we have that

$$
\left\|\Delta_{m}^{I}(v) \sqrt{g}\right\|_{L_{\infty}(\mathbb{R})} \leq 2(C m+1)^{\tau}\|v \sqrt{g}\|_{L_{\infty}(\mathbb{R})}, \quad \forall m \in \mathbb{N}_{0}
$$

which similarly to (3.16) gives (3.17).

We are interested in sparse-grid interpolation approximation and integration of functions $v$ from the space $\mathcal{L}_{2}(X)$. In order to have a correct definition of interpolation operator let us impose some necessary restrictions on $v$. Let $\mathcal{E}$ be a $\gamma$-measurable subset in $\mathbb{R}^{\infty}$ such that $\gamma(\mathcal{E})=1$ and $\mathcal{E}$ contains all $\boldsymbol{y} \in \mathbb{R}^{\infty}$ with $|\boldsymbol{y}|_{0}<\infty$, where $|\boldsymbol{y}|_{0}$ denotes the number of nonzero components $y_{j}$ of $\boldsymbol{y}$. For a given $\mathcal{E}$ and Hilbert space $X$, we define $\mathcal{L}_{2}^{\mathcal{E}}(X)$ as the subspace in $\mathcal{L}_{2}(X)$ of all elements $v$ such that the point value $v(\boldsymbol{y})$ (of a representative of $v$ ) is well-defined for all $\boldsymbol{y} \in \mathcal{E}$. In what folllows, $\mathcal{E}$ is fixed.

For $v \in \mathcal{L}_{2}^{\mathcal{E}}(X)$, we introduce the tensor product operator $\Delta_{s}^{I}$ for $s \in \mathbb{F}$ by

$$
\Delta_{s}^{I}(v):=\bigotimes_{j \in \mathbb{N}} \Delta_{s_{j}}^{I}(v)
$$

where the univariate operator $\Delta_{s_{j}}^{I}$ is applied to the univariate function $v$ by considering $v$ as a function of variable $y_{i}$ with the other variables held fixed. From the definition of $\mathcal{L}_{2}^{\mathcal{E}}(X)$ one can see that the operators $\Delta_{s}^{I}$ are well-defined for all $s \in \mathbb{F}$.

Next, we introduce the interpolation operator $I_{\Lambda}$ for a given finite set $\Lambda \subset \mathbb{F}$ by

$$
I_{\Lambda}:=\sum_{s \in \Lambda} \Delta_{s}^{I}
$$

Let Assumption 2.1 hold for Hilbert spaces $X^{1}$ and $X^{2}$. We introduce the interpolation operator $\mathcal{I}_{G}$ : $\mathcal{L}_{2}^{\mathcal{E}}\left(X^{2}\right) \rightarrow \mathcal{V}(G)$ for a given finite set $G \subset \mathbb{N}_{0} \times \mathbb{F}$ by

$$
\mathcal{I}_{G} v:=\sum_{(k, \boldsymbol{s}) \in G} \delta_{k} \Delta_{\boldsymbol{s}}^{I}(v)
$$

Notice that $\mathcal{I}_{G} v$ is a linear (non-adaptive) method of fully discrete polynomial interpolation approximation which is the sum taken over the indices set $G$, of mixed tensor products of dyadic scale successive differences of "spatial" approximations to $v$, and of successive differences of their parametric Lagrange interpolating polynomials. It has been introduced in [14] (see also [16]). A similar construction for the multi-index stochastic collocation method for computing the expected value of a functional of the solution to elliptic PDEs with random data has been introduced in $[21,22]$ by using Clenshaw-Curtis points for quadrature.

A set $\Lambda \subset \mathbb{F}$ is called downward closed if the inclusion $s \in \Lambda$ yields the inclusion $s^{\prime} \in \Lambda$ for every $s^{\prime} \in \mathbb{F}$ such that $s^{\prime} \leq s$. The inequality $s^{\prime} \leq s$ means that $s_{j}^{\prime} \leq s_{j}, j \in \mathbb{N}$. A sequence $\left(\sigma_{s}\right)_{s \in \mathbb{F}}$ is called increasing if $\sigma_{\boldsymbol{s}^{\prime}} \leq \sigma_{\boldsymbol{s}}$ for $\boldsymbol{s}^{\prime} \leq \boldsymbol{s}$. Put $R_{\boldsymbol{s}}:=\left\{\boldsymbol{s}^{\prime} \in \mathbb{F}: \boldsymbol{s}^{\prime} \leq \boldsymbol{s}\right\}$.

Theorem 3.8. Let $0<p \leq 2$. Let Assumption 2.1 hold for Hilbert spaces $X^{1}$ and $X^{2}$. Assume that $\left(Y_{m}\right)_{m \in \mathbb{N}_{0}}$ is a sequence satisfying the condition (3.15) for some positive numbers $\tau$ and $C$. Let $v \in \mathcal{L}_{2}^{\mathcal{E}}\left(X^{2}\right)$ be represented by the series (2.5). Assume that for $r=1,2$ there exist increasing sequences $\left(\sigma_{r ; s}\right)_{s \in \mathbb{F}}$ of numbers strictly larger than 1 such that

$$
\sum_{\boldsymbol{s} \in \mathbb{F}}\left(\sigma_{r ; \boldsymbol{s}}\left\|v_{\boldsymbol{s}}\right\|_{X^{r}}\right)^{2}<\infty
$$


and $\left(p_{\boldsymbol{s}}(2 \theta, \lambda) \sigma_{r ; \boldsymbol{s}}^{-1}\right)_{\boldsymbol{s} \in \mathbb{F}} \in \ell_{q_{r}}(\mathbb{F})$ for some $0<q_{1} \leq q_{2}<\infty$ with $q_{1}<2$, where

$$
\theta:=\tau+\varepsilon+5 / 4, \quad \lambda:=\max \left(C_{\infty, 2}, C_{2, \infty}, C_{\varepsilon}, 1\right),
$$

$C_{\infty, 2}, C_{2, \infty}$ are as in Lemma 3.1, $\varepsilon$ is arbitrary positive number and $C_{\varepsilon}$ is as in Lemma 3.7. For $\xi>0$, let $G(\xi)$ be the set defined as in (3.5).

Then for each $n \in \mathbb{N}$ there exists a number $\xi_{n}$ such that for the interpolation operator $\mathcal{I}_{G\left(\xi_{n}\right)}: \mathcal{L}_{2}^{\mathcal{E}}\left(X^{2}\right) \rightarrow$ $\mathcal{V}\left(G\left(\xi_{n}\right)\right)$, we have that $\operatorname{dim} \mathcal{V}\left(G\left(\xi_{n}\right)\right) \leq n$ and

$$
\left\|v-\mathcal{I}_{G\left(\xi_{n}\right)} v\right\|_{\mathcal{L}_{p}\left(X^{1}\right)} \leq C n^{-\min (\alpha, \beta)} .
$$

The rate $\alpha$ corresponds to the approximation of a single function in $X^{2}$ as given by (2.3). The rate $\beta$ is given by

$$
\beta:=\left(\frac{1}{q_{1}}-\frac{1}{2}\right) \frac{\alpha}{\alpha+\delta}, \quad \delta:=\frac{1}{q_{1}}-\frac{1}{q_{2}} .
$$

The constant $C$ in (3.19) is independent of $v$ and $n$.

Proof. Clearly, by the inequality $\|\cdot\|_{\mathcal{L}_{p}\left(X^{1}\right)} \leq\|\cdot\|_{\mathcal{L}_{2}\left(X^{1}\right)}$ it is sufficient to prove the theorem for $p=2$. By Lemmata 3.3 and 3.4 the series (2.5) and (3.4) converge absolutely, and therefore, unconditionally in the Hilbert space $\mathcal{L}_{2}\left(X^{1}\right)$ to $v$. We have that $\Delta_{s}^{I} H_{s^{\prime}}=0$ for every $s \not \leq s^{\prime}$. Moreover, if $\Lambda \subset \mathbb{F}$ is downward closed set, $I_{\Lambda} H_{s}=H_{s}$ for every $s \in \Lambda$, and hence we can write

$$
I_{\Lambda} v=I_{\Lambda}\left(\sum_{\boldsymbol{s} \in \mathbb{F}} v_{\boldsymbol{s}} H_{\boldsymbol{s}}\right)=\sum_{\boldsymbol{s} \in \mathbb{F}} v_{\boldsymbol{s}} I_{\Lambda} H_{\boldsymbol{s}}=\sum_{\boldsymbol{s} \in \Lambda} v_{\boldsymbol{s}} H_{\boldsymbol{s}}+\sum_{\boldsymbol{s} \notin \Lambda} v_{\boldsymbol{s}} I_{\Lambda \cap R_{\boldsymbol{s}}} H_{\boldsymbol{s}} .
$$

Let us first consider the case $\alpha \leq 1 / q_{2}-1 / 2$. Let $\xi>0$ be given. For $k \in \mathbb{N}_{0}$, put

$$
\Lambda_{k}:=\{s \in \mathbb{F}:(k, s) \in G(\xi)\}=\left\{s \in \mathbb{F}: \sigma_{2, s}^{q_{2}} \leq 2^{-k} \xi\right\} .
$$

Observe that $\Lambda_{k}=\emptyset$ for all $k>k^{*}:=\left\lfloor\log _{2} \xi\right\rfloor$, and consequently, we have that

$$
\mathcal{I}_{G(\xi)} v=\sum_{k=0}^{k^{*}} \delta_{k}\left(\sum_{s \in \Lambda_{k}} \Delta_{s}^{I}\right) v=\sum_{k=0}^{k^{*}} \delta_{k} I_{\Lambda_{k}} v
$$

Since the sequence $\left(\sigma_{2 ; \boldsymbol{s}}\right)_{\boldsymbol{s} \in \mathbb{F}}$ is increasing, $\Lambda_{k}$ are downward closed sets, and consequently, the sequence $\left\{\Lambda_{k}\right\}_{k=0}^{k^{*}}$ is nested in the inverse order, i.e., $\Lambda_{k^{\prime}} \subset \Lambda_{k}$ if $k^{\prime}>k$, and $\Lambda_{0}$ is the largest and $\Lambda_{k^{*}}=\left\{0_{\mathbb{F}}\right\}$. Therefore, from the unconditional convergence of the series (3.4) to $v,(3.22)$ and (3.21) we derive that

$$
\begin{aligned}
\mathcal{I}_{G(\xi)} v & =\sum_{k=0}^{k^{*}} \sum_{\boldsymbol{s} \in \Lambda_{k}} \delta_{k}\left(v_{\boldsymbol{s}}\right) H_{\boldsymbol{s}}+\sum_{k=0}^{k^{*}} \sum_{\boldsymbol{s} \notin \Lambda_{k}} \delta_{k}\left(v_{\boldsymbol{s}}\right) I_{\Lambda_{k} \cap R_{\boldsymbol{s}}} H_{\boldsymbol{s}} \\
& =\mathcal{S}_{G(\xi)} v+\sum_{k=0}^{k^{*}} \sum_{\boldsymbol{s} \notin \Lambda_{k}} \delta_{k}\left(v_{\boldsymbol{s}}\right) I_{\Lambda_{k} \cap R_{\boldsymbol{s}}} H_{\boldsymbol{s}} .
\end{aligned}
$$

This implies that

$$
v-\mathcal{I}_{G(\xi)} v=v-\mathcal{S}_{G(\xi)} v-\sum_{k=0}^{k^{*}} \sum_{\boldsymbol{s} \notin \Lambda_{k}} \delta_{k}\left(v_{\boldsymbol{s}}\right) I_{\Lambda_{k} \cap R_{\boldsymbol{s}}} H_{\boldsymbol{s}} .
$$

Hence,

$$
\left\|v-\mathcal{I}_{G(\xi)} v\right\|_{\mathcal{L}_{2}\left(X^{1}\right)} \leq\left\|v-\mathcal{S}_{G(\xi)} v\right\|_{\mathcal{L}_{2}\left(X^{1}\right)}+\sum_{(k, \boldsymbol{s}) \notin G(\xi)}\left\|\delta_{k}\left(v_{\boldsymbol{s}}\right)\right\|_{X^{1}}\left\|I_{\Lambda_{k} \cap R_{\boldsymbol{s}}} H_{\boldsymbol{s}}\right\|_{L_{2}\left(\mathbb{R}^{\infty}, \gamma\right)} .
$$


Lemma 3.5 gives

$$
\left\|v-\mathcal{S}_{G(\xi)} u\right\|_{\mathcal{L}_{2}\left(X^{1}\right)} \leq C \xi^{-\alpha}
$$

Let us estimate the sum in the right-hand side of (3.23). We have that

$$
\left\|I_{\Lambda_{k} \cap R_{s}} H_{\boldsymbol{s}}\right\|_{L_{2}\left(\mathbb{R}^{\infty}, \gamma\right)} \leq \sum_{\boldsymbol{s}^{\prime} \in \Lambda_{k} \cap R_{\boldsymbol{s}}}\left\|\Delta_{\boldsymbol{s}^{\prime}}^{I}\left(H_{\boldsymbol{s}}\right)\right\|_{L_{2}\left(\mathbb{R}^{\infty}, \gamma\right)} .
$$

We estimate the norm in the sum in the right-hand side. Assuming $s \in \mathbb{F}$ to be such that $\operatorname{supp}(s) \subset\{1, \ldots, J\}$, we have $\Delta_{s^{\prime}}^{I}\left(H_{s}\right)=\prod_{j=1}^{J} \Delta_{s_{j}^{\prime}}^{I}\left(H_{s_{j}}\right)$. Since $\Delta_{s_{j}^{\prime}}^{I}\left(H_{s_{j}}\right)$ is a polynomial of degree $\leq s_{j}^{\prime}$ in variable $y_{j}$, from Lemma 3.1 we obtain that

$$
\begin{aligned}
\left\|\Delta_{\boldsymbol{s}^{\prime}}^{I}\left(H_{\boldsymbol{s}}\right)\right\|_{L_{2}(\mathbb{R}, \gamma)} & =\prod_{j=1}^{J}\left\|\Delta_{s_{j}^{\prime}}^{I}\left(H_{s_{j}}\right)\right\|_{L_{2}(\mathbb{R}, \gamma)}=\prod_{j=1}^{J}\left\|\Delta_{s_{j}^{\prime}}^{I}\left(H_{s_{j}}\right) \sqrt{g}\right\|_{L_{2}(\mathbb{R})} \\
& \leq p_{\boldsymbol{s}^{\prime}}(\theta, \lambda) \prod_{j=1}^{J}\left\|\Delta_{s_{j}^{\prime}}^{I}\left(H_{s_{j}}\right) \sqrt{g}\right\|_{L_{\infty}(\mathbb{R})}
\end{aligned}
$$

where $\theta=1 / 4, \lambda:=C_{2, \infty}$ and $C_{2, \infty}$ is the constant in Lemma 3.1. Due to the assumption (3.15), we have by Lemmata 3.7 and 3.2 that

$$
\begin{aligned}
\left\|\Delta_{s_{j}^{\prime}}^{I}\left(H_{s_{j}}\right) \sqrt{g}\right\|_{L_{\infty}(\mathbb{R})} & \leq\left(1+C_{\varepsilon} s_{j}^{\prime}\right)^{\tau+\varepsilon}\left\|H_{s_{j}} \sqrt{g}\right\|_{L_{\infty}(\mathbb{R})} \\
& \leq\left(1+C_{\varepsilon} s_{j}^{\prime}\right)^{\tau+\varepsilon}\left(1+C_{\infty, 2,2} s_{j}\right)^{1 / 4}\left\|H_{s_{j}} \sqrt{g}\right\|_{L_{2}(\mathbb{R})} \\
& =\left(1+C_{\varepsilon} s_{j}^{\prime}\right)^{\tau+\varepsilon}\left(1+C_{\infty, 2,2} s_{j}\right)^{1 / 4}
\end{aligned}
$$

and consequently,

$$
\left\|\Delta_{\boldsymbol{s}^{\prime}}^{I}\left(H_{\boldsymbol{s}}\right)\right\|_{L_{2}\left(\mathbb{R}^{\infty}, \gamma\right)} \leq p_{\boldsymbol{s}^{\prime}}(\theta, \lambda) \leq p_{\boldsymbol{s}}(\theta, \lambda)
$$

where

$$
\theta:=\tau+\varepsilon+1 / 4, \quad \lambda:=\max \left(C_{\infty, 2}, C_{2, \infty}, C_{\varepsilon}\right) .
$$

Substituting $\left\|\Delta_{\boldsymbol{s}^{\prime}}^{I}\left(H_{\boldsymbol{s}}\right)\right\|_{L_{2}\left(\mathbb{R}^{\infty}, \gamma\right)}$ in (3.25) with the right-hand side of (3.26) gives that

$$
\left\|I_{\Lambda_{k} \cap R_{\boldsymbol{s}}} H_{\boldsymbol{s}}\right\|_{L_{2}\left(\mathbb{R}^{\infty}, \gamma\right)} \leq \sum_{\boldsymbol{s}^{\prime} \in \Lambda_{k} \cap R_{\boldsymbol{s}}} p_{\boldsymbol{s}}(\theta, \lambda) \leq\left|R_{\boldsymbol{s}}\right| p_{\boldsymbol{s}}(\theta, \lambda) \leq p_{\boldsymbol{s}}(1,1) p_{\boldsymbol{s}}(\theta, \lambda)
$$

where $\theta$ and $\lambda$ are as in (3.27). Hence,

$$
\left\|I_{\Lambda_{k} \cap R_{s}} H_{\boldsymbol{s}}\right\|_{L_{2}\left(\mathbb{R}^{\infty}, \gamma\right)} \leq p_{\boldsymbol{s}}(\theta, \lambda)
$$

where $\theta$ and $\lambda$ are as in (3.18). Denote by $\Sigma(\xi)$ the sum in the right-hand side of (3.23). By using (3.28) and (2.4) we estimate $\Sigma(\xi)$ as

$$
\begin{aligned}
\Sigma(\xi) & \leq C \sum_{(k, \boldsymbol{s}) \notin G(\xi)} 2^{-\alpha k} p_{\boldsymbol{s}}(\theta, \lambda)\left\|v_{\boldsymbol{s}}\right\|_{X^{2}}=C \sum_{\boldsymbol{s} \in \mathbb{F}} p_{\boldsymbol{s}}(\theta, \lambda)\left\|v_{\boldsymbol{s}}\right\|_{X^{2}} \sum_{2^{k}>\xi \sigma_{2 ; \boldsymbol{s}}^{-q_{2}}} 2^{-\alpha k} \\
& \leq C \sum_{\boldsymbol{s} \in \mathbb{F}} p_{\boldsymbol{s}}(\theta, \lambda)\left\|v_{\boldsymbol{s}}\right\|_{X^{2}}\left(\xi \sigma_{2 ; \boldsymbol{s}}^{-q_{2}}\right)^{-\alpha} \leq C \xi^{-\alpha} \sum_{\boldsymbol{s} \in \mathbb{F}} p_{\boldsymbol{s}}(\theta, \lambda) \sigma_{2 ; \boldsymbol{s}}^{q_{2} \alpha}\left\|v_{\boldsymbol{s}}\right\|_{X^{2}} .
\end{aligned}
$$


By the inequalities $2\left(1-q_{2} \alpha\right) \geq q_{2}$ and $\sigma_{2 ; s}>1$ and the assumptions we have that

$$
\begin{aligned}
\sum_{\boldsymbol{s} \in \mathbb{F}} p_{\boldsymbol{s}}(\theta, \lambda) \sigma_{2 ; \boldsymbol{s}}^{q_{2} \alpha}\left\|v_{\boldsymbol{s}}\right\|_{X^{2}} & \leq\left(\sum_{\boldsymbol{s} \in \mathbb{F}}\left(\sigma_{2 ; \boldsymbol{s}}\left\|v_{\boldsymbol{s}}\right\|_{X^{2}}\right)^{2}\right)^{1 / 2}\left(\sum_{\boldsymbol{s} \in \mathbb{F}} p_{\boldsymbol{s}}^{2}(\theta, \lambda) \sigma_{2 ; \boldsymbol{s}}^{-2\left(1-q_{2} \alpha\right)}\right)^{1 / 2} \\
& \leq\left(\sum_{\boldsymbol{s} \in \mathbb{F}}\left(\sigma_{2 ; \boldsymbol{s}}\left\|v_{\boldsymbol{s}}\right\|_{X^{2}}\right)^{2}\right)^{1 / 2}\left(\sum_{\boldsymbol{s} \in \mathbb{F}} p_{\boldsymbol{s}}(2 \theta, \lambda) \sigma_{2 ; \boldsymbol{s}}^{-q_{2}}\right)^{1 / 2}=C<\infty
\end{aligned}
$$

Thus, we obtain the estimate

$$
\Sigma(\xi):=\sum_{(k, \boldsymbol{s}) \notin G(\xi)}\left\|\delta_{k}\left(v_{\boldsymbol{s}}\right)\right\|_{X^{1}}\left\|I_{\Lambda_{k} \cap R_{s}} H_{\boldsymbol{s}}\right\|_{L_{2}\left(\mathbb{R}^{\infty}, \gamma\right)} \leq C \xi^{-\alpha}
$$

This together with (3.23) and (3.24) implies that

$$
\left\|v-\mathcal{I}_{G(\xi)} u\right\|_{\mathcal{L}_{2}\left(X^{1}\right)} \leq C \xi^{-\alpha} .
$$

Hence, similarly to (2.13)-(2.15), for each $n \in \mathbb{N}$ we can find a number $\xi_{n}$ such that $\operatorname{dim} \mathcal{V}\left(G\left(\xi_{n}\right)\right) \leq n$ and

$$
\left\|v-\mathcal{I}_{G\left(\xi_{n}\right)} v\right\|_{\mathcal{L}_{p}\left(X^{1}\right)} \leq C n^{-\alpha}, \quad \alpha \leq 1 / q_{2}-1 / 2 .
$$

We now consider the case $\alpha>1 / q_{2}-1 / 2$. Observe that the unconditional convergence of the series (2.5) and the uniform boundedness of the operators $P_{n}$ in $X^{1}$ imply that

$$
\delta_{k} \Delta_{\boldsymbol{s}}^{I} v=\Delta_{\boldsymbol{s}}^{I} \delta_{k} v=\sum_{\boldsymbol{s}^{\prime} \in \mathbb{F}} \delta_{k}\left(v_{\boldsymbol{s}^{\prime}}\right) \Delta_{\boldsymbol{s}}^{I}\left(H_{\boldsymbol{s}^{\prime}}\right)
$$

and

$$
P_{n} v=\sum_{\boldsymbol{s} \in \mathbb{F}} P_{n}\left(v_{\boldsymbol{s}}\right) H_{\boldsymbol{s}}
$$

with convergence of the series in $\mathcal{L}_{2}\left(X^{1}\right)$. Put $\Lambda(\xi):=\left\{s \in \mathbb{F}: \sigma_{1 ; s}^{q_{1}} \leq \xi\right\}$ and $B(\xi, s):=\left\{k \in \mathbb{N}_{0}: 2^{k} \leq\right.$ $\left.\sigma_{2 ; \boldsymbol{s}}^{-(\alpha+1 / 2)^{-1}} \xi^{\vartheta(\alpha+1 / 2)^{-1}}\right\}$ for $\xi>0$ with $\vartheta$ as in (3.6). By using of these equalities and the unconditional convergence of the series (2.5) and (3.4), with $N(\xi, s):=2^{\left\lfloor\log _{2}\left(\sigma_{2 ; s}^{-(\alpha+1 / 2)^{-1}} \xi^{\vartheta(\alpha+1 / 2)^{-1}}\right)\right\rfloor}$ we derive the equalities

$$
\begin{aligned}
\mathcal{I}_{G(\xi)} v & =\sum_{(k, \boldsymbol{s}) \in G(\xi)} \delta_{k} \Delta_{\boldsymbol{s}}^{I} v=\sum_{\boldsymbol{s} \in \Lambda(\xi)} \Delta_{\boldsymbol{s}}^{I}\left(\sum_{k \in B(\xi, \boldsymbol{s})} \delta_{k} v\right) \\
& =\sum_{\boldsymbol{s} \in \Lambda(\xi)} P_{N(\xi, \boldsymbol{s})}\left(v_{\boldsymbol{s}}\right) H_{\boldsymbol{s}}+\sum_{\boldsymbol{s} \notin \Lambda(\xi)} P_{N(\xi, \boldsymbol{s})}\left(v_{\boldsymbol{s}}\right) I_{\Lambda(\xi) \cap R_{\boldsymbol{s}}}\left(H_{\boldsymbol{s}}\right) \\
& =\sum_{\boldsymbol{s} \in \Lambda(\xi)}\left(\sum_{k \in B(\xi, \boldsymbol{s})} \delta_{k} v\right) H_{\boldsymbol{s}}+\sum_{\boldsymbol{s} \notin \Lambda(\xi)} P_{N(\xi, \boldsymbol{s})}\left(v_{\boldsymbol{s}}\right) I_{\Lambda(\xi) \cap R_{\boldsymbol{s}}}\left(H_{\boldsymbol{s}}\right) \\
& =\mathcal{S}_{G(\xi)} v+\sum_{\boldsymbol{s} \notin \Lambda(\xi)} P_{N(\xi, \boldsymbol{s})}\left(v_{\boldsymbol{s}}\right) I_{\Lambda(\xi) \cap R_{\boldsymbol{s}}}\left(H_{\boldsymbol{s}}\right) .
\end{aligned}
$$


Hence,

$$
\left\|v-\mathcal{I}_{G(\xi)} v\right\|_{\mathcal{L}_{2}\left(X^{1}\right)} \leq\left\|v-\mathcal{S}_{G(\xi)} v\right\|_{\mathcal{L}_{2}\left(X^{1}\right)}+\sum_{\boldsymbol{s} \notin \Lambda(\xi)}\left\|P_{N(\xi, s)}\left(v_{\boldsymbol{s}}\right)\right\|_{X^{1}}\left\|I_{\Lambda(\xi) \cap R_{\boldsymbol{s}}} H_{\boldsymbol{s}}\right\|_{L_{2}\left(\mathbb{R}^{\infty}, \gamma\right)} .
$$

Lemma 3.5 gives

$$
\left\|v-\mathcal{S}_{G(\xi)} v\right\|_{\mathcal{L}_{2}\left(X^{1}\right)} \leq C \xi^{-\left(1 / q_{1}-1 / 2\right)} .
$$

The sum in the right-hand side of (3.31) can be estimated by

$$
\sum_{\boldsymbol{s} \notin \Lambda(\xi)}\left\|P_{N(\xi, \boldsymbol{s})}\left(v_{\boldsymbol{s}}\right)\right\|_{X^{1}}\left\|I_{\Lambda(\xi) \cap R_{\boldsymbol{s}}} H_{\boldsymbol{s}}\right\|_{L_{2}\left(\mathbb{R}^{\infty}, \gamma\right)} \leq C \sum_{\boldsymbol{s} \notin \Lambda(\xi)}\left\|v_{\boldsymbol{s}}\right\|_{X^{1}}\left\|I_{\Lambda(\xi) \cap R_{\boldsymbol{s}}} H_{\boldsymbol{s}}\right\|_{L_{2}\left(\mathbb{R}^{\infty}, \gamma\right)} .
$$

Similarly to (3.28), we have

$$
\left\|I_{\Lambda(\xi) \cap R_{s}} H_{\boldsymbol{s}}\right\|_{L_{2}\left(\mathbb{R}^{\infty}, \gamma\right)} \leq p_{\boldsymbol{s}}(\theta, \lambda)
$$

with the same $\theta$ and $\lambda$ as in (3.18). This gives the estimate

$$
\sum_{\boldsymbol{s} \notin \Lambda(\xi)}\left\|P_{N(\xi, s)}\left(v_{\boldsymbol{s}}\right)\right\|_{X^{1}}\left\|I_{\Lambda(\xi) \cap R_{\boldsymbol{s}}} H_{\boldsymbol{s}}\right\|_{L_{2}\left(\mathbb{R}^{\infty}, \gamma\right)} \leq C \sum_{\boldsymbol{s} \notin \Lambda(\xi)}\left\|v_{\boldsymbol{s}}\right\|_{X^{1}} p_{\boldsymbol{s}}(\theta, \lambda) .
$$

We have by the Hölder inequality and the hypothesis of the theorem,

$$
\begin{aligned}
\sum_{\boldsymbol{s} \notin \Lambda(\xi)}\left\|v_{\boldsymbol{s}}\right\|_{X^{1}} p_{\boldsymbol{s}}(\theta, \lambda) & \leq\left(\sum_{\sigma_{1 ; \boldsymbol{s}}>\xi^{1 / q_{1}}}\left(\sigma_{1 ; \boldsymbol{s}}\left\|v_{\boldsymbol{s}}\right\|_{X^{1}}\right)^{2}\right)^{1 / 2}\left(\sum_{\sigma_{1 ; \boldsymbol{s}}>\xi^{1 / q_{1}}} p_{\boldsymbol{s}}^{2}(\theta, \lambda) \sigma_{1 ; \boldsymbol{s}}^{-2}\right)^{1 / 2} \\
& \leq C\left(\sum_{\sigma_{1 ; \boldsymbol{s}}>\xi^{1 / q_{1}}} p_{\boldsymbol{s}}^{2}(\theta, \lambda) \sigma_{1 ; \boldsymbol{s}}^{-q_{1}} \sigma_{1 ; \boldsymbol{s}}^{-\left(2-q_{1}\right)}\right)^{1 / 2} \\
& \leq C \xi^{-\left(1 / q_{1}-1 / 2\right)}\left(\sum_{\boldsymbol{s} \in \mathbb{F}} p_{\boldsymbol{s}}(2 \theta, \lambda) \sigma_{1 ; \boldsymbol{s}}^{-q_{1}}\right)^{1 / 2} \leq C \xi^{-\left(1 / q_{1}-1 / 2\right)}
\end{aligned}
$$

Combining (3.31)-(3.34) leads to the estimate

$$
\left\|v-\mathcal{I}_{G(\xi)} v\right\|_{\mathcal{L}_{2}\left(X^{1}\right)} \leq C \xi^{-\left(1 / q_{1}-1 / 2\right)}
$$

For the dimension of the space $\mathcal{V}(G(\xi))$, with $q:=(\alpha+1 / 2) q_{2}>1$ and $1 / q^{\prime}+1 / q=1$ we have that

$$
\begin{aligned}
\operatorname{dim} \mathcal{V}(G(\xi)) & \leq \sum_{(k, \boldsymbol{s}) \in G(\xi)} \operatorname{dim} V_{2^{k}} \leq \sum_{\sigma_{1 ; s}^{q_{1} \leq \xi}} \sum_{2^{(\alpha+1 / 2) k} \sigma_{2 ; s} \leq \xi^{\vartheta}} 2^{k} \\
& \leq 2 \sum_{\sigma_{1 ; s}^{q_{1} \leq \xi}} \xi^{\vartheta(\alpha+1 / 2)^{-1}} \sigma_{2 ; \boldsymbol{s}}^{-(\alpha+1 / 2)^{-1}} \leq 2 \xi^{\vartheta(\alpha+1 / 2)^{-1}}\left(\sum_{\sigma_{1 ; s}^{q_{1} \leq \xi}} \sigma_{2 ; \boldsymbol{s}}^{-q_{2}}\right)^{1 / q}\left(\sum_{\sigma_{1 ; s}^{q_{1} \leq \xi}} 1\right)^{1 / q^{\prime}} \\
& \leq 2 \xi^{\vartheta(\alpha+1 / 2)^{-1}}\left(\sum_{\boldsymbol{s} \in \mathbb{F}} \sigma_{2 ; \boldsymbol{s}}^{-q_{2}}\right)^{1 / q}\left(\sum_{\boldsymbol{s} \in \mathbb{F}} \xi \sigma_{1 ; \boldsymbol{s}}^{-q_{1}}\right)^{1 / q^{\prime}} \\
& =M \xi^{\vartheta(\alpha+1 / 2)^{-1}+1 / q^{\prime}}=M \xi^{1+\delta / \alpha},
\end{aligned}
$$


where $M:=2\left\|\left(\sigma_{2 ; s}^{-1}\right)\right\|_{\ell_{q_{2}}(\mathbb{F})}^{q_{2} / q}\left\|\left(\sigma_{1 ; s}^{-1}\right)\right\|_{\ell_{q_{1}}(\mathbb{F})}^{q_{1} / q^{\prime}}$. For any $n \in \mathbb{N}$, letting $\xi_{n}$ be a number satisfying the inequalities

$$
M \xi_{n}^{1+\delta / \alpha} \leq n<2 M \xi_{n}^{1+\delta / \alpha},
$$

we derive that $\operatorname{dim} \mathcal{V}\left(G\left(\xi_{n}\right)\right) \leq n$. On the other hand, by (3.36),

$$
\xi_{n}^{-\left(1 / q_{1}-1 / 2\right)} \leq(2 M)^{\frac{\alpha}{\alpha+\delta}} n^{-\left(1 / q_{1}-1 / 2\right) \frac{\alpha}{\alpha+\delta}} .
$$

This together with (3.35) proves that

$$
\left\|v-\mathcal{I}_{G\left(\xi_{n}\right)} v\right\|_{\mathcal{L}_{p}\left(X^{1}\right)} \leq C n^{-\beta}, \quad \alpha>1 / q_{2}-1 / 2 .
$$

By combining the last estimate and (3.29) we derive (3.19).

Denote by $\Gamma_{s}$ and $\Gamma(\Lambda)$ the set of interpolation points in the operators $\Delta_{s}^{I}$ and $I_{\Lambda}$, respectively. We have that $\Gamma_{\boldsymbol{s}}=\left\{\boldsymbol{y}_{\boldsymbol{s}-\boldsymbol{e} ; \boldsymbol{m}}: e \in \mathbb{E}_{\boldsymbol{s}} ; m_{j}=0, \ldots, s_{j}-e_{j}, j \in \mathbb{N}\right\}$ and $\Gamma(\Lambda)=\cup_{\boldsymbol{s} \in \Lambda} \Gamma_{\boldsymbol{s}}$, where $\mathbb{E}_{\boldsymbol{s}}$ is the subset in $\mathbb{F}$ of all $\boldsymbol{e}$ such that $e_{j}$ is 1 or 0 if $s_{j}>0$, and $e_{j}$ is 0 if $s_{j}=0$, and $\boldsymbol{y}_{\boldsymbol{s} ; \boldsymbol{m}}:=\left(y_{s_{j} ; m_{j}}\right)_{j \in \mathbb{N}}$.

Remark 3.9. (i) Observe that the operator $\mathcal{I}_{G\left(\xi_{n}\right)}$ in Theorem 3.8 can be represented in the form of a multilevel approximation method with $k_{n}$ levels:

$$
\mathcal{I}_{G\left(\xi_{n}\right)}=\sum_{k=0}^{k_{n}} \delta_{k} I_{\Lambda_{k}\left(\xi_{n}\right)}
$$

where $k_{n}:=\left\lfloor\log _{2} \xi_{n}\right\rfloor$ and for $k \in \mathbb{N}_{0}$ and $\xi>0$,

$$
\Lambda_{k}(\xi):= \begin{cases}\left\{s \in \mathbb{F}: \sigma_{2 ; s}^{q_{2}} \leq 2^{-k} \xi\right\} & \text { if } \quad \alpha \leq 1 / q_{2}-1 / 2 \\ \left\{\boldsymbol{s} \in \mathbb{F}: \sigma_{1 ; s}^{q_{1}} \leq \xi, \sigma_{2 ; s} \leq 2^{-(\alpha+1 / 2) k} \xi^{\vartheta}\right\} & \text { if } \quad \alpha>1 / q_{2}-1 / 2 .\end{cases}
$$

Moreover, $\Lambda_{k}\left(\xi_{n}\right)$ are downward closed sets, and consequently, the sequence $\left\{\Lambda_{k}\left(\xi_{n}\right)\right\}_{k=0}^{k_{n}}$ is nested in the inverse order, i.e., $\Lambda_{k^{\prime}}\left(\xi_{n}\right) \subset \Lambda_{k}\left(\xi_{n}\right)$ if $k^{\prime}>k$, and $\Lambda_{0}\left(\xi_{n}\right)$ is the largest and $\Lambda_{k_{n}}\left(\xi_{n}\right)=\left\{0_{\mathbb{F}}\right\}$.

(ii) Theorem 3.8 is a non-adaptive "collocation" extension of Theorem 3.1 in [1] for the lognormal case. The approximant $\mathcal{I}_{G\left(\xi_{n}\right)} v$ belongs to the space $\mathcal{V}\left(G\left(\xi_{n}\right)\right) \subset \mathcal{V}\left(X^{2}\right)$. The convergence rate $\min (\alpha, \beta)$ of the approximation by $\mathcal{I}_{G\left(\xi_{n}\right)} v$ is given in terms of $n$ where the thresholding parameter $\xi_{n}$ is chosen such that $\operatorname{dim}\left(\mathcal{V}\left(G\left(\xi_{n}\right)\right) \leq n\right.$. This rate is the same as the rate of the approximation by the truncated Hermite gpc expansion $\mathcal{S}_{G\left(\xi_{n}\right)} v$. The fully discrete polynomial interpolation approximation of $v \in \mathcal{L}_{2}^{\mathcal{E}}\left(X^{2}\right)$ by operators $\mathcal{I}_{G\left(\xi_{n}\right)}$ is based on the finite point-wise information in $\boldsymbol{y}$, more precisely, on $\left|\Gamma\left(\Lambda_{0}\left(\xi_{n}\right)\right)\right|$ of particular values of $v$ at the interpolation points $\boldsymbol{y} \in \Gamma\left(\Lambda_{0}\left(\xi_{n}\right)\right)$ and the approximations of $v(\boldsymbol{y}), \boldsymbol{y} \in \Gamma\left(\Lambda_{0}\left(\xi_{n}\right)\right)$, by $P_{2^{k}} v(\boldsymbol{y})$ for $k=0, \ldots, k_{n}$. Moreover, we have that

$$
\left|\Gamma\left(\Lambda_{0}(n)\right)\right| \leq \sum_{\boldsymbol{s} \in \Lambda_{0}(n)} p_{\boldsymbol{s}}(1,2)=\mathcal{O}(n)
$$

(iii) Under the assumptions of Theorem 3.8, by (3.30) we have that for every $v \in \mathcal{L}_{2}\left(X^{2}\right)$ and every $G \subset \mathbb{N}_{0} \times \mathbb{F}$,

$$
\mathcal{I}_{G} v=\sum_{(k, s) \in G} \Delta_{s}^{I}\left(\delta_{k} v\right)
$$


Theorem 3.10. Let $0<p \leq 2$. Let Assumption 2.1 hold for Hilbert spaces $X^{1}$ and $X^{2}$. Let $v \in \mathcal{L}_{2}^{\mathcal{E}}\left(X^{2}\right)$ be represented by the series (3.10). Assume that $\left(Y_{m}\right)_{m \in \mathbb{N}_{0}}$ is a sequence satisfying the condition (3.15) for some positive numbers $\tau$ and $C$. Assume that for $r=1,2$ there exist increasing sequences $\left(\sigma_{r ; s}\right)_{s \in \mathbb{F}_{\mathrm{ev}}}$ of numbers strictly larger than 1 such that

$$
\sum_{\boldsymbol{s} \in \mathbb{F}_{\mathrm{ev}}}\left(\sigma_{r ; \boldsymbol{s}}\left\|v_{\boldsymbol{s}}\right\|_{X^{r}}\right)^{2}<\infty
$$

and $\left(p_{\boldsymbol{s}}(2 \theta, \lambda) \sigma_{r ; \boldsymbol{s}}^{-1}\right)_{\boldsymbol{s} \in \mathbb{F}_{\mathrm{ev}}} \in \ell_{q_{r}}\left(\mathbb{F}_{\mathrm{ev}}\right)$ for some $0<q_{1} \leq q_{2}<\infty$ with $q_{1}<2$, where $\theta$ and $\lambda$ are as in (3.18). For $\xi>0$, let $G_{\mathrm{ev}}(\xi)$ be the set defined as in (3.11).

Then for each $n \in \mathbb{N}$ there exists a number $\xi_{n}$ such that for the interpolation operator $\mathcal{I}_{G_{\mathrm{ev}}\left(\xi_{n}\right)}: \mathcal{L}_{2}^{\mathcal{E}}\left(X^{2}\right) \rightarrow$ $\mathcal{V}\left(G_{\mathrm{ev}}\left(\xi_{n}\right)\right)$, we have that $\operatorname{dim} \mathcal{V}\left(G_{\mathrm{ev}}\left(\xi_{n}\right)\right) \leq n$ and

$$
\left\|v-\mathcal{I}_{G_{\mathrm{ev}}\left(\xi_{n}\right)} v\right\|_{\mathcal{L}_{p}\left(X^{1}\right)} \leq C n^{-\min (\alpha, \beta)} .
$$

The rate $\alpha$ corresponds to the approximation of a single function in $X^{2}$ as given by (2.3). The rate $\beta$ is given by (3.20). The constant $C$ in (3.37) is independent of $v$ and $n$.

Proof. The proof of this theorem is similar to the proof of Theorem 3.8 with some modification. For example, all the indices sets are taken from the sets $\mathbb{F}_{\text {ev }}$ and $\mathbb{N}_{0} \times \mathbb{F}_{\text {ev }}$ instead $\mathbb{F}$ and $\mathbb{N}_{0} \times \mathbb{F}$; estimates similar to (3.24) and (3.32) are given by Lemma 3.6 instead Lemma 3.5.

Corollary 3.11. Let $0<p \leq 2$. Let $v \in \mathcal{L}_{2}^{\mathcal{E}}(X)$ be represented by the series (2.2) for a Hilbert space $X$. Assume that $\left(Y_{m}\right)_{m \in \mathbb{N}_{0}}$ is a sequence satisfying the condition (3.15) for some positive numbers $\tau$ and $C$. Assume that there exists an increasing sequence $\left(\sigma_{\boldsymbol{s}}\right)_{\boldsymbol{s} \in \mathbb{F}}$ of numbers strictly larger than 1 such that

$$
\sum_{\boldsymbol{s} \in \mathbb{F}}\left(\sigma_{\boldsymbol{s}}\left\|v_{\boldsymbol{s}}\right\|_{X}\right)^{2}<\infty
$$

and $\left(p_{\boldsymbol{s}}(2 \theta, \max (2, \lambda)) \sigma_{\boldsymbol{s}}^{-1}\right)_{\boldsymbol{s} \in \mathbb{F}} \in \ell_{q}(\mathbb{F})$ for some $0<q<2$, where $\theta$ and $\lambda$ are as in (3.18). For $\xi>0$, define

$$
\Lambda(\xi):=\left\{s \in \mathbb{F}: \sigma_{s}^{q} \leq \xi\right\} .
$$

Then for each $n \in \mathbb{N}$ there exists a number $\xi_{n}$ such that $\left|\Gamma\left(\Lambda\left(\xi_{n}\right)\right)\right| \leq n$ and

$$
\left\|v-I_{\Lambda\left(\xi_{n}\right)} v\right\|_{\mathcal{L}_{p}(X)} \leq C n^{-(1 / q-1 / 2)} .
$$

The constant $C$ in (3.39) is independent of $v$ and $n$.

Proof. Similarly to the proof of Theorem 3.8 it is sufficient to prove (3.39) for $p=2$. In the same way as in proving (3.31), we can show that

$$
\left\|v-I_{\Lambda(\xi)} v\right\|_{\mathcal{L}_{2}\left(X^{1}\right)} \leq\left\|v-S_{\Lambda(\xi)} v\right\|_{\mathcal{L}_{2}\left(X^{1}\right)}+\sum_{\boldsymbol{s} \notin \Lambda(\xi)}\left\|v_{\boldsymbol{s}}\right\|_{X^{1}}\left\|I_{\Lambda(\xi) \cap R_{\boldsymbol{s}}} H_{\boldsymbol{s}}\right\|_{L_{2}\left(\mathbb{R}^{\infty}, \gamma\right)},
$$

where

$$
S_{\Lambda(\xi)} v:=\sum_{s \in \Lambda(\xi)} v_{\boldsymbol{s}} H_{\boldsymbol{s}}
$$

By estimating $\left\|v-S_{\Lambda(\xi)} v\right\|_{\mathcal{L}_{2}\left(X^{1}\right)}$ and $\sum_{\boldsymbol{s} \notin \Lambda(\xi)}\left\|v_{\boldsymbol{s}}\right\|_{X^{1}}\left\|I_{\Lambda(\xi) \cap R_{s}} H_{\boldsymbol{s}}\right\|_{L_{2}\left(\mathbb{R}^{\infty}, \gamma\right)}$ similarly to (2.16), (3.33) and (3.34), respectively, we derive

$$
\left\|v-I_{\Lambda(\xi)} v\right\|_{\mathcal{L}_{2}\left(X^{1}\right)} \leq C \xi^{-(1 / q-1 / 2)} .
$$


Since $\left|\Gamma_{\boldsymbol{s}}\right| \leq \prod_{j \in \mathbb{N}}\left(2 s_{j}+1\right)=p_{\boldsymbol{s}}(1,2)$, we have from the definition

$$
|\Gamma(\Lambda(\xi))| \leq \sum_{\boldsymbol{s} \in \Lambda(\xi)}\left|\Gamma_{\boldsymbol{s}}\right| \leq \sum_{\xi \sigma_{\boldsymbol{s}}^{-q} \geq 1} p_{\boldsymbol{s}}(1,2) \leq M \xi
$$

where $M:=\sum_{\boldsymbol{s} \in \mathbb{F}} p_{\boldsymbol{s}}(1,2) \sigma_{\boldsymbol{s}}^{-q}<\infty$ by the assumption. For any $n \in \mathbb{N}$, by choosing a number $\xi_{n}$ satisfying the inequalities $M \xi_{n} \leq n<2 M \xi_{n}$, we derive (3.39).

Similarly to Corollary 3.11 we have the following

Corollary 3.12. Let $v \in \mathcal{L}_{2}^{\mathcal{E}}(X)$ be represented by the series (3.10) for a Hilbert space $X$. Assume that $\left(Y_{m}\right)_{m \in \mathbb{N}_{0}}$ is a sequence satisfying the condition (3.15) for some positive numbers $\tau$ and $C$. Assume that there exists an increasing sequence $\left(\sigma_{\boldsymbol{s}}\right)_{\boldsymbol{s} \in \mathbb{F}_{\mathrm{ev}}}$ of numbers strictly larger than 1 such that

$$
\sum_{\boldsymbol{s} \in \mathbb{F}_{\mathrm{ev}}}\left(\sigma_{\boldsymbol{s}}\left\|v_{\boldsymbol{s}}\right\|_{X}\right)^{2}<\infty
$$

and $\left(p_{\boldsymbol{s}}(2 \theta, \max (2, \lambda)) \sigma_{\boldsymbol{s}}^{-1}\right)_{\boldsymbol{s} \in \mathbb{F}_{\mathrm{ev}}} \in \ell_{q}\left(\mathbb{F}_{\mathrm{ev}}\right)$ for some $0<q<2$, where $\theta$ and $\lambda$ are as in (3.18). For $\xi>0$, define

$$
\Lambda_{\mathrm{ev}}(\xi):=\Lambda(\xi) \cap \mathbb{F}_{\mathrm{ev}}=\left\{s \in \mathbb{F}_{\mathrm{ev}}: \sigma_{s}^{q} \leq \xi\right\} .
$$

Then for each $m \in \mathbb{N}$ there exists a number $\xi_{n}$ such that $\left|\Gamma\left(\Lambda_{\mathrm{ev}}\left(\xi_{n}\right)\right)\right| \leq n$ and

$$
\left\|v-I_{\Lambda_{\mathrm{ev}}\left(\xi_{n}\right)} v\right\|_{\mathcal{L}_{p}(X)} \leq C n^{-(1 / q-1 / 2)} .
$$

The constant $C$ in (3.42) is independent of $v$ and $n$.

Remark 3.13. (i) Theorem 3.10 and Corollary 3.12 will be applied in proving the convergence rates of fully and non-fully discrete integration in the next section.

(ii) The bound $\left\|v-I_{\Lambda_{n}} v\right\|_{L_{2}\left(\mathbb{R}^{\infty}, \mathcal{H}, \gamma\right)} \leq C n^{-(1 / q-1 / 2)}$ has been obtained in Theorem 3.14 of [18] for a Hilbert space $\mathcal{H}$, where $\Lambda_{n}$ is the set of $s$ corresponding to the $n$ largest elements of an $\ell_{q}$-summable majorant of the sequence $\left(\sigma_{s}^{-1} p_{\boldsymbol{s}}(\theta, \lambda)\right)_{\boldsymbol{s} \in \mathbb{F}}$.

(iii) The operators $I_{\Lambda(\xi)}$ and $I_{\Lambda_{\mathrm{ev}}(\xi)}$ represent non-adaptive collocation methods of approximation of $v \in X^{1}$ based on the particular values $v(\boldsymbol{y})$ at the points $\boldsymbol{y}$ in the grids $\Gamma(\Lambda(\xi))$ and $\Gamma\left(\Lambda_{\mathrm{ev}}(\xi)\right.$ ), respectively. Moreover, the sparsity of $\Gamma\left(\Lambda_{\mathrm{ev}}(\xi)\right)$ is much higher than that of $\Gamma(\Lambda(\xi))$ : the generating set $\Lambda_{\mathrm{ev}}(\xi)$ contains only even indices of $\Lambda(\xi)$. This remarkable property, in particular, plays an important role in improving the rate of quadrature of the solution to the parametrized elliptic PDEs with lognormal inputs (1.1), see Corollary 5.11 and its proof as well as Remark 5.12.

\section{INTEGRATION}

In this section, we construct general linear fully discrete methods for integration of functions taking values in $X^{2}$ and having a weighted $\ell_{2}$-summability of Hermite expansion coefficients for Hilbert spaces $X^{1}$ and $X^{2}$ satisfying a certain "spatial" approximation property, and their bounded linear functionals. In particular, we give convergence rates for these methods of integration which are derived from results on convergence rate of polynomial interpolation approximation in $\mathcal{V}_{1}\left(X^{1}\right)$ in Theorem 3.10. We also briefly consider linear non-fully discrete methods for integration.

If $v$ is a function defined on $\mathbb{R}$ taking values in a Hilbert space $X$, the function $I_{m}(v)$ in $(3.14)$ generates the quadrature formula defined as

$$
Q_{m}(v):=\int_{\mathbb{R}} I_{m}(v)(y) \mathrm{d} \gamma(y)=\sum_{k=0}^{m} \omega_{m ; k} v\left(y_{m ; k}\right),
$$


where

$$
\omega_{m ; k}:=\int_{\mathbb{R}} \ell_{m ; k}(y) \mathrm{d} \gamma(y)
$$

Notice that

$$
Q_{m}(\varphi)=\int_{\mathbb{R}} \varphi(y) \mathrm{d} \gamma(y)
$$

for every polynomial $\varphi$ of degree $\leq m$, due to the identity $I_{m}(\varphi)=\varphi$.

For integration purpose, we additionally assume that the sequence $Y_{m}$ as in (3.13) is symmetric for every $m \in \mathbb{N}_{0}$, i.e., $y_{m ; m-k}=y_{m ; k}$ for $k=0, \ldots, m$. The sequences $Y_{m}^{*}$ of the of the roots of the Hermite polynomials $H_{m+1}$ and their modifications $\bar{Y}_{m}^{*}$ are symmetric. Also, for the sequence $Y_{m}^{*}$, it is well-known that

$$
\omega_{m ; k}=\frac{1}{(m+1) H_{m}^{2}\left(y_{m ; k}^{*}\right)} .
$$

For a given sequence $\left(Y_{m}\right)_{m=0}^{\infty}$, we define the univariate operator $\Delta_{m}^{Q}$ for $m \in \mathbb{N}_{0}$ by

$$
\Delta_{m}^{Q}:=Q_{m}-Q_{m-1}
$$

with the convention $Q_{-1}:=0$.

For a function $v \in \mathcal{L}_{2}^{\mathcal{E}}(X)$, we introduce the interpolation operator $\Delta_{s}^{Q}$ defined for $s \in \mathbb{F}$ by

$$
\Delta_{s}^{Q}(v):=\bigotimes_{j \in \mathbb{N}} \Delta_{s_{j}}^{Q}(v)
$$

where the univariate operator $\Delta_{s_{j}}^{Q}$ is applied to the univariate function $v$ by considering $v$ as a function of variable $y_{i}$ with the other variables held fixed. As $\Delta_{s}^{I}$, the operators $\Delta_{s}^{Q}$ are well-defined for all $s \in \mathbb{F}$. For a finite set $\Lambda \subset \mathbb{F}$, we introduce the quadrature operator $Q_{\Lambda}$ which is generated by the interpolation operator $I_{\Lambda}$ as follows

$$
Q_{\Lambda} v:=\sum_{\boldsymbol{s} \in \Lambda} \Delta_{\boldsymbol{s}}^{Q}(v)=\int_{\mathbb{R}^{\infty}} I_{\Lambda} v(\boldsymbol{y}) \mathrm{d} \gamma(\boldsymbol{y})
$$

Further, if $\phi \in X^{\prime}$ is a bounded linear functional on $X$, denote by $\langle\phi, v\rangle$ the value of $\phi$ in $v$. For a finite set $\Lambda \subset \mathbb{F}$, the quadrature formula $Q_{\Lambda} v$ generates the quadrature formula $Q_{\Lambda}\langle\phi, v\rangle$ for integration of $\langle\phi, v\rangle$ by

$$
Q_{\Lambda}\langle\phi, v\rangle:=\left\langle\phi, Q_{\Lambda}\right\rangle=\int_{\mathbb{R}^{\infty}}\left\langle\phi, I_{\Lambda} v(\boldsymbol{y})\right\rangle \mathrm{d} \gamma(\boldsymbol{y}) .
$$

Let Assumption 2.1 hold for Hilbert spaces $X^{1}$ and $X^{2}$, and $v \in \mathcal{L}_{2}^{\mathcal{E}}\left(X^{2}\right)$. For a finite set $G \subset \mathbb{N}_{0} \times \mathbb{F}$, we introduce the quadrature operator $\mathcal{Q}_{G}$ which is generated by the interpolation operator $\mathcal{I}_{G}: \mathcal{L}_{2}^{\mathcal{E}}\left(X^{2}\right) \rightarrow \mathcal{V}(G)$, and which is defined for $v$ by

$$
\mathcal{Q}_{G} v:=\sum_{(k, \boldsymbol{s}) \in G} \delta_{k} \Delta_{\boldsymbol{s}}^{Q}(v)=\int_{\mathbb{R}^{\infty}} \mathcal{I}_{G} v(\boldsymbol{y}) \mathrm{d} \gamma(\boldsymbol{y}) .
$$

Further, if $\phi \in\left(X^{1}\right)^{\prime}$ is a bounded linear functional on $X^{1}$, for a finite set $G \subset \mathbb{N}_{0} \times \mathbb{F}$, the quadrature formula $\mathcal{Q}_{G} v$ generates the quadrature formula $\mathcal{Q}_{G}\langle\phi, v\rangle$ for integration of $\langle\phi, v\rangle$ by

$$
\mathcal{Q}_{G}\langle\phi, v\rangle:=\left\langle\phi, \mathcal{Q}_{G} v\right\rangle=\int_{\mathbb{R}^{\infty}}\left\langle\phi, \mathcal{I}_{G} v(\boldsymbol{y})\right\rangle \mathrm{d} \gamma(\boldsymbol{y}) .
$$

For a function $v \in \mathcal{L}_{2}^{\mathcal{E}}(X)$ and is represented by the series $(2.2)$, consider the function $v_{\text {ev }} \in \mathcal{L}_{2}^{\mathcal{E}}(X)$ defined by

$$
v_{\mathrm{ev}}:=\sum_{\boldsymbol{s} \in \mathbb{F}_{\mathrm{ev}}} v_{\boldsymbol{s}} H_{\boldsymbol{s}}
$$


From the obvious equality $\int_{\mathbb{R}} v(y) \mathrm{d} \gamma(y)=0$ for every odd function $v$, we have that

$$
\int_{\mathbb{R}^{\infty}} H_{\boldsymbol{s}}(\boldsymbol{y}) \mathrm{d} \gamma(\boldsymbol{y})=0, s \notin \mathbb{F}_{\mathrm{ev}},
$$

and hence,

$$
\int_{\mathbb{R}^{\infty}} v(\boldsymbol{y}) \mathrm{d} \gamma(\boldsymbol{y})=\int_{\mathbb{R}^{\infty}} v_{\mathrm{ev}}(\boldsymbol{y}) \mathrm{d} \gamma(\boldsymbol{y}) .
$$

Moreover, if $Y_{m}$ is symmetric for every $m \in \mathbb{N}_{0}$,

$$
\Delta_{\boldsymbol{s}^{\prime}}^{Q} H_{\boldsymbol{s}}(\boldsymbol{y})=0, \quad \boldsymbol{s} \notin \mathbb{F}_{\mathrm{ev}}, \boldsymbol{s}^{\prime} \in \mathbb{F} .
$$

The equalities (4.2) and (4.3) allow us to remove redundant non-even parametric points in the grid $\Gamma(\Lambda)$ for polynomial interpolation and use instead it the grid $\Gamma\left(\Lambda_{\mathrm{ev}}\right)$ with higher sparsity for constructing a quadrature formula, where $\Lambda_{\mathrm{ev}}:=\Lambda \cap \mathbb{F}_{\mathrm{ev}}$, see Theorem 4.1 and Corollary 4.2 and their proofs below. This reduces the cost of computation in numerical implementation.

Theorem 4.1. Under the hypothesis of Theorem 3.8, assume additionally that the sequences $Y_{m}, m \in \mathbb{N}_{0}$, are symmetric. For $\xi>0$, let $G_{\mathrm{ev}}(\xi)$ be the set defined as in (3.11). Then for the quadrature operator $\mathcal{Q}_{G_{\mathrm{ev}}(\xi)}$ generated by the interpolation operator $\mathcal{I}_{G_{\mathrm{ev}}(\xi)}: \mathcal{L}_{2}^{\mathcal{E}}\left(X^{2}\right) \rightarrow \mathcal{V}\left(G_{\mathrm{ev}}(\xi)\right)$, we have the following.

(i) For each $n \in \mathbb{N}$ there exists a number $\xi_{n}$ such that $\operatorname{dim} \mathcal{V}\left(G_{\mathrm{ev}}\left(\xi_{n}\right)\right) \leq n$ and

$$
\left\|\int_{\mathbb{R}^{\infty}} v(\boldsymbol{y}) \mathrm{d} \gamma(\boldsymbol{y})-\mathcal{Q}_{G_{\mathrm{ev}}\left(\xi_{n}\right)} v\right\|_{X^{1}} \leq C n^{-\min (\alpha, \beta)} .
$$

(ii) Let $\phi \in\left(X^{1}\right)^{\prime}$ be a bounded linear functional on $X^{1}$. Then for each $n \in \mathbb{N}$ there exists a number $\xi_{n}$ such that $\operatorname{dim} \mathcal{V}\left(G_{\mathrm{ev}}\left(\xi_{n}\right)\right) \leq n$ and

$$
\left|\int_{\mathbb{R}^{\infty}}\langle\phi, v(\boldsymbol{y})\rangle \mathrm{d} \gamma(\boldsymbol{y})-\mathcal{Q}_{G_{\mathrm{ev}}\left(\xi_{n}\right)}\langle\phi, v\rangle\right| \leq C n^{-\min (\alpha, \beta)} .
$$

The rate $\alpha$ corresponds to the approximation of a single function in $X^{2}$ as given by (2.3). The rate $\beta$ is given by (3.20). The constants $C$ in (4.4) and (4.5) are independent of $v$ and $n$.

Proof. For a given $n \in \mathbb{N}$, we approximate the integral in the right-hand side of (4.2) by $\mathcal{Q}_{G_{\mathrm{ev}}\left(\xi_{n}\right)}$ where $\xi_{n}$ is as in Theorem 3.10. By Lemmata 3.3 and 3.4 the series (2.5) and (3.4) converge absolutely, and therefore, unconditionally in the Hilbert space $\mathcal{L}_{2}\left(X^{1}\right)$ to $v$. Hence, by $(4.3)$ we derive that $\mathcal{Q}_{G_{\mathrm{ev}}\left(\xi_{n}\right)} v=\mathcal{Q}_{G_{\mathrm{ev}}\left(\xi_{n}\right)} v_{\mathrm{ev}}$. Due to (4.1) and (4.2) there holds the equality

$$
\int_{\mathbb{R}^{\infty}} v(\boldsymbol{y}) \mathrm{d} \gamma(\boldsymbol{y})-\mathcal{Q}_{G_{\mathrm{ev}}\left(\xi_{n}\right)} v=\int_{\mathbb{R}^{\infty}}\left(v_{\mathrm{ev}}(\boldsymbol{y})-\mathcal{I}_{G_{\mathrm{ev}}\left(\xi_{n}\right)} v_{\mathrm{ev}}(\boldsymbol{y})\right) \mathrm{d} \gamma(\boldsymbol{y}) .
$$

Hence, applying (3.37) in Theorem 3.10 for $p=1$, we obtain (i):

$$
\left\|\int_{\mathbb{R}^{\infty}} v(\boldsymbol{y}) \mathrm{d} \gamma(\boldsymbol{y})-\mathcal{Q}_{G_{\mathrm{ev}}\left(\xi_{n}\right)} v\right\|_{X^{1}} \leq\left\|v_{\mathrm{ev}}-\mathcal{I}_{G_{\mathrm{ev}}\left(\xi_{n}\right)} v_{\mathrm{ev}}\right\|_{\mathcal{L}_{1}\left(X^{1}\right)} \leq C n^{-\min (\alpha, \beta)} .
$$

For a given $n \in \mathbb{N}$, we approximate the integral $\int_{\mathbb{R}^{\infty}}\langle\phi, v(\boldsymbol{y})\rangle \mathrm{d} \gamma(\boldsymbol{y})$ by $\mathcal{Q}_{\Lambda_{\mathrm{ev}}\left(\xi_{n}\right)}\langle\phi, v\rangle$ where $\xi_{n}$ is as in Corollary 3.12. Similarly to (4.6), there holds the equality

$$
\int_{\mathbb{R}^{\infty}}\left\langle\phi, v_{\mathrm{ev}}(\boldsymbol{y})\right\rangle \mathrm{d} \gamma(\boldsymbol{y})-\mathcal{Q}_{G_{\mathrm{ev}}\left(\xi_{n}\right)}\left\langle\phi, v_{\mathrm{ev}}(\boldsymbol{y})\right\rangle=\int_{\mathbb{R}^{\infty}}\left\langle\phi, v_{\mathrm{ev}}(\boldsymbol{y})-\mathcal{I}_{G_{\mathrm{ev}}\left(\xi_{n}\right)} v_{\mathrm{ev}}(\boldsymbol{y})\right\rangle \mathrm{d} \gamma(\boldsymbol{y}) .
$$


Hence, applying (3.37) in Theorem 3.10 for $p=1$, we prove (ii):

$$
\begin{aligned}
\left|\int_{\mathbb{R}^{\infty}}\left\langle\phi, v_{\mathrm{ev}}(\boldsymbol{y})\right\rangle \mathrm{d} \gamma(\boldsymbol{y})-\mathcal{Q}_{G_{\mathrm{ev}}\left(\xi_{n}\right)}\langle\phi, v\rangle\right| & \leq \int_{\mathbb{R}^{\infty}}\left|\left\langle\phi, v_{\mathrm{ev}}(\boldsymbol{y})-\mathcal{I}_{G_{\mathrm{ev}}\left(\xi_{n}\right)} v_{\mathrm{ev}}(\boldsymbol{y})\right\rangle\right| \mathrm{d} \gamma(\boldsymbol{y}) \\
& \leq \int_{\mathbb{R}^{\infty}}\|\phi\|_{\left(X^{1}\right)^{\prime}}\left\|v_{\mathrm{ev}}(\boldsymbol{y})-\mathcal{I}_{G_{\mathrm{ev}}\left(\xi_{n}\right)} v_{\mathrm{ev}}(\boldsymbol{y})\right\|_{X^{1}} \mathrm{~d} \gamma(\boldsymbol{y}) \\
& \leq C\left\|v_{\mathrm{ev}}-\mathcal{I}_{G_{\mathrm{ev}}\left(\xi_{n}\right)} v_{\mathrm{ev}}\right\|_{\mathcal{L}_{1}\left(X^{1}\right)} \leq C n^{-\min (\alpha, \beta)} .
\end{aligned}
$$

Similarly to the proof of Theorem 4.1, applying (3.42) in Corollary 3.12 for $p=1$, we can derive the following

Corollary 4.2. Under the hypothesis of Corollary 3.11, assume additionally that the sequences $Y_{m}, m \in \mathbb{N}_{0}$, are symmetric. For $\xi>0$, let $\Lambda_{\mathrm{ev}}(\xi)$ be the set defined as in (3.41). Then we have the following.

(i) For each $n \in \mathbb{N}$ there exists a number $\xi_{n}$ such that $\left|\Gamma\left(\Lambda_{\mathrm{ev}}\left(\xi_{n}\right)\right)\right| \leq n$ and

$$
\left\|\int_{\mathbb{R}^{\infty}} v(\boldsymbol{y}) \mathrm{d} \gamma(\boldsymbol{y})-Q_{\Lambda_{\mathrm{ev}}\left(\xi_{n}\right)} v\right\|_{X} \leq C n^{-(1 / q-1 / 2)} .
$$

(ii) Let $\phi \in X^{\prime}$ be a bounded linear functional on $X$. Then for each $n \in \mathbb{N}$ there exists a number $\xi_{n}$ such that $\left|\Gamma\left(\Lambda_{\mathrm{ev}}\left(\xi_{n}\right)\right)\right| \leq n$ and

$$
\left|\int_{\mathbb{R}^{\infty}}\langle\phi, v(\boldsymbol{y})\rangle \mathrm{d} \gamma(\boldsymbol{y})-Q_{\Lambda_{\mathrm{ev}}\left(\xi_{n}\right)}\langle\phi, v\rangle\right| \leq C n^{-(1 / q-1 / 2)} .
$$

The constants $C$ in (4.7) and (4.8) are independent of $v$ and $n$.

\section{Elliptic PDEs with LOGNORMAL inPUts}

In this section, we apply the results in Sections 2-4 to Hermite gpc expansion and polynomial interpolation approximations as well as integration for the parametrized diffusion elliptic equation (1.2) with lognormal inputs (1.3).

We approximate the solution $u(\boldsymbol{y})$ to this equation by truncation of the Hermite series

$$
u(\boldsymbol{y})=\sum_{\boldsymbol{s} \in \mathbb{F}} u_{\boldsymbol{s}} H_{\boldsymbol{s}}(\boldsymbol{y}), \quad u_{\boldsymbol{s}} \in V .
$$

For convenience, we introduce the conventions: $W^{1}:=V ; W^{2}:=W ; H^{0}(D):=L_{2}(D) ; W^{0, \infty}(D):=L_{\infty}(D)$. Constructions of fully discrete approximations and integration are based on the approximation property (1.5) in Assumption 1.1 and the weighted $\ell_{2}$-summability of the series $\left(\left\|u_{\boldsymbol{s}}\right\|_{W^{r}}\right)_{\boldsymbol{s} \in \mathbb{F}}, r=1,2$ in the following lemma which has been proven in [3] for $r=1$ and in [1] for $r=2$.

Lemma 5.1. Let $r=1,2$. Assume that the right side $f$ in (1.2) belongs to $H^{r-2}(D)$, that the domain $D$ has $C^{r-2,1}$ smoothness, that all functions $\psi_{j}$ belong to $W^{r-1, \infty}(D)$. Assume that there exist a number $0<q_{r}<\infty$ and a sequence $\boldsymbol{\rho}_{r}=\left(\rho_{r ; j}\right)_{j \in \mathbb{N}}$ of positive numbers such that $\left(\rho_{r ; j}^{-1}\right)_{j \in \mathbb{N}} \in \ell_{q_{r}}(\mathbb{N})$ and

$$
\sup _{|\alpha| \leq r-1}\left\|\sum_{j \in \mathbb{N}} \rho_{r ; j}\left|D^{\alpha} \psi_{j}\right|\right\|_{L^{\infty}(D)}<\infty .
$$


Then we have that for any $\eta \in \mathbb{N}$,

$$
\sum_{\boldsymbol{s} \in \mathbb{F}}\left(\sigma_{r ; \boldsymbol{s}}\left\|u_{\boldsymbol{s}}\right\|_{W^{r}}\right)^{2}<\infty \quad \text { with } \quad \sigma_{r ; \boldsymbol{s}}^{2}:=\sum_{\left\|\boldsymbol{s}^{\prime}\right\|_{\ell_{\infty}(\mathbb{F})} \leq \eta}\left(\begin{array}{c}
\boldsymbol{s} \\
\boldsymbol{s}^{\prime}
\end{array}\right) \boldsymbol{\rho}_{r}^{2 \boldsymbol{s}^{\prime}} .
$$

We need two auxiliary lemmata.

Lemma 5.2. Let the assumptions of Lemma 5.1 hold for the space $W^{1}$ with $0<q_{1}<2$. Then the solution map $\boldsymbol{y} \mapsto u(\boldsymbol{y})$ is $\gamma$-measurable and $u \in \mathcal{L}_{2}\left(W^{1}\right)$. Moreover, $u \in \mathcal{L}_{2}^{\mathcal{E}}\left(W^{1}\right)$ where

$$
\mathcal{E}:=\left\{\boldsymbol{y} \in \mathbb{R}^{\infty}: \sup _{j \in \mathbb{N}} \rho_{1 ; j}^{-1}\left|y_{j}\right|<\infty\right\}
$$

having $\gamma(\mathcal{E})=1$ and containing all $\boldsymbol{y} \in \mathbb{R}^{\infty}$ with $|\boldsymbol{y}|_{0}<\infty$.

Proof. The proof of this lemma already is in [3]. Indeed, under the assumptions of Lemma 5.1 for the space $W^{1}$ with $0<q_{1}<2$, by Remark 2.5 of [3] Assumption A in Page 349 of [3] holds for the sequence $\boldsymbol{\rho}_{1}=\left(\rho_{1 ; j}\right)_{j \in \mathbb{N}}$. Hence, by Corollary 2.3 of [3] the solution map $\boldsymbol{y} \mapsto u(\boldsymbol{y})$ is $\gamma$-measurable and $u \in \mathcal{L}_{2}\left(W^{1}\right)$. Moreover, $\gamma(\mathcal{E})=1$ (2.23) of [3] and, obviously, $\mathcal{E}$ contains all $\boldsymbol{y} \in \mathbb{R}^{\infty}$ with $|\boldsymbol{y}|_{0}<\infty$. For a point $\boldsymbol{y} \in \mathbb{R}^{\infty}$, by the Lax-Milgram lemma the solution $u(\boldsymbol{y})$ is well-defined if $b(\boldsymbol{y}) \in L_{\infty}(D)$. This inclusion holds true if $\boldsymbol{y} \in \mathcal{E}$ (2.26) of [3]. This means that $u \in \mathcal{L}_{2}^{\mathcal{E}}\left(W^{1}\right)$.

We make use the following notation: for $\nu \in \mathbb{N}$,

$$
\mathbb{F}_{\nu}:=\left\{s \in \mathbb{F}: s_{j} \in \mathbb{N}_{0, \nu}, j \in \mathbb{N}\right\} ; \quad \mathbb{N}_{0, \nu}:=\left\{n \in \mathbb{N}_{0}: n=0, \nu, \nu+1, \ldots\right\} .
$$

The set $\mathbb{F}_{\nu}$ has been introduced in [33]. The set $\mathbb{F}_{2}$ plays an important role in establishing improved convergence rates for sparse-grid Smolyak quadrature in [33,34].

The following lemma is a generalization of Lemma 5.1 in [3].

Lemma 5.3. Let $0<q<\infty, \eta \in \mathbb{N}, \boldsymbol{\rho}=\left(\rho_{j}\right)_{j \in \mathbb{N}}$ of positive numbers such the sequence $\left(\rho_{j}^{-1}\right)_{j \in \mathbb{N}}$ belongs to $\ell_{q}(\mathbb{N})$. Let $\theta, \lambda$ be arbitrary positive numbers and $\left(p_{\boldsymbol{s}}(\theta, \lambda)\right)_{\boldsymbol{s} \in \mathbb{F}}$ the sequence given in (3.3). Let for numbers $\eta \in \mathbb{N}$ the sequence $\left(\sigma_{\boldsymbol{s}}\right)_{\boldsymbol{s} \in \mathbb{F}}$ be defined by

$$
\sigma_{s}^{2}:=\sum_{\left\|s^{\prime}\right\|_{\ell_{\infty}(\mathbb{F})} \leq \eta}\left(\begin{array}{c}
s \\
s^{\prime}
\end{array}\right) \rho^{2 s^{\prime}}
$$

Then for any $\eta>\frac{2 \nu(\theta+1)}{q}$, we have

$$
\sum_{\boldsymbol{s} \in \mathbb{F}_{\nu}} p_{\boldsymbol{s}}(\theta, \lambda) \sigma_{\boldsymbol{s}}^{-q / \nu}<\infty
$$

Proof. With $\theta^{\prime}:=2 \theta \nu / q$, we have that

$$
\begin{aligned}
\sum_{\boldsymbol{s} \in \mathbb{F}_{\nu}} p_{\boldsymbol{s}}(\theta, \lambda) \sigma_{\boldsymbol{s}}^{-q / \nu} & =\sum_{\boldsymbol{s} \in \mathbb{F}_{\nu}} \prod_{j \in \mathbb{N}}\left(\sum_{k=0}^{\eta}\left(\begin{array}{c}
s_{j} \\
k
\end{array}\right)\left(1+\lambda s_{j}\right)^{\theta^{\prime}} \rho_{j}^{2 k}\right)^{-q / 2 \nu} \\
& =\prod_{j \in \mathbb{N}} \sum_{n \in \mathbb{N}_{0, \nu}}\left(\sum_{k=0}^{\eta}\left(\begin{array}{l}
n \\
k
\end{array}\right)(1+\lambda n)^{\theta^{\prime}} \rho_{j}^{2 k}\right)^{-q / 2 \nu}=: \prod_{j \in \mathbb{N}} B_{j}
\end{aligned}
$$


and

$$
\begin{aligned}
B_{j} & \leq \sum_{n \in \mathbb{N}_{0, \nu}}\left(\left(\begin{array}{c}
n \\
\min (n, \eta)
\end{array}\right)(1+\lambda n)^{\theta^{\prime}} \rho_{j}^{2 \min (n, \eta)}\right)^{-q / 2 \nu} \\
& \leq \sum_{n \in \mathbb{N}_{0, \nu}, n<\eta}\left(\left(\begin{array}{c}
n \\
n
\end{array}\right)(1+\lambda n)^{\theta^{\prime}} \rho_{j}^{2 n}\right)^{-q / 2 \nu}+\sum_{n \geq \eta}\left((1+\lambda n)^{\theta^{\prime} \rho_{j}^{2 \eta}}\right)^{-q / 2 \nu} \\
& \leq \sum_{n \in \mathbb{N}_{0, \nu}, n<\eta}(1+\lambda n)^{\theta} \rho_{j}^{-n q / \nu}+\rho_{j}^{-\eta q / \nu} \sum_{n \geq \eta}\left(\begin{array}{l}
n \\
\eta
\end{array}\right)^{-q / 2 \nu}(1+\lambda n)^{\theta}=: B_{j, 1}+B_{j, 2} .
\end{aligned}
$$

We estimate $B_{j, 1}$ and $B_{j, 2}$. We have

$$
B_{j, 1} \leq 1+\sum_{n=\nu}^{\eta-1}(1+\lambda n)^{\theta} \rho_{j}^{-n q / \nu} \leq 1+(1+(\eta-1) \lambda)^{\theta} \sum_{n=\nu}^{\eta-1} \rho_{j}^{-n q / \nu} .
$$

From the inequalities $\left(\frac{n}{\eta}\right)^{\eta} \leq\left(\begin{array}{l}n \\ \eta\end{array}\right)$ and $\eta q / 2 \nu-\theta>1$ we derive that

$$
B_{j, 2} \leq \rho_{j}^{-\eta q / \nu} \sum_{n \geq \eta}\left(\frac{n}{\eta}\right)^{-\eta q / 2 \nu}(1+\lambda n)^{\theta} \leq C \rho_{j}^{-\eta q / \nu} \sum_{n \geq \eta} n^{-(\eta q / 2 \nu-\theta)} \leq C \rho_{j}^{-\eta q / \nu} .
$$

Summing up we obtain that

$$
B_{j} \leq B_{j, 1}+B_{j, 2} \leq 1+C \sum_{n=\nu}^{\eta} \rho_{j}^{-n q / \nu} .
$$

Since the sequence $\left(\rho_{j}^{-1}\right)_{j \in \mathbb{N}}$ belongs to $\ell_{q}(\mathbb{N})$, there exists $j^{*}$ large enough such that $\rho_{j}>1$ for all $j \geq j^{*}$. Hence, there exists a constant $C$ independent of $j$ such that $B_{j} \leq 1+C \rho_{j}^{-q}$ for all $j \in \mathbb{N}$, and consequently,

$$
\sum_{\boldsymbol{s} \in \mathbb{F}_{\nu}} p_{\boldsymbol{s}}(\theta, \lambda) \sigma_{\boldsymbol{s}}^{-q / 2 \nu} \leq \prod_{j \in \mathbb{N}} B_{j} \leq \prod_{j \in \mathbb{N}}\left(1+C \rho_{j}^{-q}\right) \leq \exp \left(\sum_{j \in \mathbb{N}} C \rho_{j}^{-q}\right)<\infty .
$$

In the present paper, as noticed in Introduction we want to show possibilities of non-adaptive approximation methods and convergence rates of approximation by such methods for the parametrized diffusion elliptic equation (1.2) with lognormal inputs. Here we do not consider Galerkin approximations. To treat fully discrete approximations we assume that $f \in L_{2}(D)$ and that it holds the approximation property (1.5) in Assumption 1.1 for all $v \in W$, see, for instance, Theorem 3.2.1 of [8] for the case when $D$ is a polygonal set. Notice that classical error estimates yield the convergence rate $\alpha=1 / d$ by using Lagrange finite elements of order at least 1 on quasi-uniform partitions. Also, the spaces $W$ do not always coincide with $H^{2}(D)$. For example, for $d=2$, we know that $W$ is strictly larger than $H^{2}(D)$ when $D$ is a polygon with re-entrant corner. In this case, it is well known that the optimal rate $\alpha=1 / 2$ is yet attained, when using spaces $V_{n}$ associated to meshes $\left(\mathcal{I}_{n}\right)_{n>0}$ with proper refinement near the re-entrant corners where the functions $v \in W$ might have singularities.

Theorem 5.4. Let $0<p \leq 2$. Let Assumption 1.1 hold. Let the assumptions of Lemma 5.1 hold for the spaces $W^{1}=V$ and $W^{2}=W$ with some $0<q_{1} \leq q_{2}<\infty$. For $\xi>0$, let $G(\xi)$ be the set defined by (2.7) for $\sigma_{r ; s}$ as in (5.1), $r=1,2$.

Then for each $n \in \mathbb{N}$ there exists a number $\xi_{n}$ such that $\operatorname{dim}\left(\mathcal{V}\left(G\left(\xi_{n}\right)\right) \leq n\right.$ and

$$
\left\|u-\mathcal{S}_{G\left(\xi_{n}\right)} u\right\|_{\mathcal{L}_{p}(V)} \leq C n^{-\min (\alpha, \beta)} .
$$

The rate $\alpha$ corresponds to the spatial approximation of a single function in $W$ as given by (1.5), and the rate $\beta$ is given by (2.9). The constant $C$ in (5.2) is independent of $u$ and $n$. 
Proof. To prove the theorem it is sufficient to notice that the assumptions of Theorem 2.3 are satisfied for $X^{1}=V$ and $X^{2}=W$. This can be done by using Lemmata 5.1-5.3. (By multiplying the sequences $\boldsymbol{\rho}_{r}$ in Lemma 5.1 with a positive constant we can get $\sigma_{r ; s}>1$ for $s \in \mathbb{F}$.)

Remark 5.5. (i) The rate $\min (\alpha, \beta)$ in $(5.2)$ is the rate of best adaptive $n$-term Hermite gpc expansion approximation in $\mathcal{L}_{2}(V)$ based on $\ell_{p_{1}}$-summability of $\left(\left\|u_{\boldsymbol{s}}\right\|_{V}\right)_{\boldsymbol{s} \in \mathbb{F}}$ and $\ell_{p_{2}}$-summability of $\left(\left\|u_{\boldsymbol{s}}\right\|_{W}\right)_{\boldsymbol{s} \in \mathbb{F}}$ proven in [1], where $1 / p_{r}=1 / q_{r}+1 / 2$ for $r=1,2$.

(ii) Observe that $\mathcal{S}_{G\left(\xi_{n}\right)}$ can be represented in the form of a multilevel approximation method with $k_{n}$ levels:

$$
\mathcal{S}_{G\left(\xi_{n}\right)}=\sum_{k=0}^{k_{n}} \delta_{k} S_{\Lambda_{k}\left(\xi_{n}\right)}
$$

where $S_{\Lambda} u:=\sum_{s \in \Lambda} u_{\boldsymbol{s}}$ for $\Lambda \subset \mathbb{F}, k_{n}:=\left\lfloor\log _{2} \xi_{n}\right\rfloor$ and for $k \in \mathbb{N}_{0}$ and $\xi>1$,

$$
\Lambda_{k}(\xi):=\left\{\begin{array}{lll}
\left\{s \in \mathbb{F}: \sigma_{2 ; s}^{q_{2}} \leq 2^{-k} \xi\right\} & \text { if } \quad \alpha \leq 1 / q_{2} \\
\left\{s \in \mathbb{F}: \sigma_{1 ; s}^{q_{1}} \leq \xi, \sigma_{2 ; s}^{q_{1}} \leq 2^{-\alpha q_{1} k} \xi\right\} & \text { if } \quad \alpha>1 / q_{2} .
\end{array}\right.
$$

Remark 5.6. Since the index set $G(\xi)$ defined as in (2.7) plays a key role in determining the operator $\mathcal{S}_{G(\xi)}$, we give an algorithm for constructing it, for instance, for the case $\alpha>1 / q_{2}$. The case $\alpha \leq 1 / q_{2}$ can be done similarly. We additionally assume that the sequences $\rho_{r ; s}, r=1,2$, are monotonically increasing. This assumption yields that if $\boldsymbol{s} \in \mathbb{F}$ and $i<j$ are such that $s_{i}=s_{j}=0$, than $\sigma_{r ; \boldsymbol{s}+\boldsymbol{e}_{i}} \leq \sigma_{r ; \boldsymbol{s}+\boldsymbol{e}_{j}}, r=1,2$, where $\boldsymbol{e}_{j}:=\left(\delta_{i, j}\right)_{i \in \mathbb{N}} \in \mathbb{F}$. Observe that

$$
G(\xi)=\bigcup_{k=0}^{k_{\xi}}\left\{(k, s) \in \mathbb{N}_{0} \times \mathbb{F}: s \in \Lambda_{k}(\xi)\right\},
$$

where $k_{\xi}:=\left\lfloor\frac{1}{\alpha q_{1}} \log _{2} \xi\right\rfloor$ and $\Lambda_{k}(\xi)$ is defined as in (5.3), $\xi>1$. Moreover, $\Lambda_{k}(\xi)$ are downward closed sets, and consequently, the sequence $\left\{\Lambda_{k}(\xi)\right\}_{k=0}^{k_{\xi}}$ is nested in the inverse order, i.e., $\Lambda_{k^{\prime}}(\xi) \subset \Lambda_{k}(\xi)$ if $k^{\prime}>k$, and $\Lambda_{0}(\xi)$ is the largest and $\Lambda_{k_{\xi}}(\xi)$ is the smallest. Hence, the index set $G(\xi)$ can be constructed as in Algorithm 1.

Let us estimate the computational complexity of Algorithm 1, by using some results from Lemmata 3.1.12 and 3.1.13 of [32]. Each from 1st to 5th lines and 10th to 21st lines in this algorithm is executed at most $4\left|\Lambda_{0}(\xi)\right|+1$ times. For every multiindex $s \in \Lambda_{0}(\xi)$ we store $\left\{\left(j, s_{j}\right): s_{j} \neq 0\right\}$. Each multiindex therefore occupies a memory of size $\mathcal{O}\left(d\left(\Lambda_{0}(\xi)\right)\right)$, where $d(\Lambda):=\sup _{\boldsymbol{s} \in \Lambda}|\operatorname{supp} \boldsymbol{s}|$. Assuming elementary operations such as multiplications and divisions to be of complexity $\mathcal{O}(1)$, we can deduce that the computational complexity executing each from 1st to 5th lines and 10th to 21st lines in Algorithm 1 is bounded by $\mathcal{O}\left(m\left(\Lambda_{0}(\xi)\right)+d\left(\Lambda_{0}(\xi)\right)\right)$, where $m(\Lambda):=\sup _{s \in \Lambda}\left|\sum_{j \in \mathbb{N}} s_{j}\right|$. Algorithm 1 terminates and gives $G(\xi)$ before $k>k_{\xi}$. Hence the overall computational complexity and memory consumption of Algorithm 1 behave like

$$
\mathcal{O}\left(k_{\xi}\left|\Lambda_{0}(\xi)\right|\left(m\left(\Lambda_{0}(\xi)\right)+d\left(\Lambda_{0}(\xi)\right)\right)\right)=\mathcal{O}\left(\log _{2} \xi \sum_{\boldsymbol{s} \in \Lambda_{0}(\xi)} p_{\boldsymbol{s}}(1,1)\right)=\mathcal{O}\left(\xi \log _{2} \xi\right)
$$

In the last step we used the equality $\sum_{\sigma_{1, s} \leq \xi^{1 / q_{1}}}=\mathcal{O}(\xi)$ which follows from the inequality $\sum_{\boldsymbol{s} \in \mathbb{F}} p_{\boldsymbol{s}}(1,1) \sigma_{1, \boldsymbol{s}}^{-q_{1}}<$ $\infty(c f .(3.40))$.

We now consider the problem of sparse-grid interpolation approximation and intergration of the solution $u(\boldsymbol{y})$ to the parametrized diffusion elliptic equation (1.2) with lognormal inputs. By using Lemmata 5.1-5.3, in the same way as the proof of Theorem 5.4, from Theorems 2.3 and 3.8 and Corollary 3.11 we derive the following two theorems and two corollaries. 


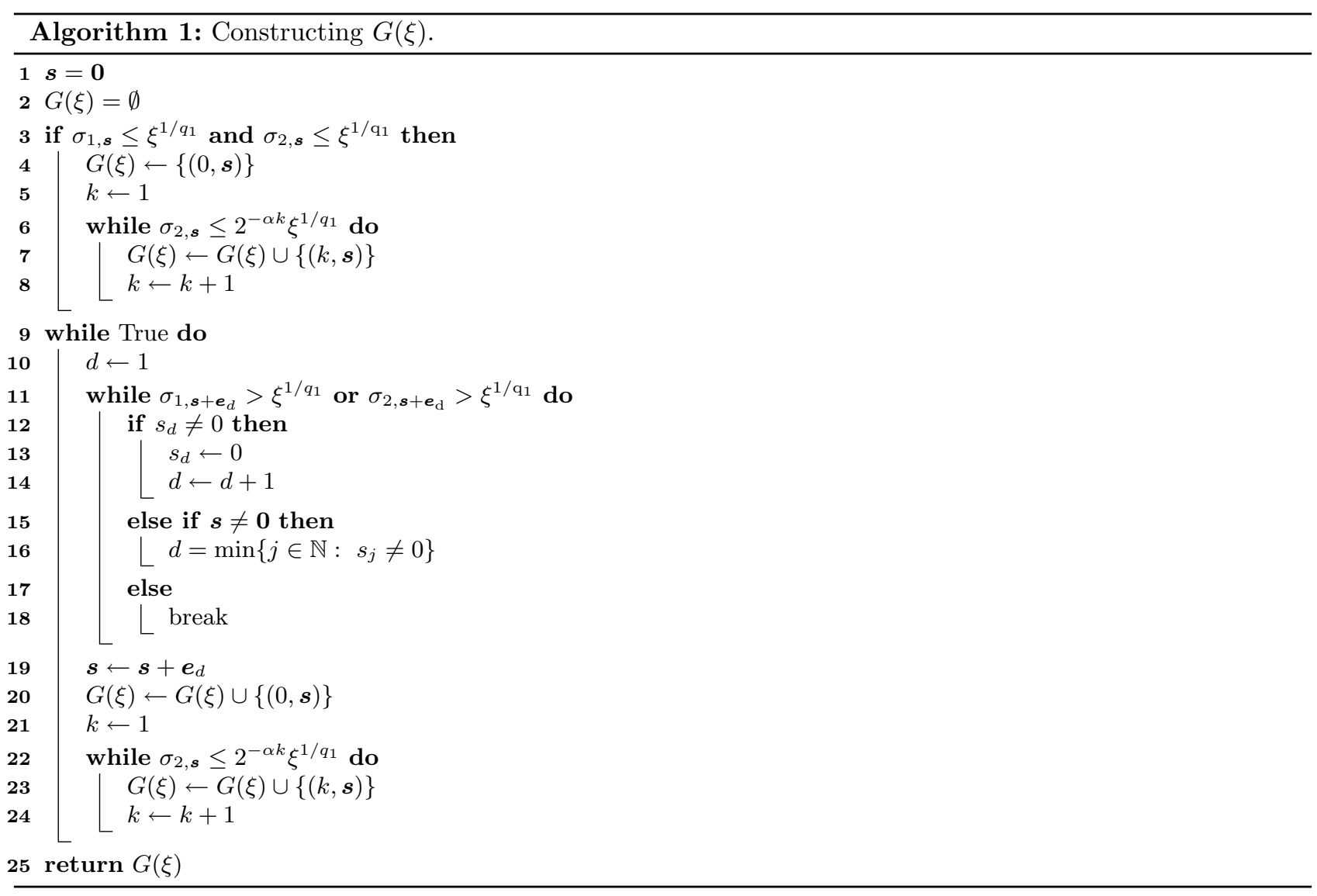

Theorem 5.7. Let $0<p \leq 2$. Let Assumption 1.1 hold. Let the assumptions of Lemma 5.1 hold for the spaces $W^{1}=V$ and $W^{2}=W$ with some $0<q_{1} \leq q_{2}<\infty$ with $q_{1}<2$. Assume that $\left(Y_{m}\right)_{m \in \mathbb{N}_{0}}$ is a sequence satisfying the condition (3.15) for some positive numbers $\tau$ and $C$. For $\xi>0$, let $G(\xi)$ be the set defined by (3.5) for $\sigma_{r ; s}$ as in (5.1), $r=1,2$.

Then for each $n \in \mathbb{N}$ there exists a number $\xi_{n}$ such that for the interpolation operator $\mathcal{I}_{G\left(\xi_{n}\right)}: \mathcal{L}_{2}^{\mathcal{E}}(W) \rightarrow$ $\mathcal{V}\left(G\left(\xi_{n}\right)\right)$, we have that $\operatorname{dim} \mathcal{V}\left(G\left(\xi_{n}\right)\right) \leq n$ and

$$
\left\|u-\mathcal{I}_{G\left(\xi_{n}\right)} u\right\|_{\mathcal{L}_{p}(V)} \leq C n^{-\min (\alpha, \beta)} .
$$

The rate $\alpha$ corresponds to the spatial approximation of a single function in $W$ as given by (1.5). The rate $\beta$ is given by (3.20). The constant $C$ in (5.4) is independent of $u$ and $n$.

Remark 5.8. (i) Observe that $\mathcal{I}_{G\left(\xi_{n}\right)}$ can be represented in the form of a multilevel approximation method, see Remark 3.9(i) for details.

(ii) The fully discrete polynomial interpolation approximation by operators $\mathcal{I}_{G\left(\xi_{n}\right)}$ is a collocation approximation based on the finite number $\left|\Gamma\left(\Lambda_{0}\left(\xi_{n}\right)\right)\right| \leq \sum_{\boldsymbol{s} \in \Lambda_{0}\left(\xi_{n}\right)} p_{\boldsymbol{s}}(1,2)$ of the particular solvers $u(\boldsymbol{y}), \boldsymbol{y} \in \Gamma\left(\Lambda_{0}\left(\xi_{n}\right)\right)$, where, we recall, $\Gamma\left(\Lambda_{0}\left(\xi_{n}\right)\right)=\cup_{\boldsymbol{s} \in \Lambda_{0}\left(\xi_{n}\right)} \Gamma_{\boldsymbol{s}}$ and $\Gamma_{\boldsymbol{s}}=\left\{\boldsymbol{y}_{\boldsymbol{s}-\boldsymbol{e} ; \boldsymbol{m}}: e \in \mathbb{E}_{\boldsymbol{s}} ; m_{j}=0, \ldots, s_{j}-e_{j}, j \in \mathbb{N}\right\}$. $\left(\mathbb{E}_{\boldsymbol{s}}\right.$ denotes the subset in $\mathbb{F}$ of all $\boldsymbol{e}$ such that $e_{j}$ is 1 or 0 if $s_{j}>0$, and $e_{j}$ is 0 if $s_{j}=0$, and $\boldsymbol{y}_{\boldsymbol{s} ; \boldsymbol{m}}:=\left(y_{s_{j} ; m_{j}}\right)_{j \in \mathbb{N}}$.)

Corollary 5.9. Let $0<p \leq 2$. Under the hypothesis of Lemma 5.1 for the spaces $W^{1}=V$ with some $0<q_{1}=$ $q<2$. For $\xi>0$, let $\Lambda(\xi)$ be the set defined by (3.38) for $\sigma_{s}=\sigma_{1 ; s}$ as in (5.1). Then for each $n \in \mathbb{N}$ there 
exists a number $\xi_{n}$ such that $\left|\Gamma\left(\Lambda\left(\xi_{n}\right)\right)\right| \leq n$ and

$$
\left\|u-I_{\Lambda\left(\xi_{n}\right)} u\right\|_{\mathcal{L}_{p}(V)} \leq C n^{-(1 / q-1 / 2)} .
$$

The constant $C$ in (5.5) is independent of $u$ and $n$.

The rate $1 / q-1 / 2$ in Corollary 5.9 is much better than the rate $\frac{1}{2}(1 / q-1 / 2)$ which has been obtained in Theorem 3.18 of [18] for a similar approximation in $\mathcal{L}_{2}(V)$.

Similarly to $\mathcal{I}_{G\left(\xi_{n}\right)}$, the approximation to $u$ by the operator $I_{\Lambda\left(\xi_{n}\right)}$, is a collocation approximation based on the finite number $\left|\Gamma\left(\Lambda\left(\xi_{n}\right)\right)\right| \leq \sum_{\boldsymbol{s} \in \Lambda\left(\xi_{n}\right)} p_{\boldsymbol{s}}(1,2)$ of the particular solvers $u(\boldsymbol{y}), \boldsymbol{y} \in \Gamma\left(\Lambda\left(\xi_{n}\right)\right)$.

Theorem 5.10. Let Assumption 1.1 hold. Let the assumptions of Lemma 5.1 hold for the spaces $W^{1}=V$ and $W^{2}=W$ with some $0<q_{1} \leq q_{2}<\infty$ with $q_{1}<4$. Assume that $\left(Y_{m}\right)_{m \in \mathbb{N}_{0}}$ is a sequence satisfying the condition (3.15) for some positive numbers $\tau$ and $C$, and such that $Y_{m}$ is summetric for every $m \in \mathbb{N}_{0}$. For $\xi>0$, let $G_{\mathrm{ev}}(\xi)$ be the set defined by (3.11) for $\sigma_{r ; s}$ as in (5.1), $r=1,2$. Then for the quadrature operator $\mathcal{Q}_{G_{\mathrm{ev}}(\xi)}$ generated by the interpolation operator $\mathcal{I}_{G_{\mathrm{ev}}(\xi)}: \mathcal{L}_{2}^{\mathcal{E}}(W) \rightarrow \mathcal{V}\left(G_{\mathrm{ev}}(\xi)\right)$, we have the following.

(i) For each $n \in \mathbb{N}$ there exists a number $\xi_{n}$ such that $\operatorname{dim} \mathcal{V}\left(G_{\mathrm{ev}}\left(\xi_{n}\right)\right) \leq n$ and

$$
\left\|\int_{\mathbb{R}^{\infty}} v(\boldsymbol{y}) \mathrm{d} \gamma(\boldsymbol{y})-\mathcal{Q}_{G_{\mathrm{ev}}\left(\xi_{n}\right)} v\right\|_{V} \leq C n^{-\min (\alpha, \beta)} .
$$

(ii) Let $\phi \in V^{\prime}$ be a bounded linear functional on $V$. Then for each $n \in \mathbb{N}$ there exists a number $\xi_{n}$ such that $\operatorname{dim} \mathcal{V}\left(G_{\mathrm{ev}}\left(\xi_{n}\right)\right) \leq n$ and

$$
\left|\int_{\mathbb{R}^{\infty}}\langle\phi, v(\boldsymbol{y})\rangle \mathrm{d} \gamma(\boldsymbol{y})-\mathcal{Q}_{G_{\mathrm{ev}}\left(\xi_{n}\right)}\langle\phi, v\rangle\right| \leq C n^{-\min (\alpha, \beta)} .
$$

The rate $\alpha$ corresponds to the spatial approximation of a single function in $W$ as given by (1.5). The rate $\beta$ is given by

$$
\beta:=\left(\frac{2}{q_{1}}-\frac{1}{2}\right) \frac{\alpha}{\alpha+\delta}, \quad \delta:=\frac{2}{q_{1}}-\frac{2}{q_{2}} .
$$

The constants $C$ in (5.6) and (5.7) are independent of $u$ and $n$.

Proof. Observe that $\mathbb{F}_{\mathrm{ev}} \subset \mathbb{F}_{2}$. From Lemmata 5.1 and 5.3 we can see that the assumptions of Theorem 3.8 hold for $X^{1}=V$ and $X^{2}=W$ with $0<q_{1} / 2 \leq q_{2} / 2<\infty$ and $q_{1} / 2<2$. Hence, by applying Theorem 4.1 we prove the theorem.

Observe that the rate in (5.6) and (5.7) can be improved as $\min \left(\alpha, \frac{2}{q_{1}} \frac{\alpha}{\alpha+\delta}\right)$ if the sequences $\left(\left\|u_{\boldsymbol{s}}\right\|_{V}\right)_{\boldsymbol{s} \in \mathbb{F}}$ and $\left(\left\|u_{\boldsymbol{s}}\right\|_{W^{r}}\right)_{\boldsymbol{s} \in \mathbb{F}}$ have $\ell_{p_{1}}$ - and $\ell_{p_{r}}$-summable majorant sequences, respectively, where $1 / p_{1}=1 / q_{1}+1 / 2$ and $1 / p_{r}=1 / q_{r}+1 / 2$. Similarly to $\mathcal{I}_{G\left(\xi_{n}\right)}$, the quadrature operator $\mathcal{Q}_{G_{\mathrm{ev}}\left(\xi_{n}\right)}$ can be represented in the form of a multilevel integration method with $k_{n}$ levels:

$$
\mathcal{Q}_{G_{\mathrm{ev}}\left(\xi_{n}\right)}=\sum_{k=0}^{k_{n}} \delta_{k} Q_{\Lambda_{\mathrm{ev} ; k}\left(\xi_{n}\right)},
$$

where $k_{n}:=\left\lfloor\log _{2} \xi_{n}\right\rfloor$ and for $k \in \mathbb{N}_{0}$ and $\xi>0$,

$$
\Lambda_{\mathrm{ev} ; k}(\xi):= \begin{cases}\left\{s \in \mathbb{F}_{\mathrm{ev}}: \sigma_{2 ; s}^{q_{2}} \leq 2^{-k} \xi\right\} & \text { if } \quad \alpha \leq 1 / q_{2}-1 / 2 \\ \left\{\boldsymbol{s} \in \mathbb{F}_{\mathrm{ev}}: \sigma_{1 ; s}^{q_{1}} \leq \xi, \sigma_{2 ; s} \leq 2^{-(\alpha+1 / 2) k} \xi^{\vartheta}\right\} & \text { if } \quad \alpha>1 / q_{2}-1 / 2 .\end{cases}
$$

In the same way, from Corollary 4.2 we derive the following 
Corollary 5.11. Let the assumptions of Lemma 5.1 hold for the spaces $W^{1}=V$ with some $0<q_{1}=q<4$. Assume that $\left(Y_{m}\right)_{m \in \mathbb{N}_{0}}$ is a sequence satisfying the condition (3.15) for some positive numbers $\tau$ and $C$, and such that $Y_{m}$ is summetric for every $m \in \mathbb{N}_{0}$. For $\xi>0$, let $\Lambda_{\mathrm{ev}}(\xi)$ be the set defined by (3.41) for $\sigma_{\boldsymbol{s}}=\sigma_{1 ; \boldsymbol{s}}$ as in (5.1). Then we have the following.

(i) For each $n \in \mathbb{N}$ there exists a number $\xi_{n}$ such that $\left|\Gamma\left(\Lambda_{\mathrm{ev}}\left(\xi_{n}\right)\right)\right| \leq n$ and

$$
\left\|\int_{\mathbb{R}^{\infty}} u(\boldsymbol{y}) \mathrm{d} \gamma(\boldsymbol{y})-Q_{\Lambda_{\mathrm{ev}}\left(\xi_{n}\right)} u\right\|_{V} \leq C n^{-(2 / q-1 / 2)}
$$

(ii) Let $\phi \in V^{\prime}$ a bounded linear functional on $V$. For each $n \in \mathbb{N}$ there exists a number $\xi_{n}$ such that $\left|\Gamma\left(\Lambda_{\mathrm{ev}}\left(\xi_{n}\right)\right)\right| \leq n$ and

$$
\left|\int_{\mathbb{R}^{\infty}}\langle\phi, u(\boldsymbol{y})\rangle \mathrm{d} \gamma(\boldsymbol{y})-Q_{\Lambda_{\mathrm{ev}}\left(\xi_{n}\right)}\langle\phi, u\rangle\right| \leq C n^{-(2 / q-1 / 2)}
$$

The constants $C$ in (5.8) and (5.9) are independent of $u$ and $n$.

Remark 5.12. (i) As noticed in Section 4, the sparsity of the grids $\Gamma\left(\Lambda_{\mathrm{ev} ; 0}(\xi)\right)$ and $\Gamma\left(\Lambda_{\mathrm{ev}}(\xi)\right)$ of the evaluation points in the quadrature operators $\mathcal{Q}_{G_{\mathrm{ev}}(\xi)}$ and $Q_{\Lambda_{\mathrm{ev}}(\xi)}$ are much higher than the sparsity of the grids $\Gamma\left(\Lambda_{0}(\xi)\right)$ and $\Gamma(\Lambda(\xi))$ of the evaluation points in the generating interpolation operators $\mathcal{I}_{G(\xi)}$ and $I_{\Lambda(\xi)}$.

(ii) The rate $2 / q-1 / 2$ in Corollary 5.11 is a significant improvement of the rate $\frac{1}{2}(1 / q-1 / 2)$ which has been recently obtained in Corollary 3.12 of [4].

(iii) Since the use and analysis of non-adaptive construction methods for sparse-grid interpolation are important, let us compare in details our methods in Corollary 5.11 with those which has been also discussed in [4]. To construct a quadrature of the form $Q_{\Lambda}$, the author of the last work used the set $\Lambda_{m} \subset \mathbb{F}$ of all indices $s$ (including non-even) corresponding to the $m$ smallest values of $\sigma_{s}$. The number $n=n(m)$ of quadrature points in $\Lambda_{m} \subset \mathbb{F}$ is estimated as $n \leq C m^{2}$ Proposition 3.16 of [18]. This lead to the rate $\frac{1}{2}(1 / q-1 / 2)$. In the present paper, we used the set $\Lambda_{\text {ev }}(\xi) \subset \mathbb{F}_{\text {ev }}$ of all only even indices $s$ by thresholding $\sigma_{s} \leq \xi^{1 / q}$. Formally, this is similar to choosing all even indices $s$ corresponding to the smallest values of $\sigma_{s}$ satisfying $\sigma_{s} \leq \xi^{1 / q}$. Then for a given $n \in \mathbb{N}$, we selected a number $\xi_{n}$ such that the number of quadrature points in the grid $\Gamma\left(\Lambda_{\text {ev }}\left(\xi_{n}\right)\right)$ does not exceed $n$. Hence, due to the evenness of the indices in the set $\Lambda_{\text {ev }}\left(\xi_{n}\right)$ we obtained the improved rate $2 / q-1 / 2$ and that the sparsity of $\Lambda_{\mathrm{ev}}\left(\xi_{n}\right)$ is much higher then that of $\Lambda_{m(n)}$.

\section{Elliptic PDEs with AFFine inPuts}

The theory of non-adaptive approximation and integration of functions in Bochner spaces with infinite tensor product Gaussian measure in Sections 2-4 can be generalized and extended to other situations. In this section, we present some results on similar problems for the parametrized diffusion elliptic equation (1.2) with the affine inputs (1.4).

In the affine case, for given $a, b>-1$, we consider the orthogonal Jacobi expansion of the solution $u(\boldsymbol{y})$ of the form

$$
\sum_{\boldsymbol{s} \in \mathbb{F}} u_{\boldsymbol{s}} J_{\boldsymbol{s}}(\boldsymbol{y}), \quad J_{\boldsymbol{s}}(\boldsymbol{y})=\bigotimes_{j \in \mathbb{N}} J_{s_{j}}\left(y_{j}\right), \quad u_{\boldsymbol{s}}:=\int_{\mathbb{I}} u(\boldsymbol{y}) J_{\boldsymbol{s}}(\boldsymbol{y}) \mathrm{d} \nu_{a, b}(\boldsymbol{y}),
$$

where

$$
\begin{gathered}
\mathrm{d} \nu_{a, b}(\boldsymbol{y}):=\bigotimes_{j \in \mathbb{N}} \delta_{a, b}\left(y_{j}\right) \mathrm{d} y_{j}, \\
\delta_{a, b}(y):=c_{a, b}(1-y)^{a}(1+y)^{b}, \quad c_{a, b}:=\frac{\Gamma(a+b+2)}{2^{a+b+1} \Gamma(a+1) \Gamma(b+1)},
\end{gathered}
$$


and $\left(J_{k}\right)_{k \geq 0}$ is the sequence of Jacobi polynomials on $\mathbb{I}:=[-1,1]$ normalized with respect to the Jacobi probability measure $\int_{\mathbb{I}}\left|J_{k}(y)\right|^{2} \delta_{a, b}(y) \mathrm{d} y=1$. One has the Rodrigues' formula

$$
J_{k}(y)=\frac{c_{k}^{a, b}}{k ! 2^{k}}(1-y)^{-a}(1+y)^{-b} \frac{\mathrm{d}^{k}}{\mathrm{~d} y^{k}}\left(\left(y^{2}-1\right)^{k}(1-y)^{a}(1+y)^{b}\right),
$$

where $c_{0}^{a, b}:=1$ and

$$
c_{k}^{a, b}:=\sqrt{\frac{(2 k+a+b+1) k ! \Gamma(k+a+b+1) \Gamma(a+1) \Gamma(b+1)}{\Gamma(k+a+1) \Gamma(k+b+1) \Gamma(a+b+2)}}, k \in \mathbb{N} .
$$

Examples corresponding to the values $a=b=0$ is the family of the Legendre polynomials, and to the values $a=b=-1 / 2$ the family of the Chebyshev polynomials.

We introduce the space $W^{r}:=\left\{v \in V: \Delta v \in H^{r-2}(D)\right\}$ for $r \geq 2$ with the convention $W^{1}:=V$. This space is equipped with the norm $\|v\|_{W^{r}}:=\|\Delta v\|_{H^{r-2}(D)}$, and coincides with the Sobolev space $V \cap H^{r-2}(D)$ with equivalent norms if the domain $D$ has $C^{r-1,1}$ smoothness, see Theorem 2.5.1.1 of [19]. The following lemma has been proven in [2] for $r=1$ and in [1] for $r>1$.

Lemma 6.1. For a given $r \in \mathbb{N}$, assume that $\bar{a} \in L^{\infty}(D)$ is such that $\operatorname{ess} \inf \bar{a}>0$, and that there exists a sequence $\boldsymbol{\rho}_{r}=\left(\rho_{r ; j}\right)_{j \in \mathbb{N}}$ of positive numbers such that

$$
\left\|\frac{\sum_{j \in \mathbb{N}} \rho_{1 ; j}\left|\psi_{j}\right|}{\bar{a}}\right\|_{L^{\infty}(D)}<1 .
$$

Assume that the right side $f$ in $(1.2)$ belongs to $H^{r-2}(D)$, that the domain $D$ has $C^{r-2,1}$ smoothness, that $\bar{a}$ and all functions $\psi_{j}$ belong to $W^{r-1, \infty}(D)$ and that

$$
\sup _{|\alpha| \leq r-1}\left\|\sum_{j \in \mathbb{N}} \rho_{r ; j}\left|D^{\alpha} \psi_{j}\right|\right\|_{L^{\infty}(D)}<\infty .
$$

Then

$$
\sum_{\boldsymbol{s} \in \mathbb{F}}\left(\sigma_{r ; \boldsymbol{s}}\left\|u_{\boldsymbol{s}}\right\|_{W^{r}}\right)^{2}<\infty, \quad \beta_{r ; \boldsymbol{s}}:=\boldsymbol{\rho}_{r}^{s} \prod_{j \in \mathbb{N}} c_{s_{j}}^{a, b} .
$$

Lemma 6.2. Let $0<q<\infty, \boldsymbol{\rho}=\left(\rho_{j}\right)_{j \in \mathbb{N}}$ of numbers larger than 1 such the sequence $\left(\rho_{j}^{-1}\right)_{j \in \mathbb{N}}$ belongs to $\ell_{q}(\mathbb{N}),\left(p_{\boldsymbol{s}}(\theta, \lambda)\right)_{\boldsymbol{s} \in \mathbb{F}}$ is a sequence of the form (3.3) with arbitrary nonnegative $\theta, \lambda$. Then for every $\nu \in \mathbb{N}_{0}$, we have

$$
\sum_{\boldsymbol{s} \in \mathbb{F}_{\nu}} p_{\boldsymbol{s}}(\theta, \lambda)\left(\rho^{-\boldsymbol{s}}\right)^{q / \nu}<\infty
$$

Proof. We have

$$
\sum_{\boldsymbol{s} \in \mathbb{F}_{\nu}} p_{\boldsymbol{s}}(\theta, \lambda)\left(\rho^{-\boldsymbol{s}}\right)^{q / \nu}=\prod_{j \in \mathbb{N}} \sum_{s_{j} \in \mathbb{N}_{0, \nu}} \rho_{j}^{-s_{j} q / \nu}\left(1+\lambda s_{j}\right)^{\theta}=: \prod_{j \in \mathbb{N}} A_{j} .
$$

Since $\boldsymbol{\rho}=\left(\rho_{j}\right)_{j \in \mathbb{N}}$ of numbers larger than one, and such the sequens $\left(\rho_{j}^{-1}\right)_{j \in \mathbb{N}}$ belongs to $\ell_{q}(\mathbb{N})$, we have $\min _{j \in \mathbb{N}} \rho_{j}>1$. Hence, there exists a constant $C$ independent of $j$ such that

$$
A_{j}=1+\sum_{k=\nu}^{\infty} \rho_{j}^{-k q / \nu}(1+\lambda k)^{\theta} \leq 1+C \rho_{j}^{-q}
$$

and consequently,

$$
\sum_{\boldsymbol{s} \in \mathbb{F}_{\nu}} p_{\boldsymbol{s}}(\theta, \lambda)\left(\rho^{-\boldsymbol{s}}\right)^{q / \nu} \leq \prod_{j \in \mathbb{N}}\left(1+C \rho_{j}^{-q}\right) \leq \exp \left(\sum_{j \in \mathbb{N}} C \rho_{j}^{-q}\right)<\infty
$$


We assume that there holds the following approximation property for $V$ and $W^{r}$ with $r>1$.

Assumption 6.3. There are a sequence $\left(V_{n}\right)_{n \in \mathbb{N}_{0}}$ of subspaces $V_{n} \subset V$ of dimension $\leq n$, and a sequence $\left(P_{n}\right)_{n \in \mathbb{N}_{0}}$ of linear operators from $V$ into $V_{n}$, and a number $\alpha>0$ such that

$$
\left\|P_{n}(v)\right\|_{V} \leq C\|v\|_{V}, \quad\left\|v-P_{n}(v)\right\|_{V} \leq C n^{-\alpha}\|v\|_{W^{r}}, \quad \forall n \in \mathbb{N}_{0}, \quad \forall v \in W^{r} .
$$

In this section, we make use the abbreviations: $\mathcal{L}_{p}(V):=L_{p}\left(\mathbb{I}^{\infty}, V, \nu_{a, b}\right)$ and $\mathcal{L}_{p}\left(W^{r}\right):=L_{p}\left(\mathbb{I}^{\infty}, W^{r}, \nu_{a, b}\right)$ and assume that $r>1$. From Lemmata 6.1 and 6.2 we can prove the following results on non-adaptive fully and non-fully discrete Jacobi gpc expansion and polynomial interpolation approximations and integration for the affine case.

Theorem 6.4. Let $0<p \leq 2$. Let Assumption 6.3 hold. Let the assumptions of Lemma 6.1 hold for the spaces $W^{1}=V$ and $W^{r}$ with some $0<q_{1} \leq q_{r}<\infty$. For $\xi>0$, let $G(\xi)$ be the set defined by (2.7) for $\sigma_{1 ; s}:=\beta_{1 ; s}$ and $\sigma_{2 ; s}:=\beta_{r ; s}$ as in (6.1). Then for each $n \in \mathbb{N}$ there exists a number $\xi_{n}$ such that $\operatorname{dim}\left(\mathcal{V}\left(G\left(\xi_{n}\right)\right) \leq n\right.$ and

$$
\left\|u-\mathcal{S}_{G\left(\xi_{n}\right)} u\right\|_{\mathcal{L}_{p}(V)} \leq C n^{-\min (\alpha, \beta)} .
$$

The rate $\alpha$ corresponds to the spatial approximation of a single function in $W^{r}$ as given by (6.2), and the rate $\beta$ is given by (2.9). The constant $C$ in (6.3) is independent of $u$ and $n$.

The rate $\min (\alpha, \beta)$ in (6.3) is the same rate of fully discrete best adaptive $n$-term approximation in $\mathcal{L}_{2}(V)$ based on $\ell_{p_{1}}$-summability of $\left(\left\|u_{s}\right\|_{V}\right)_{s \in \mathbb{F}}$ and $\ell_{p_{r}}$-summability of $\left(\left\|u_{s}\right\|_{W^{r}}\right)_{s \in \mathbb{F}}$ proven in [1], where $1 / p_{1}=$ $1 / q_{1}+1 / 2$ and $1 / p_{r}=1 / q_{r}+1 / 2$. This rate can be achieved by linear fully discrete non-adaptive approximation when $\left(\left\|u_{\boldsymbol{s}}\right\|_{V}\right)_{s \in \mathbb{F}}$ and $\left(\left\|u_{\boldsymbol{s}}\right\|_{W^{r}}\right)_{s \in \mathbb{F}}$ have $\ell_{p_{1}}$-summable and $\ell_{p_{r}}$-summable majorant sequences, respectively [34].

Theorem 6.5. Let $1 \leq p \leq \infty$. Let Assumption 6.3 hold. Let the assumptions of Lemma 6.1 hold for the spaces $W^{1}=V$ and $W^{r}$ with some $0<q_{1} \leq q_{r}<\infty$ with $q_{1}<2$. For $\xi>0$, let $G(\xi)$ be the set defined by in (3.5) for $\sigma_{1 ; s}:=\beta_{1 ; \boldsymbol{s}}$ and $\sigma_{2 ; s}:=\beta_{r ; \boldsymbol{s}}$ as in (6.1). Then for each $n \in \mathbb{N}$ there exists a number $\xi_{n}$ such that $\operatorname{dim}\left(\mathcal{V}\left(G\left(\xi_{n}\right)\right) \leq n\right.$ and

$$
\left\|u-\mathcal{S}_{G\left(\xi_{n}\right)} u\right\|_{\mathcal{L}_{p}(V)} \leq C n^{-\min (\alpha, \beta)} .
$$

The rate $\alpha$ corresponds to the spatial approximation of a single function in $W^{r}$ as given by (6.2). The rate $\beta$ is given by (3.20). The constant $C$ in (6.4) is independent of $u$ and $n$.

For polynomial interpolation approximation and integration, we keep all definitions and notations in Section 3 with a proper modification for the affine case. For example, for univariate interpolation and integration we take a sequence of points $Y_{m}=\left(y_{m ; k}\right)_{k=0}^{m}$ in $\mathbb{I}$ such that

$$
-\infty<y_{m ; 0}<\cdots<y_{m ; m-1}<y_{m ; m}<+\infty ; \quad y_{0 ; 0}=0 .
$$

Sequences of points $Y_{m}=\left(y_{m ; k}\right)_{k=0}^{m}$ satisfying the inequality (3.15), are the symmetric sequences of the Chebyshev points, the symmetric sequences of the Gauss-Lobatto (Clenshaw-Curtis) points and the nested sequence of the $\Re$-Leja points, see [9] for details.

Theorem 6.6. Let $1 \leq p \leq \infty$. Let Assumption 6.3 hold. Let the assumptions of Lemma 6.1 hold for the spaces $W^{1}=V$ and $W^{r}$ with some $0<q_{1} \leq q_{r}<\infty$ with $q_{1}<2$. Assume that $\left(Y_{m}\right)_{m \in \mathbb{N}_{0}}$ is a sequence satisfying the condition (3.15) for some positive numbers $\tau$ and $C$. For $\xi>0$, let $G(\xi)$ be the set defined by (3.5) for $\sigma_{1 ; s}:=\beta_{1 ; s}$ and $\sigma_{2 ; s}:=\beta_{r ; s}$ as in (6.1). Then for each $n \in \mathbb{N}$ there exists a number $\xi_{n}$ such that for the interpolation operator $\mathcal{I}_{G\left(\xi_{n}\right)}: \mathcal{L}_{2}\left(W^{r}\right) \rightarrow \mathcal{V}\left(G\left(\xi_{n}\right)\right.$, we have that $\operatorname{dim} \mathcal{V}\left(G\left(\xi_{n}\right)\right) \leq n$ and

$$
\left\|u-\mathcal{I}_{G\left(\xi_{n}\right)} u\right\|_{\mathcal{L}_{p}(V)} \leq C n^{-\min (\alpha, \beta)} .
$$

The rate $\alpha$ corresponds to the spatial approximation of a single function in $W^{r}$ as given by (6.2). The rate $\beta$ is given by (3.20). The constant $C$ in (6.5) is independent of $u$ and $n$. 
The rates in (6.3)-(6.5) for some non-adaptive approximations have been proven in the case when $\left(\left\|u_{\boldsymbol{s}}\right\|_{V}\right)_{\boldsymbol{s} \in \mathbb{F}}$ and $\left(\left\|u_{s}\right\|_{W^{r}}\right)_{s \in \mathbb{F}}$ have $\ell_{p_{1}}$-summable and $\ell_{p_{r}}$-summable majorant sequences, respectively, which are derived from the analyticity of the solution $u$, where $1 / p_{1}=1 / q_{1}+1 / 2$ and $1 / p_{r}=1 / q_{r}+1 / 2$, see [34].

Theorem 6.7. Let Assumption 6.3 hold. Let $a=b$ for the Jacobi probability measure $\nu_{a, b}(\boldsymbol{y})$, and the assumptions of Lemma 6.1 hold for the spaces $W^{1}=V$ and $W^{r}$ with some $0<q_{1} \leq q_{r}<\infty$ with $q_{1}<4$. Assume that $\left(Y_{m}\right)_{m \in \mathbb{N}_{0}}$ is a sequence satisfying the condition (3.15) for some positive numbers $\tau$ and $C$, and such that $Y_{m}$ is summetric for every $m \in \mathbb{N}_{0}$. For $\xi>0$, let $G_{\mathrm{ev}}(\xi)$ be the set defined by (3.11) for $\sigma_{1 ; \boldsymbol{s}}:=\beta_{1 ; \boldsymbol{s}}$ and $\sigma_{2 ; s}:=\beta_{r ; s}$ as in (6.1). Then for the quadrature operator $\mathcal{Q}_{G_{\mathrm{ev}}}(\xi)$ generated by the interpolation operator $\mathcal{I}_{G_{\mathrm{ev}}(\xi)}: \mathcal{L}_{2}\left(W^{r}\right) \rightarrow \mathcal{V}\left(G_{\mathrm{ev}}(\xi)\right)$, we have the following.

(i) For each $n \in \mathbb{N}$ there exists a number $\xi_{n}$ such that $\operatorname{dim} \mathcal{V}\left(G_{\mathrm{ev}}\left(\xi_{n}\right)\right) \leq n$ and

$$
\left\|\int_{\mathbb{I} \infty} u(\boldsymbol{y}) \mathrm{d} \nu_{a, b}(\boldsymbol{y})-\mathcal{Q}_{G_{\mathrm{ev}}\left(\xi_{n}\right)} u\right\|_{V} \leq C n^{-\min (\alpha, \beta)} .
$$

(ii) Let $\phi \in V^{\prime}$ be a bounded linear functional on $V$. For each $n \in \mathbb{N}$ there exists a number $\xi_{n}$ such that $\operatorname{dim} \mathcal{V}\left(G_{\text {ev }}\left(\xi_{n}\right)\right) \leq n$ and

$$
\left|\int_{\mathbb{I} \infty}\langle\phi, u(\boldsymbol{y})\rangle \mathrm{d} \nu_{a, b}(\boldsymbol{y})-\mathcal{Q}_{G_{\mathrm{ev}}\left(\xi_{n}\right)}\langle\phi, u\rangle\right| \leq C n^{-\min (\alpha, \beta)} .
$$

The rate $\alpha$ corresponds to the spatial approximation of a single function in $W^{r}$ as given by (6.2). The rate $\beta$ is given by

$$
\beta:=\left(\frac{2}{q_{1}}-\frac{1}{2}\right) \frac{\alpha}{\alpha+\delta}, \quad \delta:=\frac{2}{q_{1}}-\frac{2}{q_{r}} .
$$

The constants $C$ in (6.6) and (6.7) are independent of $u$ and $n$.

The rate in (6.6) and (6.7) can be improved as $\min \left(\alpha, \frac{2}{q_{1}} \frac{\alpha}{\alpha+\delta}\right)$ if $\left(\left\|u_{\boldsymbol{s}}\right\|_{V}\right)_{\boldsymbol{s} \in \mathbb{F}}$ and $\left(\left\|u_{\boldsymbol{s}}\right\|_{W^{r}}\right)_{\boldsymbol{s} \in \mathbb{F}}$ have $\ell_{p_{1}}$ - and $\ell_{p_{r}}$-summable majorant sequences, respectively, where $1 / p_{1}=1 / q_{1}+1 / 2$ and $1 / p_{r}=1 / q_{r}+1 / 2$, see [34].

Corollary 6.8. Let $a=b$ for the Jacobi probability measure $\nu_{a, b}(\boldsymbol{y})$, and the assumptions of Lemma 6.1 hold for the spaces $W^{1}=V$ with some $0<q_{1}=q<4$. Assume that $\left(Y_{m}\right)_{m \in \mathbb{N}_{0}}$ is a sequence satisfying the condition (3.15) for some positive numbers $\tau$ and $C$, and such that $Y_{m}$ is symmetric for every $m \in \mathbb{N}_{0}$. For $\xi>0$, let $\Lambda_{\mathrm{ev}}(\xi)$ be the set defined by (3.41) for $\sigma_{\boldsymbol{s}}:=\beta_{1 ; \boldsymbol{s}}$ as in (6.1). Then we have the following.

(i) For each $n \in \mathbb{N}$ there exists a number $\xi_{n}$ such that $\left|\Gamma\left(\Lambda_{\mathrm{ev}}\left(\xi_{n}\right)\right)\right| \leq n$ and

$$
\left\|\int_{\mathbb{I}^{\infty}} u(\boldsymbol{y}) \mathrm{d} \nu_{a, b}(\boldsymbol{y})-Q_{\Lambda_{\mathrm{ev}}\left(\xi_{n}\right)} u\right\|_{V} \leq C n^{-(2 / q-1 / 2)} .
$$

(ii) Let $\phi \in V^{\prime}$ be a bounded linear functional on $V$. For each $n \in \mathbb{N}$ there exists a number $\xi_{n}$ such that $\left|\Gamma\left(\Lambda_{\mathrm{ev}}\left(\xi_{n}\right)\right)\right| \leq n$ and

$$
\left|\int_{\mathbb{I} \infty}\langle\phi, u(\boldsymbol{y})\rangle \mathrm{d} \nu_{a, b}(\boldsymbol{y})-Q_{\Lambda_{\mathrm{ev}}\left(\xi_{n}\right)}\langle\phi, u\rangle\right| \leq C n^{-(2 / q-1 / 2)} .
$$

The constants $C$ in (6.8) and (6.9) are independent of $u$ and $n$.

The rate $2 / q-1 / 2$ in (6.8) in Corollary 6.8 improves the rate $2 / q-1 / 2-\varepsilon$ with arbitrary $\varepsilon>0$, which has been obtained in Corollary 3.13 of [33]. 
Acknowledgements. This work is funded by Vietnam National Foundation for Science and Technology Development (NAFOSTED) under Grant No. 102.01-2020.03. It was partially supported by a grant from the Simon Foundation and EPSRC Grant Number EP/R014604/1. The author would like to thank the Isaac Newton Institute for Mathematical Sciences for partial support and hospitality during the programme Approximation, sampling and compression in data science when work on this paper was partially undertaken. He is grateful to the referees for their comments and suggestions which helped him to tremendously improve the presentation of the paper. He expresses special thanks to Christoph Schwab and Nguyen Van Kien for valuable remarks and comments.

\section{REFERENCES}

[1] M. Bachmayr, A. Cohen, D. Dũng and C. Schwab, Fully discrete approximation of parametric and stochastic elliptic PDEs. SIAM J. Numer. Anal. 55 (2017) 2151-2186.

[2] M. Bachmayr, A. Cohen and G. Migliorati, Sparse polynomial approximation of parametric elliptic PDEs. Part I: affine coefficients. ESAIM: M2AN $\mathbf{5 1}$ (2017) 321-339.

[3] M. Bachmayr, A. Cohen, R. DeVore and G. Migliorati, Sparse polynomial approximation of parametric elliptic PDEs. Part II: lognormal coefficients. ESAIM: M2AN 51 (2017) 341-363.

[4] P. Chen, Sparse quadrature for high-dimensional integration with Gaussian measure. ESAIM: M2AN 52 (2018) $631-657$.

[5] A. Chkifa, A. Cohen, R. DeVore and C. Schwab, Sparse adaptive Taylor approximation algorithms for parametric and stochastic elliptic PDEs. ESAIM: M2AN 47 (2013) 253-280.

[6] A. Chkifa, A. Cohen and C. Schwab, High-dimensional adaptive sparse polynomial interpolation and applications to parametric PDEs. Found. Comput. Math. 14 (2014) 601-633.

[7] A. Chkifa, A. Cohen and C. Schwab, Breaking the curse of dimensionality in sparse polynomial approximation of parametric PDEs. J. Math. Pures Appl. 103 (2015) 400-428.

[8] P. Ciarlet, The Finite Element Method for Elliptic Problems. North Holland Publ. (1978).

[9] A. Cohen and R. DeVore, Approximation of high-dimensional parametric PDEs. Acta Numer. 24 (2015) 1-159.

[10] A. Cohen, R. DeVore and C. Schwab, Convergence rates of best $N$-term Galerkin approximations for a class of elliptic sPDEs. Found. Comput. Math. 10 (2010) 615-646.

[11] A. Cohen, R. DeVore and C. Schwab, Analytic regularity and polynomial approximation of parametric and stochastic PDEs. Anal. Appl. 9 (2011) 11-47.

[12] J. Dick, F. Kuo and I. Sloan, High-dimensional integration: the quasi-Monte Carlo way. Acta Numer. 22 (2013) $133-288$.

[13] J. Dick, C. Irrgeher, G. Leobacher and F. Pillichshammer, On the optimal order of integration in Hermite spaces with finite smoothness. SIAM J. Numer. Anal. 56 (2018) 684-707.

[14] D. Dũng, Linear collective collocation and Galerkin approximations for parametric and stochastic elliptic PDEs. Preprint arXiv:1511.03377v5 [math.NA] (2015).

[15] D. Dũng, Galerkin approximation for parametric and stochastic elliptic PDEs. Bull. L.N. Gumilyov Eurasian Nat. Univ. Math. Comput. Sci. Mech. Ser. 1 (2018) 76-89.

[16] D. Dũng, Linear collocation approximation for parametric and stochastic elliptic PDEs. Sb. Math. 210 (2019) $103-227$.

[17] A. Erdélyi, W. Magnus, F. Oberhettinger and F. G. Tricomi, Higher Transcendental Functions, Vol. II. McGraw-Hill (1955).

[18] O. Ernst, B. Sprungk and L. Tamellini, Convergence of sparse collocation for functions of countably many Gaussian random variables - with application to lognormal elliptic diffusion problems. SIAM J. Numer. Anal. 56 (2018) 887-905.

[19] P. Grisvard, Elliptic Problems in Nonsmooth Domains. In: Vol. 24 of Monographs and Studies in Mathematics. Pitman (Advanced Publishing Program), Boston (1985).

[20] M. Gunzburger, C. Webster and G. Zang, Stochastic finite element methods for partial diferential equations with random input data. Acta Numer. 23 (2014) 521-650.

[21] A.-L. Haji-Ali, F. Nobile, L. Tamellini and R. Tempone, Multi-index stochastic collocation convergence rates for random PDEs with parametric regularity. Found. Comput. Math. 16 (2016) 1555-1605.

[22] A.-L. Haji-Ali, F. Nobile, L. Tamellini and R. Tempone, Multi-index stochastic collocation for random PDEs. Comput. Methods Appl. Mech. Eng. 306 (2016) 95-122.

[23] E. Hewitt and K. Stromberg, Real and Abstract Analysis. Springer (1965).

[24] V.H. Hoang and C. Schwab, $N$-term Wiener chaos approximation rate for elliptic PDEs with lognormal Gaussian random inputs. Math. Models Methods Appl. Sci. 24 (2014) 796-826.

[25] D.S. Lubinsky, A survey of weighted polynomial approximation with exponential weights. Surv. Approx. Theory 3 (2007) $1-105$.

[26] D.M. Matjila, Bounds for the weighted Lebesgue functions for Freud weights. J. Approx. Theory 79 (1994) $385-406$.

[27] D.M. Matjila, Convergence of Lagrange interpolation for Freud weights in weighted $L_{p}, 0<p \leq 1$. In: Nonlinear Numerical Methods and Rational Approximation. Kluwer, Dordrecht (1994) 25-35.

[28] F. Nobile, R. Tempone and C.G. Webster, An anisotropic sparse grid stochastic collocation method for elliptic partial differential equations with random input data. SIAM J. Numer. Anal. 46 (2008) 2411-2442.

[29] C. Schwab and C. Gittelson, Sparse tensor discretizations high-dimensional parametric and stochastic PDEs. Acta Numer. 20 (2011) 291-467. 
[30] J. Szabados, Weighted Lagrange and Hermite-Fejér interpolation on the real line. J. Inequal. Appl. 1 (1997) $99-123$.

[31] G. Szegö, Orthogonal Polynomials. In: Vol. 23 of American Mathematical Society Colloquium Publications. American Mathematical Society, Providence, RI (1939).

[32] J. Zech, Sparse-grid approximation of high-dimensional parametric PDEs, Dissertation 25683, ETH Zurich (2018).

[33] J. Zech and C. Schwab, Convergence rates of high dimensional Smolyak quadrature. ESAIM: M2AN 54 (2020) $1259-1307$.

[34] J. Zech, D. Dũng and C. Schwab, Multilevel approximation of parametric and stochastic PDEs. Math. Models Methods Appl. Sci. 29 (2019) 1753-1817. 